
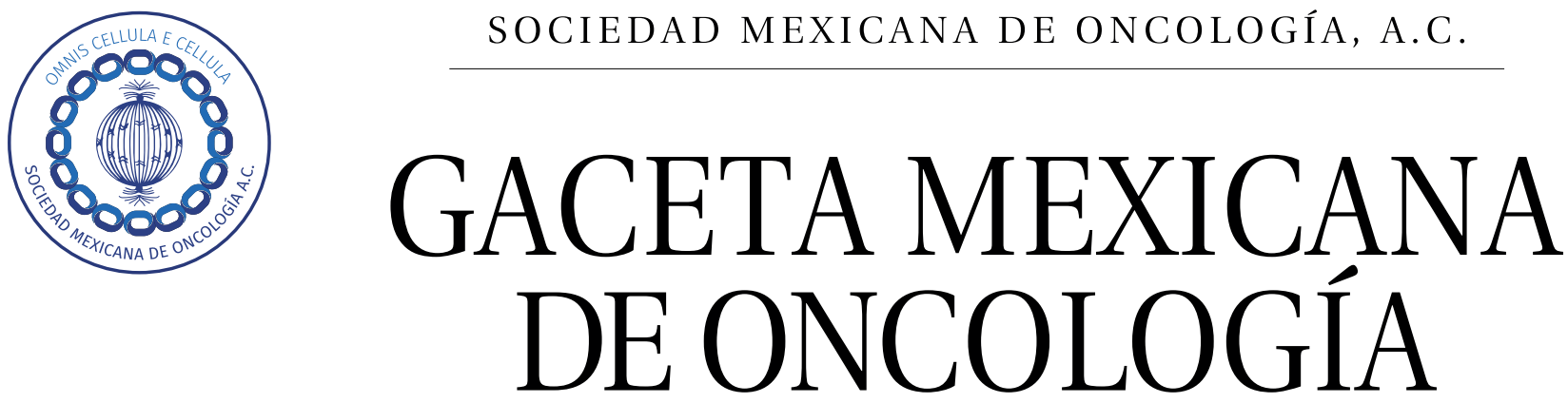

Publicación Oficial de la Sociedad Mexicana de Oncología

\title{
Mexican Consensus on diagnosis and treatment of breast cancer
}

Seventh revision - Colima 2017

\section{Coordinators}

Jesús Cárdenas Sánchez, MD

Medical oncologist

Instituto Estatal de Cancerología, SS

Colima, Col.

Juan Enrique Bargalló Rocha, MD

Surgical oncologist

Instituto Nacional de Cancerología, SS

Ciudad de México

Verónica Bautista Piña, MD

Pathologist

Instituto de Enfermedades de la Mama, FUCAM

Ciudad de México

Guadalupe Cervantes Sánchez, MD

Medical oncologist

Centro Médico Nacional 20 de Noviembre, ISSSTE

Ciudad de México

Aura A. Erazo Valle-Solís, MD

Medical oncologist

Centro Médico Nacional 20 de Noviembre, ISSSTE

Ciudad de México

Christian Haydeé Flores Balcázar, MD

Radiation oncologist

Instituto Nacional de Ciencias Médicas y Nutrición Salvador Zubirán, SS

Ciudad de México
Antonio Maffuz Aziz, MD

Surgical oncologist

Instituto de Enfermedades de la Mama, FUCAM

Ciudad de México

Víctor Manuel Pérez Sánchez, MD

Pathologist

Instituto Nacional de Cancerología, SS

Ciudad de México

Adela Poitevin Chacón, MD

Radiation oncologist

Médica Sur

Ciudad de México

Efraín Salas González, MD

Medical oncologist

Centro Médico de Occidente, IMSS

Guadalajara, Jal.

Laura Torrecillas Torres, MD

Medical oncologist

Centro Médico Nacional 20 de Noviembre, ISSSTE

Ciudad de México

Vicente Valero Castillo, MD

Medical oncologist

M.D. Anderson Cancer Center

Houston, TX, United States 


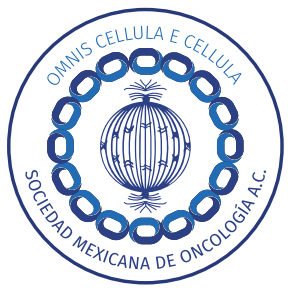

SOCIEDAD MEXICANA DE ONCOLOGÍA, A.C.

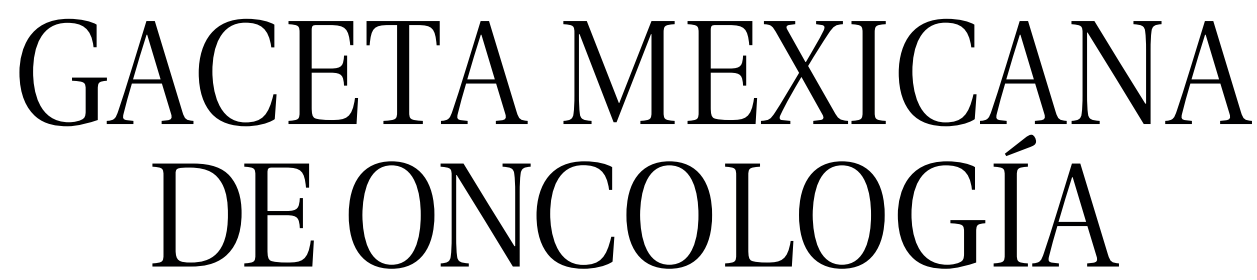

Publicación Oficial de la Sociedad Mexicana de Oncología

\section{Index}

I. Introduction $\ldots \ldots \ldots \ldots \ldots \ldots \ldots \ldots \ldots \ldots \ldots \ldots \ldots \ldots \ldots \ldots \ldots \ldots \ldots \ldots$

II. Epidemiology of breast cancer in Mexico $\ldots \ldots \ldots \ldots \ldots \ldots \ldots \ldots \ldots \ldots \ldots \ldots \ldots \ldots \ldots \ldots \ldots$

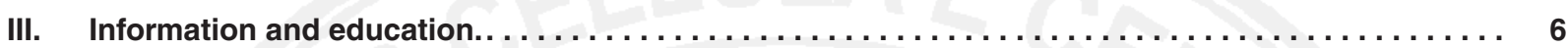

IV. Breast cancer primary prevention $\ldots \ldots \ldots \ldots \ldots \ldots \ldots \ldots \ldots \ldots \ldots \ldots \ldots \ldots \ldots \ldots \ldots \ldots$

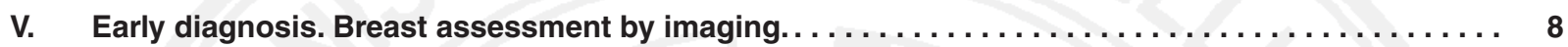

Screening studies

Imaging studies
1. Mammography
2. Breast ultrasound (US)
3. Magnetic resonance imaging (MRI)
4. Positron emission tomography (PET)

VI. Non-palpable suspicious lesion

- Biopsy indication: lesions categorized as BI-RADS 4 and 5
A. Aspiration biopsy (cytology)
B. Core needle and vacuum-assisted core biopsy (histology)

VII. Histopathological study

1. Recommendations for conservative surgery specimen handling and report

2. Recommendations for infiltrating breast carcinoma histopathological report

3. Recommendations for post-treatment specimens report

4. High grade precursor lesions and breast carcinoma in situ histopathological report

5. Recommendations for sentinel lymph node histopathological report

6. Recommendations for breast tumor fine needle aspiration biopsy (FNAB) report

7. Recommendations for the report of axillary lymph node FNAB with possible metastasis

8. Recommendations for the report of prognostic-predictive factors by immunochemistry

9. Recommendations for molecular biology

10. Invasive breast carcinoma molecular signatures

VIII. TNM classification

IX. Carcinoma in situ. . . . . . . . . . . . . . . . . . . . . . . . . . . . . .

- Ductal carcinoma in situ (DCIS)

- Lobular carcinoma in situ (LCIS)

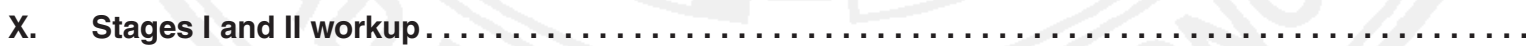

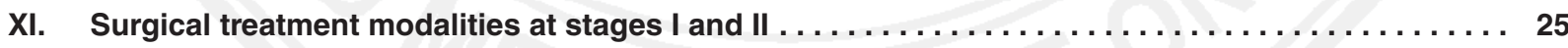
A. Conservative treatment
B. Radical treatment (mastectomy)
C. Oncoplastic surgery
D. Surgical treatment of axilla
E. Breast reconstruction
F. Risk-reducing mastectomy 

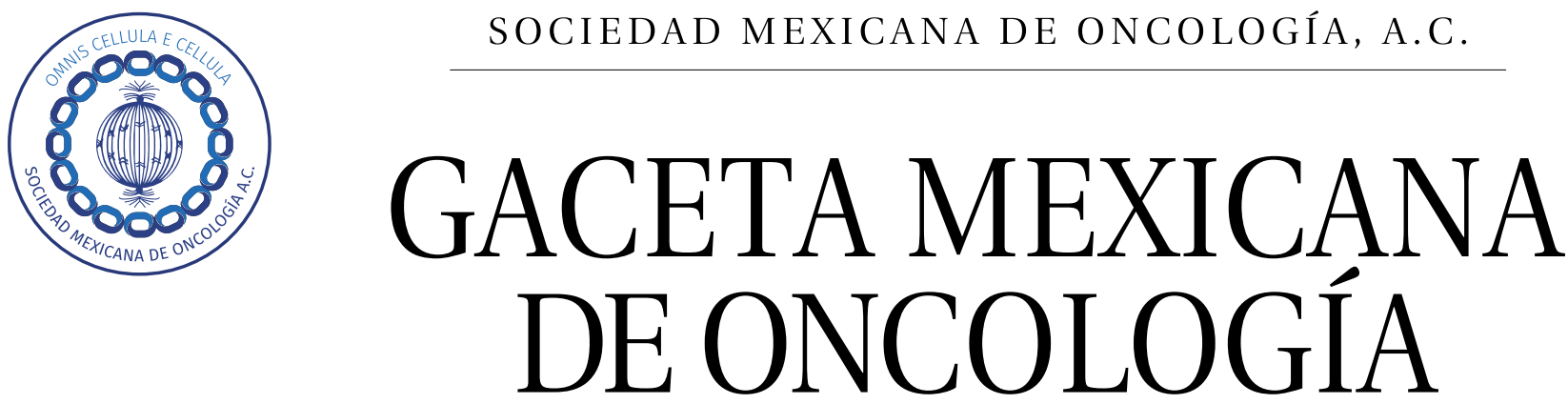

Publicación Oficial de la Sociedad Mexicana de Oncología

XII. Adjuvant systemic therapy at operable stages . . . . . . . . . . . . . . . . . . . .

Definition, indications and objectives

Selection of adjuvant systemic therapy
A. Adjuvant treatment with chemotherapy
B. Adjuvant treatment with hormone therapy
C. Adjuvant treatment with targeted therapy

Breast cancer medical treatment-derived mid and long-term toxicity
A. Cardiotoxicity
B. Leukemia and myelodysplastic syndrome
C. Neuropathy
D. Fatigue
E. Cognitive dysfunction
F. Medical treatment-induced menopausal symptoms
G. Chemotherapy-induced ovarian failure

XIII. Adjuvant radiotherapy .

- Post-operative radiotherapy in conservative management

- Indications for adjuvant radiotherapy to lymph node chains

- Radiotherapy to the chest wall after mastectomy

- Radiotherapy associated with chemotherapy, targeted therapies and hormone therapy

XIV. Neoadjuvant treatment of stage II and III breast cancer, including locally advanced disease . . . . .

Neoadjuvant treatment in patients with operable and inoperable stages

- Neoadjuvant chemotherapy

- Targeted therapies in neoadjuvancy

- Neoadjuvant hormone therapy

- Response assessment during neoadjuvant treatment

- Post-neoadjuvancy treatment

- Inflammatory breast cancer

- Surgery in locally-advanced disease

- Radiotherapy aspects

- Radiotherapy-induced toxicity

XV. Metastatic breast cancer treatment Introduction

Treatment according to breast cancer subtype

A. Metastatic breast cancer with positive hormone receptors and negative HER-2 neu

B. Hormone receptor-positive, HER-2 neu-positive metastatic breast cancer

C. Triple-negative metastatic breast cancer or with positive hormone receptors, negative HER-2 neu not candidate to hormone therapy

D. HER-2 neu-positive metastatic breast cancer

The role of surgery in metastatic disease
A. Resection in metastatic disease
B. Primary tumor resection in metastatic disease
C. Palliative primary tumor resection in metastatic disease 

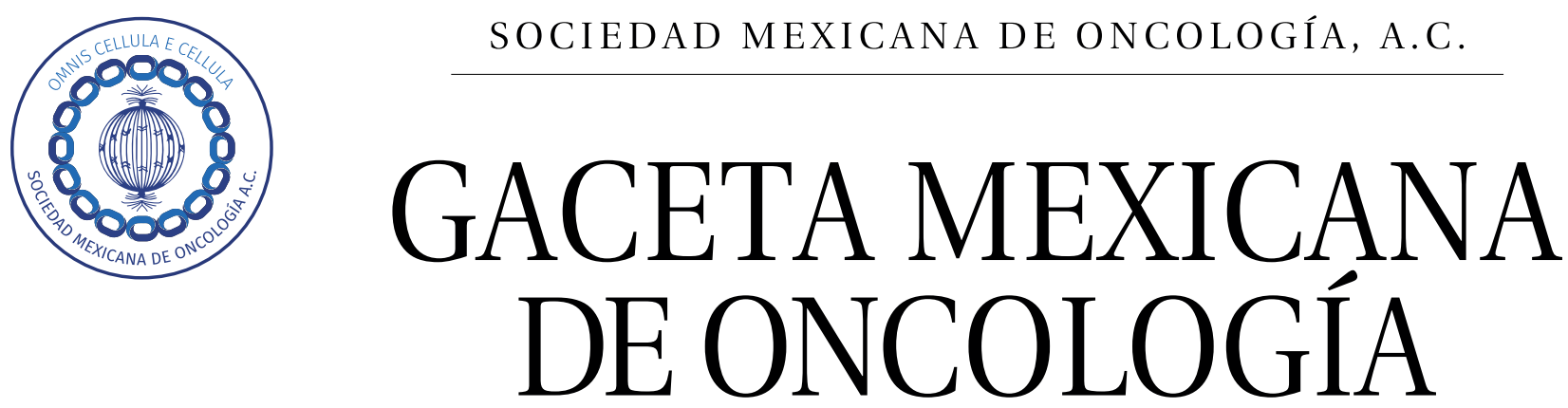

Publicación Oficial de la Sociedad Mexicana de Oncología

The role of radiotherapy in metastatic disease

- Bone metastasis

- Brain metastasis

- SBRT in oligometastatic disease

Bisphosphonates and receptor-activator NF-KB ligand (RANKL) inhibitors in bone metastases, adjuvancy and with aromatase inhibitors

XVI. Breast cancer in young women $\ldots \ldots \ldots \ldots \ldots \ldots \ldots \ldots \ldots \ldots \ldots \ldots \ldots \ldots$

XVII. Treatment in advanced age patients . . . . . . . . . . . . . . . . . . . . . . 46

XVIII. Male breast cancer. . . . . . . . . . . . . . . . . . . . . . . . . . . . . . . 47

XIX. Breast cancer associated with pregnancy and breastfeeding. . . . . . . . . . . . . 48

- General guidelines

- Surgery

- Radiotherapy

- Systemic treatment

- Prognosis

XX. Follow-up after curative intent treatment. . . . . . . . . . . . . . . . . . . 49

XXI. Hormone replacement therapy . . . . . . . . . . . . . . . . . . . . . . . . . . 49

XXII. Genetics and breast cancer . . . . . . . . . . . . . . . . . . . . . . . . . . . 49

XXIII. Psycho-oncologic aspects in breast cancer . . . . . . . . . . . . . . . . . . . 52

XXIV. Physical rehabilitation for the breast cancer patient $\ldots \ldots \ldots \ldots \ldots \ldots \ldots \ldots$

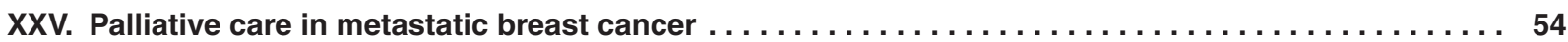

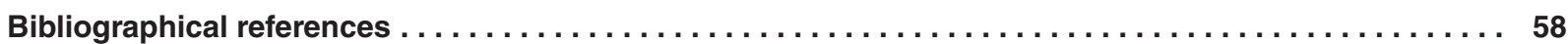

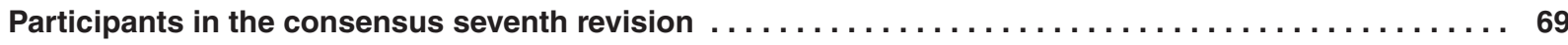




\section{Mexican Consensus on diagnosis and treatment of breast cancer}

\section{Introduction}

The first National Consensus on Diagnosis and Treatment of Breast Cancer was carried out in Colima in 1994; its conclusions were widely diffused ${ }^{1}$ and have been useful as a guide for oncologists and other physicians of related specialties. Since then, periodical review meetings have taken place, where available knowledge and information have been updated, and participation was extended to other subspecialties and disciplines related to the diagnosis and treatment of this disease. The conclusions were published in specialized journals ${ }^{2-7}$ and are available online on the Consensus page (www.consensocancermamario.com) and other institutions and oncology societies' websites.

Since these publications have been widely diffused and constantly updated, practically all oncologists of the country are aware of the Consensus conclusions and use them as a tool to support decision-making in their daily oncologic practice. In addition, they are part of several oncologic institutions guidelines and of the documentation the Mexican Official Standard on the subject is based on. ${ }^{8}$

On this occasion, we met in Colima again, on January 2017, with the purpose to review recent advances in the field of breast cancer prevention, diagnosis and treatment. Nearly 100 nation-wide renowned physicians from all institutions and specialties related to this disease were called together and, in working groups, they analyzed the updated information of each area with the purpose to present it at plenary session for approval. This time, the subject of the negative impact of diagnostic and treatment initiation delay was added, stressing on the responsibility the health system has in these problems.

We hope that the conclusions of this seventh revision herein presented serve as a guide for the medical community in general and for oncologists in particular, in order for them to offer patients with this disease an accurate diagnosis, as well as an optimal and updated treatment.

\section{Epidemiology of breast cancer in Mexico}

Worldwide, breast cancer is the most frequent tumor and the most common cause of death in women that die from malignances. ${ }^{1}$ Nearly 1.67 million women are estimated to be diagnosed with breast cancer every year and 552,000 patients die from this disease.' Control and survival vary according to the population and region where this neoplasm occurs. In poor and developing countries, 5 -year survival is $30 \%$ to $45 \%$, in contrast with fully developed countries, where it is $80 \%$. These results depend heavily on access to cancer opportune detection (COD) and optimal treatment. ${ }^{2}$

On the other hand, incidence rates vary considerably between regions and countries of the world. There is a large number of epidemiological studies pointing at possible causes of this diversity in breast cancer pattern of occurrence. ${ }^{3}$ Among these, reproductive factors such as age at menarche, menopause and first delivery stand out, as well as breastfeeding time; exposure to hormone replacement therapy, postmenopausalobesity, alcohol intake and access to COD are also important. In addition, BRCA1 and BRCA2 genes' mutation represents an important risk factor for the development of this disease; however, its impact on the general population is lower. ${ }^{4}$

In fully developed countries, breast cancer mortality has consistently decreased; such is the case of the United States, Denmark and the United Kingdom, among others. ${ }^{5}$ This reduction has been associated with optimal treatment and efficient, opportune 


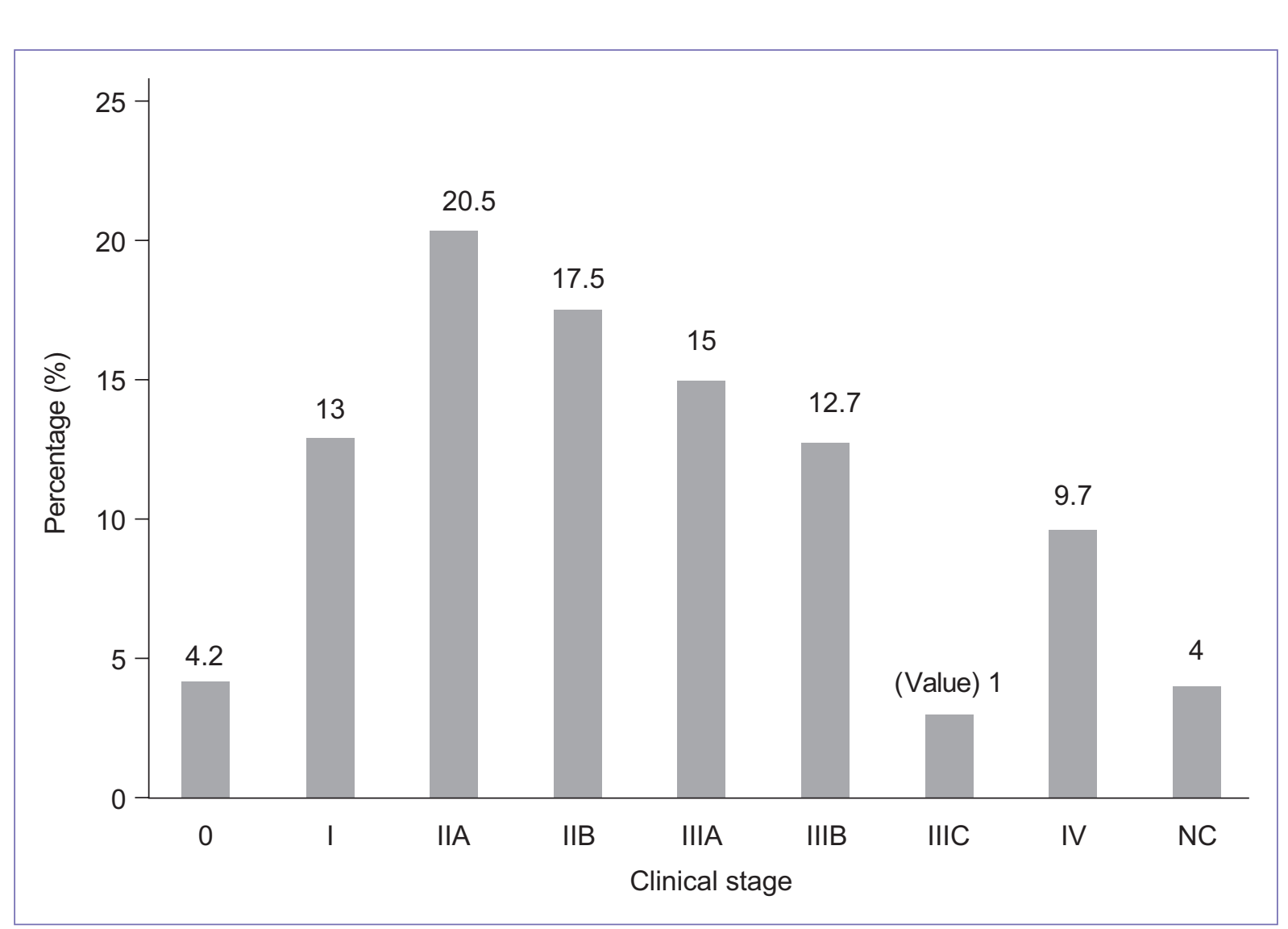

Figure 1. Distribution by clinical stage ( $n=10,433$ ).

detection. In Mexico, breast cancer has shown an increase both in incidence rates and mortality, with the latter being associated with late diagnosis and COD program poor efficacy.

The International Agency for Research on Cancer (IARC) last estimates indicate that, in 2013, in our country there were 23,687 new cases and 5,902 patients died. $^{5}$

As of 2007, the Seguro Popular (SP) (people's health insurance) incorporated breast cancer to the program known as catastrophic expenses, which warrants free care of the disease with optimal treatment at all stages. In a sample of more than 10 thousand cases with diagnosis and treatment in the SP setting attended to at the INCan and the FUCAM (Mexico City), as well as at the ISSEMyM (Toluca), ${ }^{6}$ mean age at diagnosis was identified to be 52.5 years and $32 \%$ of patients were found to have comorbidities (high blood pressure $21 \%$, diabetes $12.1 \%$ and active smoking $4 \%$ ). Tumor size in this population had a median of $3 \mathrm{~cm}$ at diagnosis.

With regard to immunophenotype, $65 \%$ was hormone receptor-positive, $21 \%$ was HER-2 neu-positive and
$16 \%$ was triple-negative. Figure 1 shows that, at initial diagnosis, $58 \%$ of patients were at advanced stages of the disease (Illb or more advanced). ${ }^{6}$ It is important mentioning that this percentage remained practically unchanged from 2007 to 2015 (period of this analysis), and it is therefore concluded that although universal access to treatment is efficient, we have not yet been able to improve early detection, which represents a historical public health pending issue for better control of this disease.

\section{Information and education}

Sustainable development global goals proposed by the World Health Organization (WHO) point at ensuring a healthy life by promoting wellbeing for all people at all ages and with gender equality, which requires for the community-based education component to be reinforced. In the specific subject of breast cancer, community-based actions directed to adult people have been established to precisely impact on early detection. ${ }^{1}$ 
Prevention activities include educational communication to the population for awareness on risk factors and promotion of healthy lifestyles, since physical activity, nutritional components and body mass index modify the possibility of sporadically developing breast cancer. The WHO also recommends for education on breast cancer to be aimed at sensitizing women on the importance of knowing their breasts' normal characteristics and seeking opportune medical care if they discover any abnormality. ${ }^{2}$

The differences on medical care of this pathology and their consequences and impact on health are reflected on statistics about life years lost due to premature death and quality of life loss due to disability, and the urgency for specific and systematized actions for detection, early diagnosis and opportune referral is therefore vital for those who suffer from this condition. ${ }^{3}$

Known risk factors for the development of breast cancer are:4

\section{A Biological}

- Female gender.

- Aging: the older the age, the higher the risk.

- Personal or family history of breast cancer in mother, daughters or sisters.

- Prior findings of atypical ductal hyperplasia, radial or stellate image, as well as lobular carcinoma in situ by biopsy.

- Menstrual life longer than 40 years (menarche prior to 12 years and menopause after 52 years of age).

- Breast density.

- Being carrier of the BRCA1 or BRCA2 genes.

$B$ latrogenic or environmental:

- Exposure to ionizing radiation, especially during development or growth (in utero, at adolescence).

- Radiotherapy to the chest.

C Risk factors related to reproductive history:

- Nulligravida.

- First full-term pregnancy after 30 years of age.

- Hormone therapy at perimenopause or postmenopause for more than 5 years.

$D$ Risk factors related to lifestyle:

- Carbohydrate-rich and fiber-low nutrition.

- Diet rich both in animal fat and trans fatty acids.

- Obesity. ${ }^{5-7}$

- Sedentary lifestyle.

- Alcohol consumption of more than $15 \mathrm{~g} /$ day.

- Smoking.

The most important lifestyle-related risk factor is obesity and, given that in our country this condition is present in a highly elevated percentage of the population, it represents a serious public-health problem.
Obesity and breast cancer are two currently extremely prevalent conditions and with high impact on society.

Obese women are at higher risk for the development of breast cancer after menopause in comparison with non-obese women. This appears to be explained by higher levels of circulating estrogen. In addition, women with a prior history of breast cancer who develop obesity are at higher risk for tumor relapse or a second primary lesion.

There are reports indicating that a waist circumference larger than $80 \mathrm{~cm}$ considerably increases the risk for breast cancer; on the other hand, menarche at an early age associated with states of morbid obesity is another important factor in early genesis of this disease.

Care of the obese patient should include a vegetable-based diet, promotion of physical activity, components of behavioral change and long-term follow-up.

On the other hand, scientific bibliography supports that physical exercise is an effective activity to reduce the risk for suffering from breast cancer. ${ }^{8-11}$

General recommendations for physical activity are the following

- 150 minutes/week of moderate-intensity aerobic exercise, walking or bicycle riding.

- 75 minutes/week of vigorous-intensity aerobic activity, or else, running, jogging, jumping rope, swimming, playing basketball, etc. (www.cdc.gov/healthyweight/ physical_activity/index.html).

Motivation in women is essential to achieve treatment adherence and to maintain its effects on the long-term. Inclusion of physical activities in the community helps to prevent chronic diseases in general, and such activities are protective against breast cancer and, for this reason, their importance should be diffused by mass media to the entire population.

\section{Breast cancer primary prevention}

\section{Risk-reducing therapy}

The criteria applied in studies to consider high-risk women as candidates to chemoprevention include: ${ }^{1}$

- Age > 60 years.

-35 to 59 years of age with 5 -year risk $\geq 1.66 \%$ in the Gail model for breast cancer.

- Age $\geq 35$ years with previous history of lobular or ductal carcinoma in situ, ductal or lobular atypical hyperplasia. 
- BRCA-1 or BRCA-2 mutation carriers without prophylactic mastectomy. ${ }^{2}$

Pharmacological intervention:

In women at high risk, ${ }^{1,2}$ the use of the following agents is recommended:

a)Tamoxifen at a dosage of $20 \mathrm{mg} /$ day in pre- and postmenopausal women or raloxifen at a $60 \mathrm{mg} /$ day dosage in postmenopausal women for a period of 5 years, based on the P-1 (NSABP), RUTH 4, MORE 4, CORE, STAR 2 and IBIS-I trials. Their use was shown to reduce the risk for invasive ductal carcinoma and were approved for this purpose.,3-10 There are no randomized trials with patients younger than 35 years.

b)Aromatase inhibitors (AI) in postmenopausal women:

- Exemestane (MAP-33 trial) and anastrozole (IBISII6) were shown to reduce the risk for invasive breast cancer. ${ }^{11,12}$ These agents have not yet been approved by regulatory agencies for this indication.

To decide on the use of risk-reducing drugs, other factors that might contraindicate them have to be taken into account; in the case of tamoxifen, previous history of thromboembolic or atypical endometrial hyperplasia events, and for aromatase inhibitors, significant osteopenia or osteoporosis.

In pre- and postmenopausal women, recommendations with regard to modifiable risk factors should be made:

- Prevent or decrease obesity.

- Practice physical activity.

- Limit alcohol consumption.

- Avoid smoking.

\section{Early diagnosis. Breast assessment by imaging}

\section{Screening studies}

\section{General recommendations}

- Monthly breast self-exam from 18 years of age on (7 days after menstruation conclusion).

- Annual clinical breast examination from 25 years of age on.

- Annual screening mammography in asymptomatic women from 40 years of age on.

- Breast US is the initial study of choice in women younger than 35 years with breast pathology.

\section{Imaging studies}

The use of imaging studies such as mammography, ultrasound (US), magnetic resonance imaging (MRI) and, more recently, molecular studies, allows to detect, characterize and evaluate the disease and its extent, as well as for breast lesions evolution follow-up.

Histopathological analysis is the gold standard for diagnosis; percutaneous biopsies with core needle and X-ray or ultrasound-guided vacuum-assisted systems are the method of choice for non-palpable lesions suspected of malignancy and, recently, also accepted for palpable lesions.

\section{Mammography}

Mammography was regarded for many years as the only imaging method to show a $15 \%$ to $20 \%$ mortality decrease in 40 to 74 -year-old women owing to the opportunity of early diagnosis. ${ }^{1}$

Recent randomized, controlled studies demonstrate that the use of screening mammography does not decrease, at least significantly, the number of breast cancer deaths; ${ }^{2}$ however, it is documented that it improves patient overall survival and increases life span 3

It is also important to consider that screening mammography may cause overdiagnosis and unnecessary treatments $(20 \%)$, anxiety in women ${ }^{3,4}$ and radiation-induced cancer (one in thousand screened women). ${ }^{5}$

\section{Mammography}

- Conventional acquisition. The mammography device is analogue and image acquisition is performed with the screen-film system, which additionally requires automatic development equipment.

- Digital acquisition. Through detectors integrated to the mammography device itself (digital) or external detectors (digitalized, CR); the study is printed with a high-resolution laser equipment.

Digital mammography

- It uses a digital detector. Image acquisition, processing and visualization are managed independently, which represents higher advantage with regard to the analogue system; in addition, the percentage of repetitions owing to constant image quality control is reduced, which results in higher productivity and lower ionizing radiation dose.

- From the clinical point of view, digital mammography increases breast cancer detection in patients with dense breasts, which are a recognized risk factor for breast cancer. ${ }^{6}$ 
Table 1.

\begin{tabular}{|c|c|c|c|}
\hline Category & & Recommendations & \\
\hline 0 & $\begin{array}{l}\text { Insufficient for diagnosis. There is } 13 \% \\
\text { possibility of malignancy }\end{array}$ & $\begin{array}{l}\text { Need evaluation with additional mammographic images or other } \\
\text { studies (ultrasound and magnetic resonance), as well as compariso } \\
\text { prior studies }\end{array}$ & $\frac{\infty}{\frac{-}{2}}$ \\
\hline 1 & $\begin{array}{l}\text { Negative } \\
\text { No findings to report }\end{array}$ & Annual mammography in women from 40 years of age on. & है \\
\hline 2 & Benign findings & Annual mammography in women from 40 years of age on. & 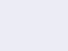 \\
\hline 3 & $\begin{array}{l}\text { Probably benign findings. Less than } 2 \% \\
\text { probability of malignancy }\end{array}$ & $\begin{array}{l}\text { Requires unilateral imaging follow-up of the side with suspicious fin } \\
\text { every six months for } 2 \text { or } 3 \text { years }\end{array}$ & nding \\
\hline 4 & $\begin{array}{l}\text { Findings suspicious of malignancy. It is } \\
\text { subdivided into: } \\
4 a-\text { Low suspicion for malignancy } \\
4 b-\text { Moderate suspicion for malignancy } \\
4 c-\text { Moderate findings of suspicion for } \\
\text { malignancy, but not classical }\end{array}$ & Requires biopsy. & 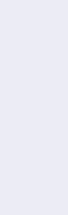 \\
\hline 5 & Classically malignant & Requires biopsy. & $\frac{0}{0}$ \\
\hline 6 & With histological diagnosis of malignancy & Awaiting definitive treatment or treatment response evaluation. & $\bar{\varepsilon}$ \\
\hline
\end{tabular}

Digital mammography has the capability for advanced applications such as:

- Telemammography.

- CAD: computer-assisted detection system. It was created as a second reader to aid in interpretation by identifying regions with grouped microcalcifications and masses. In general, it increases sensitivity but decreases specificity. ${ }^{7}$

- Tomosynthesis or three-dimensional mammography.

- Multiple mammographic images are obtained from different angles.

- 2D (two-dimensional) and 3D (three-dimensional) mammography, which is superior to $2 \mathrm{D}$ alone for detection, although it doubles the radiation dose, it improves breast cancer detection and reduces the number of repetitions and call-backs (10\%).

- Synthesized mammography involves obtaining 3D images based on a two-dimensional reconstruction, which reduces the radiation dose and also highlights areas of architectural distortion, masses and microcalcifications. ${ }^{8}$

Regardless of the mammographic technique used, there has to be a quality assurance program involving the physical area, the equipment, the personnel, study interpretation and patient reference.

The mammography should be interpreted and the conclusion expressed using the BI-RADS system (Breast Imaging Reporting and Data Systems, American College of Radiology, Mammography, $5^{\text {th }}$ ed., 2013) (Table 1).

\section{Diagnostic mammography}

It is performed in case of a mammography with any detected anomaly and in the following situations:

- Dense breast.

- Breast lesions detected with other imaging modality and that clinically require this study.

- Palpable mass or tumor.

- Blood-stained secretion through the nipple.

- Changes in nipple skin or areola.

Special indications for mammography

- Young woman with clinical suspicion of breast cancer, regardless of age.

- History of familial breast cancer at early ages. Annual mammography will be indicated from 30 years of age on, or 10 years before the age of the youngest relative with cancer (not prior to 25 years of age). Magnetic resonance imaging should be considered as a complement in this risk group.

- Prior breast biopsy with histological report consistent with high-risk lesions.

\section{Breast ultrasound (US)}

This is a valuable tool, complementary to diagnostic mammography. It requires high-resolution devices, in addition to experience and knowledge on the anatomy and pathology of the mammary gland and its ulltrasonographic assessment. US should be performed with a high-frequency, broadband and variable focal zone (ideally between 12 and $18 \mathrm{MHz}$ ) linear transducer. ${ }^{9}$ 
Targeted ultrasound is the complement of diagnostic mammography owing to its usefulness to difference cystic from solid nodules, and out of these, benign from malignant. Malignant findings in solid nodules include spiculations, angular margins, marked hyperechogenicity, sonographic posterior acoustic shadowing, microcalcification, duct extension, branching pattern, 1 to $2 \mathrm{~mm}$ microlobulations, deeper (taller) than wide, skin thickening and Cooper's ligaments, which were described by doctor Stavros since 1995.

Screening US is indicated in patients with dense breasts and negative mammography. Numerous studies have confirmed that, in these cases, ultrasound demonstrates from two to five additional occult carcinomas per 1000 women. ${ }^{10}$ Usually, these occult carcinomas in mammography and detected by US are invasive and lymph node-negative.

Screening ultrasound should be considered also in patients at high risk for breast cancer who do not tolerate undergoing MRI.

Clinical indications for breast ultrasound

- Assessment of palpable anomalies

- Assessment of mammography and magnetic resonance imaging-detected anomalies.

- Evaluation of breast implants.

- Guide for interventional procedures

- Radiotherapy treatment planning

- Assessment of axillary lymph nodes

Breast ultrasound improved-vision modality is useful to measure large-size lesions, to assess multifocality (lesions in the same quadrant with less than $5-\mathrm{cm}$ distance in between within the same ductal system) and/ or multicentric disease (lesions located at different quadrants or with more than $5-\mathrm{cm}$ in between within different ductal systems). ${ }^{11}$

Color Doppler and power Doppler are useful to assess cysts and complex cystic masses with solid component; if vascularity is demonstrated within a simple or complex cyst, or complex mass, it confirms the presence of a solid component that requires biopsy.

Elastography improves ultrasonographic evaluation specificity of lesions classified as BI-RADS 4 and 4A, including complex cysts.

In the study by Moon et al. of 201 patients newly diagnosed with breast carcinoma, staging ultrasound demonstrated multi-focal or multicentric disease in $14 \%$ of patients and contralateral disease in $4 \%$, which led to treatment change in $28 \%$ of them. ${ }^{12}$

Some MRI-detected lesions are mammographically occult, but can be found by means of targeted ultrasound (second focused examination). Recent studies have demonstrated a detection increase from $46 \%$ to $71 \% .^{13}$

Interval cancer is 18 -fold more common in women with dense breasts and the prognosis is worse than in screening-detected malignancies.

Various studies have demonstrated that, in women with dense breast tissue, screening ultrasound can detect mammographically occult carcinomas ( 3 to 4.2 additional carcinomas for every 1000 examined women). ${ }^{13}$ Breast density is an important factor in breast carcinoma diagnosis, since it reduces mammographic sensitivity for detection; in addition, it entails a significant increase in the risk for developing this pathology (4.7-fold higher than in women with fatty breasts).

The role of the radiologist in breast cancer staging is to demonstrate, prior to a surgical procedure, the presence of axillary metastases with a positive predictive values that is sufficiently high to allow the surgeon to decide when to carry out axillary dissection. ${ }^{14}$ The presence of axillary metastases and the size of the primary tumor are two prognostic factors to evaluate patients with invasive breast cancer and they determine the use of systemic chemotherapy and radiotherapy. $\mathrm{T} 1$ and $\mathrm{T} 2$ lymph node-negative patients undergo sentinel lymph node procedure. ${ }^{15}$

Ultrasound is the basic tool for axillary lymph node assessment; it has a moderate sensitivity, but it can be highly specific, especially when morphological criteria are affected. A normal axillary lymph node should be oval, with well-defined borders and discretely hypoechoic and uniform cortex. The echogenic hilum should constitute most part of the lymph node, and vascularity can be demonstrated with color Doppler.

Findings such as fatty hilum loss and vascularity outside the hilum are more important criteria than lymph node size to identify metastasis. Focal or diffuse cortical thickening is considered to be the earliest sign to identify metastasis, but it is a criterion that is difficult to apply and that has a low predictive value because it is not specific. It can be subjectively or specifically assessed by measuring the cortex thickness, which should be thinner than 2 to $3 \mathrm{~mm}$.

Tumor invasion can modify lymph node morphology and completely replace it, which may interfere in radiotracer or staining uptake when a dye is used in the sentinel lymph node procedure, since these compounds cannot penetrate when it is invaded and obstructed..$^{15}$

Lymph nodes with suspicious morphology on imaging undergo FNAB or core needle biopsy to avoid anesthetic risk, surgical time and higher cost. FNAB 
has a reported diagnostic sensitivity of $25 \%$ to $87 \%$; core needle biopsy, $90 \%$ to $94 \% .^{16}$

\section{Magnetic resonance imaging (MRI)}

$\mathrm{MRI}$ is an imaging method that is complementary to mammography and breast US in breast cancer diagnosis, staging and follow-up, as well as in the detection of this condition in high-risk women. This method does not use ionizing radiation and provides not only morphological but also functional information by means of endovenous injection of a paramagnetic substance (gadolinium) $;{ }^{17}$ it requires a scanner of at least 1.5 tesla and special antenna for the mammary gland.

Multiple sequences and perfusion curve (dynamical) are used to obtain the images.

Type IA curve has a slow rise and represents benign pathology in $100 \%$ of cases; type IB curve is a variant of the previous one where $94 \%$ of lesions are benign. Type II curve or plateau is of indeterminate type and is associated with malignancy in more than half the cases (64\%). Type III curve has a rapid rise and immediate washout, and is present in most breast cancers (87\%). ${ }^{18}$

MRI has a higher number of false negatives in tumors smaller than $3 \mathrm{~mm}$, as well as in low grade carcinoma in situ and lobular carcinoma and, therefore, for an accurate diagnosis, integration of morphological and functional characteristics together with mammographic and US findings is fundamental.

The conclusion and recommendations should be expressed using the BI-RADS system. ${ }^{19}$

Specificity of this method is increased with the spectroscopic technique (virtual biopsy), which allows the quantification of choline, a cell-proliferation tissue marker that provides biochemical information of tissues. Another technique is diffusion, which is based on the movement of water molecules within the tissue and is useful in the differentiation of benign and malignant lesions. ${ }^{20}$

Indications for contrasted magnetic resonance imaging

- Assessment of margins after primary tumor excision, local recurrence, treatment response, search for occult primary tumor with axillary metastases, pregnancy and suspected breast cancer, screening in patients with high risk and dense breasts, alternating with mammography and US; guide for biopsies of lesions only visible with this method and not corroborated in a US focused second examination.

- Preoperative use of MRI to evaluate disease extent is not recommended because it has not shown to improve overall survival or to decrease re-excision rates or reduce costs. ${ }^{21}$

- Non-contrasted MRI is indicated in breast implants integrity evaluation, particularly with suspected intracapsular rupture or other complications.

\section{Positron-emission tomography (PET)}

PET is diagnostic imaging that combines computerized tomography (CT) with nuclear medicine and simultaneously enables not only morphological, but also molecular (metabolic) examination, with precise localization of a metastatic lesion, after endovenous injection of a radiotracer, usually 18-fluorodeoxyglucose (FDG).

$\mathrm{PET} / \mathrm{CT}$ is an alternative in the detection of locoregional recurrence and distant metastasis, treatment response assessment and follow-up.

\section{Non-palpable suspicious lesion ${ }^{1}$}

Until a few years ago, excisional biopsy, after marking with percutaneous needle, was the only diagnostic tool in clinically non-palpable lesions. Currently, core needle (trucut) biopsy has become a diagnostic evaluation tool in breast non-palpable lesions that avoids excisional biopsies in benign cases, brings down costs and reduces risks for the patient, with minimal change of breast tissue that may alter follow-up in subsequent mammographies. ${ }^{2}$

In the cases of malignant neoplasms, it enables the surgeon to plan treatment alternatives together with the patient. A guiding method should be selected whereby the lesion is best visualized (microcalcifications with X-ray, masses or nodules with US and MRI).

Image-guided biopsy improves diagnostic accuracy, including cases of palpable tumor.

Biopsy indication: lesions categorized as BI-RADS 4 and 5

1. Tumor or mass.

2. Microcalcifications.

3. Breast density asymmetry.

4. New density or changes in already detected density in mammographic follow-up.

5. Architectural distortion.

\section{A. Aspiration biopsy (cytology)}

Cytology by fine needle aspiration has important limitations; it requires great experience from both the radiologist who performs it and the cytologist who interprets it. 
Table 1.

\begin{tabular}{|c|c|c|c|}
\hline Type of biopsy & Type of lesion & Needle caliber & \\
\hline FNAB & $\begin{array}{l}\text { Cysts, axillary lymph nodes } \\
\text { Not recommended in breast primary tumor }\end{array}$ & $22-25 \mathrm{G}$ & $\stackrel{\infty}{\circ}$ \\
\hline Core needle & Solid lesions & \multicolumn{2}{|c|}{11 and $14 \mathrm{G}$ are the most widely used } \\
\hline $\begin{array}{l}\text { Automated vacuum-assisted core biopsy, } \\
\text { Mammotome Vacora, Suros, etc. }\end{array}$ & Main application in biopsy of calcifications & $\begin{array}{l}9,11 \text { and } 14 \mathrm{G} \\
\text { Skin incision, } 5 \text { to } 8 \text { specimens } \\
\text { required }\end{array}$ & 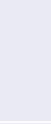 \\
\hline Surgical biopsy & $\begin{array}{l}\text { Lesions that cannot be percutaneously } \\
\text { biopsied (technical limitation), presence of } \\
\text { multiple lesions } \\
\text { Discordant previous core needle biopsies }\end{array}$ & & $\frac{\frac{\dot{\omega}}{\underline{\underline{U}}}}{\underline{\underline{\Omega}}}$ \\
\hline
\end{tabular}

However, it is important for the result usefulness to be considered for decision making in a multidisciplinary approach, since it is a procedure that may help in the assessment of lymph nodes with suspicious morphology.

- It has a sensitivity that ranges from $27 \%$ to up to $100 \%$, specificity of $95 \%$ to $100 \%$, false negative (FN) index of $3.7 \%$ to $19 \%$ and false positive index of $0.68 \%$. Its positive predictive value (PPV) is $64 \%$ to $100 \%$ and negative predictive value (NPV), $59 \%$ to $80 \%{ }^{3}$

- Marking of lymph nodes cytologically reported as metastatic provides better pathological response evaluation after neoadjuvant chemotherapy, to determine if there is residual disease.

\section{B. Core needle and vacuum-assisted core biopsy (histology)}

It is the ideal diagnostic method for non-palpable lesions; it is performed under local anesthesia. In addition to the necessary equipment and adequate training to be available, it is important mentioning that its main application is in calcifications' biopsy.

In BI-RADS category 4 and 5 lesions, either nodules or microcalcifications, there is the alternative of US or stereotactic-guided biopsy with vacuum-assisted cutting systems, with the latter being indispensable for microcalcifications. A metal clip needs to be placed at the biopsy site.

Microcalcification extraction is corroborated with X-ray of the fragments, prior to histopathological examination.

Surgical biopsy with diagnostic and therapeutic purposes is required when in the core needle biopsy and/ or vacuum-assisted core biopsy histopathological result there is no correlation between imaging and pathology or when histopathological study considers excision.
Radiological control of the intervened breast is necessary in a 6-month period.

In all cases, the correlation between imaging and pathology results should guide the treatment; breast cancer management interdisciplinary groups shall have a systematic working method that enables clinician, radiologist and pathologist correlation. ${ }^{1}$

The criteria for the selection of the type of biopsy are described in table 1.

\section{Histopathological study}

\section{Recommendations for conservative surgery specimen handling and report}

I. Trans-operative indications:

a) Surgical margins status.

b) Sentinel lymph node.

II. Specimen management:

a) The specimen must be referred with radiological study.

b) Margins referred (6) with silk suture, beads or staining (ideally stained by the surgeon).

c) The surgical specimen should be received intact (without any type of manipulation or section).

d) The specimen should be sectioned only by the pathology physician.

e) Surgical margins perpendicular sections (a surgical margin is regarded as negative for ductal carcinoma in situ when it is $>2 \mathrm{~mm}$ distant). ${ }^{1}$

f) Specimen 2 to 3-mm-thick serial sections.

g) Include the sections in a serial and ordered manner. If the specimen has a wire marker, refer the number of capsules of the marked lesion. 
h) Including the totality of tissue marked by the wire plus $1 \mathrm{~cm}$ of the periphery is recommended, which is representative of the remaining tissue.

i) Indicate the list of sections in the macroscopic description.

\section{Recommendations for infiltrating breast carcinoma histopathological report}

This Consensus recommends the AJCC protocol for breast cancer patients' specimens' examination. The diagnostic parameters we consider to be essential in the histopathology report are:

I. Type of procedure: diagnostic or therapeutic and anatomical localization.

II. Macroscopic parameters:

a) Tumor weight and size on its three dimensions.

b) Type of margins: infiltrating and non-infiltrating.

c) Tumor distance from margins and surgical bed, referred by the surgeon.

III. Microscopic parameters:

a)Histological type: histological type diagnosis should adhere to the criteria of the WHO Breast Tumors Classification, $4^{\text {th }}$ edition. ${ }^{3}$

In case different patterns are observed, specify the percentage of each one of them.

b) Histological grade: infiltrating ductal carcinoma and all its variants, except for medullary carcinoma, should be graded with the Scarff-Bloom-Richardson (SBR) grading system, as described below:

- Tubule formation:

- Score 1: $75 \%$ or more of tumor composed of tubules.

- Score 2: 10 to $75 \%$ of tumor composed of tubules.

- Score 3: less than $10 \%$ of tumor composed of tubules.

- Nuclear grade:

- Score 1: small, uniform nucleus, dense chromatin.

- Score 2: nucleus with size and shape moderate variation; scarcely apparent nucleolus can be observed.

- Score 3: nucleus with marked increase in size, irregular shape and contour, 2 or more prominent nucleoli, thick chromatin.

- Mitotic count:

- Score 1: less than or equal to 3 mitoses per $\mathrm{mm}^{2}$.

- Score 2: 4 to 7 mitoses per $\mathrm{mm}^{2}$.

- Score 3: equal to or greater than 8 mitoses per $\mathrm{mm}^{2}$. ${ }^{*}$ See target conversion table in reference 3.
Adding the number of mitoses per 10 high power fields (400x) is also recommended.

- The three above mentioned parameters shall be reported separately, as well as the final score to determine the histological grade, which will be as follows:

- Grade I: 3 to 5 points.

- Grade II: 6 to 7 points.

- Grade III: 8 to 9 points.

Lobular carcinoma should be evaluated with the modified SBR scale. ${ }^{4}$

III. In the presence of ductal carcinoma in situ or intralobular neoplasm, mention the type and percentage.

IV. Lymphovascular permeation evaluated in peritumoral tissue.

V. Skin, nipple and areola (ulcerated papillary, reticular dermis) and muscle infiltration.

VI. Tumor infiltrating lymphocytes (TIL) assessment will be carried out following the TILs Working Group 2014 recommendations. ${ }^{5}$ The percentages are reported in three groups: $1-19 \%$ low, $20-49 \%$ intermediate and $\geq 50 \%$ high.

VII. Report the presence of microcalcifications in core needle biopsies, stereotactic biopsies and conservative surgery specimens.

VIII. Other associated entities (hyperplasia, columnar cells, microglandular adenosis, etc.).

IX.Axillary dissection:

a) Specify total dissected lymph nodes.

b) Number of lymph nodes with metastasis.

c) Size of dissected lymph nodes.

d)Capsular rupture and periganglionar soft tissue infiltration by neoplastic cells.

\section{Recommendations for post-treatment specimen report}

Reporting post-treatment specimens is recommended using the residual cancer burden (RCB) index, ${ }^{6}$ which has demonstrated to be a good disease-free survival predictor. To determine it, the following information is required:

I. Residual tumor size (two dimensions in $\mathrm{mm}$ ).

II. Invasive carcinoma cellular density.

III. Number of positive lymph nodes.

IV.Diameter $(\mathrm{mm})$ of the largest metastasis to lymph nodes.

The information is integrated to a mathematical formula online (www.mdanderson.org/breastcancer_RCB) 
Table 1. Classes of treatment response according to the M.D. Anderson Center Residual Cancer Burden (RCB) index

\begin{tabular}{|l|l|l|}
\hline CLASS & RESPONSE & $\infty$ \\
\hline RCB 0 & Pathological complete response (no invasive carcinoma or lymph node metastasis) & $\frac{\infty}{5}$ \\
\hline RCB 1 & Partial response, minimal residual disease & $\frac{1}{0}$ \\
\hline RCB 2 & Partial response, moderate residual disease & $\frac{1}{\sigma}$ \\
\hline RCB 3 & Chemoresistant, minimal response or no response & $\frac{5}{0}$ \\
\hline
\end{tabular}

Table 2. Recommendations for papillary neoplasms histopathological report

\begin{tabular}{|c|c|c|c|c|}
\hline & CK 5/6 & ER & P63, SMA or calponin & \\
\hline $\begin{array}{l}\text { ENCAPSULATED OR INTRACYSTIC } \\
\text { PAPILLARY CARCINOMA }\end{array}$ & Negative & Intense positive & Absent in tumor periphery and & center \\
\hline $\begin{array}{l}\text { SOLID PAPILLARY CARCINOMA } \\
\text { - In situ } \\
\text { - Invasive }\end{array}$ & Negative & Intense positive & Absent in tumor periphery and & $\begin{array}{c}\text { center } \\
\circ \\
\frac{c}{0}\end{array}$ \\
\hline $\begin{array}{l}\text { INTRADUCTAL PAPILLOMA } \\
\text { - Atypical (area of atypia } \leq 3 \mathrm{~mm} \text {; } \\
\text { focus } \geq 3 \mathrm{~mm} \text { is regarded as } \\
\text { DCIS-associated papilloma) } \\
\text { - With DCIS } \\
\text { - With LCIS }\end{array}$ & $\begin{array}{l}\text { Positive (mosaic pattern) } \\
\text { Negative in areas of } \\
\text { carcinoma }\end{array}$ & Weak positive and focal & $\begin{array}{l}\text { Present in lesion periphery an } \\
\text { Negative in areas of carcinom }\end{array}$ & d. center \\
\hline
\end{tabular}

to calculate a numerical value that determines four categories (Table 1).

To perform a complete evaluation and guide post-treatment specimen sampling, the pathologist must have the following information: tumor size prior to treatment, multifocality or multicentrality, location, clinical signs of inflammatory carcinoma, relationship with skin and chest wall, previous biopsy results, including immunohistochemistry $(\mathrm{IHC})$ markers, as well as post-treatment clinical and radiological assessment.

Placement of a metal clip prior to treatment is recommended in order to ensure tumor bed identification.

\section{High grade precursor lesions and breast carcinoma in situ histopathological report}

I. Recommendations for intracystic papillary carcinoma and related papillary neoplasms histopathological report. $^{7}$

a) The diagnostic criteria are established in table 2.

b) If in intracystic papillary carcinomas there are invasion foci, only the infiltrating component size should be reported for staging purposes.

c) Issuing papillary neoplasms definitive diagnoses trans-operatively, in core needle biopsy and in aspiration biopsy is contraindicated.

II. Lobular carcinoma in situ can be associated with tubular carcinoma and columnar cell lesions (Rosen triad). ${ }^{8}$ a) Columnar cell diagnosis as precursor lesion can be established following the flow chart shown in figure $1 .^{9}$

III. Triple-negative carcinoma and association with microglandular adenosis.

Microglandular adenosis (MGA) is considered to be a benign ductal proliferation, but in $27 \%$ of cases there is significant risk for the development of invasive carcinoma in situ of the basal type (triple-negative). Therefore, MGA detection and certainty diagnosis are important and include the following IHC panel: S-100-positive, ER-negative and p63-positive. ${ }^{10,11}$

IV. Recommendations for ductal carcinoma in situ report. ${ }^{12-14}$

a)Anatomo-radiological correlation.

- Mammographic characteristics of the specimen (microcalcifications, density alteration).

b) Tumor size:

- Multiply the number of slides with tumor by $4 \mathrm{~mm}$.

- Measure the longest diameter.

- Tumor size will be taken as the largest of both these measurements.

c) Histological grade

- Nuclear grade

- Grade 1:

- Monotonous nuclei.

- From 1.5 to 2-fold the size of an erythrocyte or an epithelial cell nucleus.

- Diffuse chromatin.

- Occasional nucleoli and mitosis. 


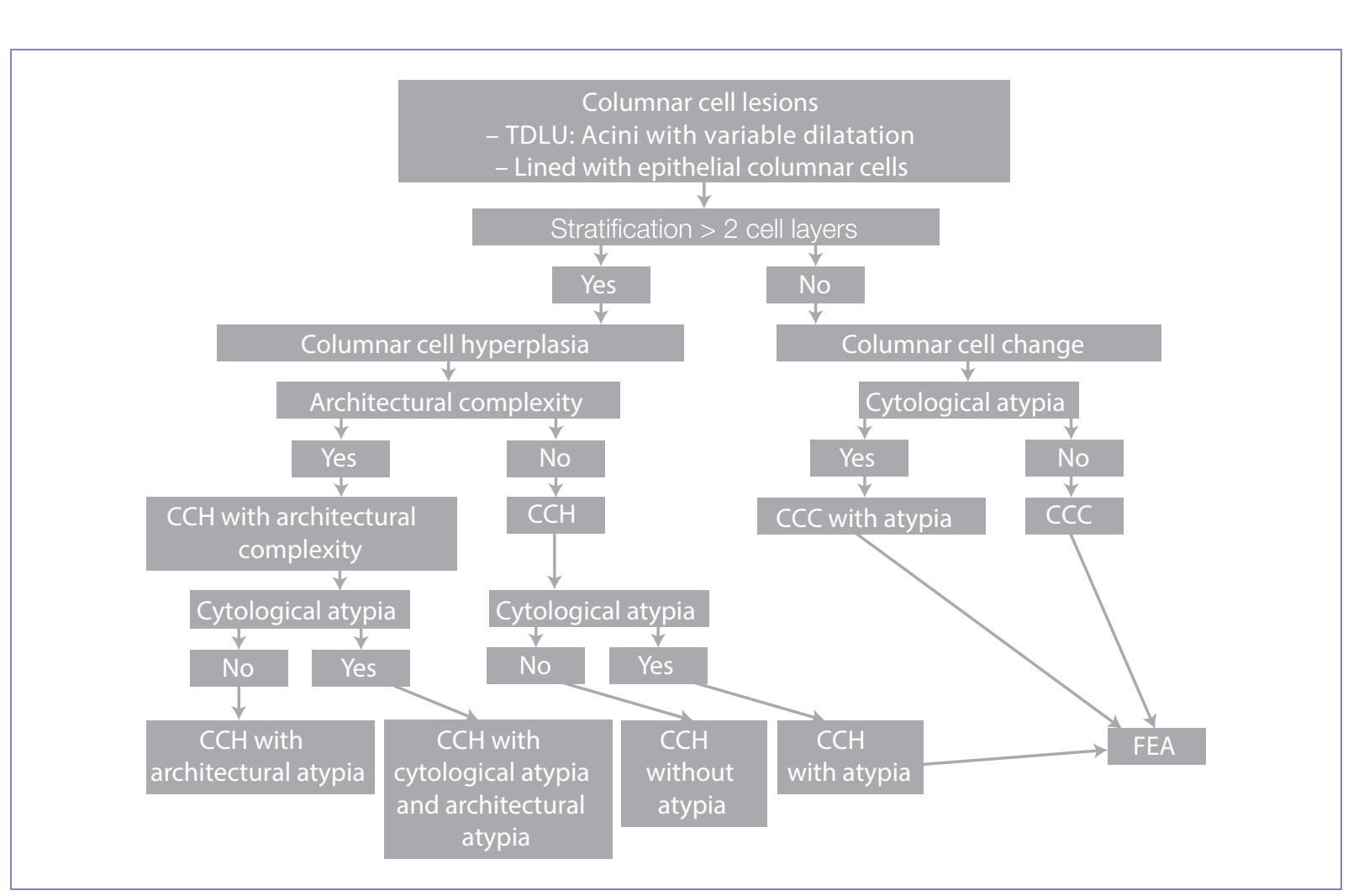

Figure 1. Algorithm for columnar cell papillary lesions diagnosis.

- Cell polarization.

- Grade 2:

- Moderate pleomorphism.

- 2 to 2.5 -fold the size of an erythrocyte or an epithelial cell nucleus.

- Fine to coarse chromatin.

- Evident nucleolus and sparse mitoses.

- Grade 3:

- Marked pleomorphism.

- More than 2.5-fold the size of an erythrocyte or an epithelial cell nucleus.

- Prominent nucleoli.

- Abundant mitoses.

- Absent or present necrosis.

- Architectural patterns:

- Comedo

- Cribriform.

- Papillary.

- Micropapillary.

- Solid.

- Infrequent variants:

- Apocrine cells.

- Cystic hypersecretory.
- Mucocele type.

- Signet ring cells.

- Small cells.

- Squamous cell type.

- Papillary lesions:

- Complex or atypical papilloma.

- Papilloma complicated with carcinoma in situ.

d) Surgical margins:

- Specify the distance between the ductal carcinoma in situ (DCIS) closest focus and the inked margin. If positive, report if focal or diffuse (surgical margin is considered to be positive for ductal carcinoma in situ when $>2 \mathrm{~mm}$ away). ${ }^{1}$

e) Microcalcifications:

- Carcinoma in situ-associated.

- Adjacent to area of carcinoma in situ.

f) The report should include the sum of variables used in the Van Nuys prognostic index..$^{15-17}$

g) Other parameters:

Hormone receptor status determination with report that must include the percentage of positive neoplastic cells. In the consensus, HER-2 neu determination was not 


\begin{tabular}{|l|c|}
\hline 100\% Formalin & 1 liter \\
\hline Distilled water & 9 liters \\
\hline Sodium phosphate, monobasic & 40 grams \\
Sodium phosphate, dibasic & 65 grams \\
\hline
\end{tabular}

Figure 2. Buffered formalin formula ( $\mathrm{pH}$ (6.8).

considered relevant for ductal carcinoma in situ; however, it can be carried out for investigational purposes.

h) Microinvasive carcinoma. ${ }^{18}$

The term microinvasive carcinoma refers to the presence of DCIS in which there is a rupture of the basal membrane and a microscopic infiltration focus of up to $1 \mathrm{~mm}$, single invasive carcinoma focus $<2 \mathrm{~mm}$ or else three invasive foci of $<1 \mathrm{~mm}$ each.

\section{Recommendations for sentinel lymph node histopathological report}

Sentinel lymph node (SLN) evaluation includes:

I. Trans-operative procedure: $:^{19,20}$

a) Lymph node serial longitudinal sections every $2 \mathrm{~mm}$.

b) Cytology evaluation by apposition or imprint of each face.

II. Ten definitive sections in paraffin, serial, with 200-micra interval ${ }^{21}$ and IHC (cytokeratins AE1/AE3) in section \#5, only in selected cases or with lobular carcinoma.

III. Histopathological report;

a)Lymph node negative for metastasis by hematoxylin-eosin (H-E) and IHC.

b) Positive lymph node with macrometastasis (metastases larger than $2 \mathrm{~mm}$ ).

c) Positive lymph node with micrometastases of $0.2 \mathrm{~mm}$ to $2 \mathrm{~mm}$ on largest dimension. Document if detected by $\mathrm{H}-\mathrm{E}$ or $\mathrm{IHC}$.

d) Positive lymph node with isolated tumor cells (single cells or small nests not larger than $0.2 \mathrm{~mm}$ ). Document if detected by $\mathrm{H}-\mathrm{E}$ or $\mathrm{IHC} .^{22}$

e) Report capsular rupture and size of extent to adipose tissue. ${ }^{22}$

\section{Recommendations for breast tumor fine needle aspiration biopsy (FNAB) report}

The Consensus does not recommend making therapeutic decisions based on primary tumor cytopathological diagnosis.

\section{Recommendations for the report of axillary lymph node FNAB with possible metastasis}

I. Positive for metastasis.

II. Negative for metastasis.

III. Insufficient for diagnosis.

\section{Recommendations for the report of prognostic-predictive factors by immunochemistry}

Hormone (estrogen and progesterone) receptor status and protein HER-2, Ki67 overexpression are indispensable prognostic and predictive factors in breast factors, and all patients with this diagnosis should therefore have these markers determined. ${ }^{23,24}$

I. Tissue management:

a) $10 \%$ buffered formalin should be used as fixative, and it can be purchased of prepared in the pathology laboratory with the formula described in figure 2 .

b) The tissue should be placed in the fixative as soon as possible, no more than 15 minutes after surgery.

c) The tissue should be sliced in 2 to $5 \mathrm{~mm}$-thick sections and in the case of tru-cut biopsy, including 2 cylinders per capsule is recommended owing to breast cancer recognized heterogeneity.

d) The ratio between sample volume and fixative should be 20 to 1 .

e) Fixation is recommended for at least 6 hours and for no more than 48 hours; in order to avoid prolonged fixation, it is desirable for the specimen to be changed to buffering solution before reaching 48 hours.

f) Determination of hormone receptors, HER-2 neu and $\mathrm{Ki}-67$ is indicated in the primary tumor, residual tumor and metastases.

II. Interpretation criteria

a) The following guidelines decrease the possibility of incorrect interpretations: ${ }^{25}$ 
Validated antibody clones should be employed:

- Clones for estrogen receptors: 1D5, 6F11, SP1, ID5+ER.2.123.

- Clones for progesterone receptors: 1A6, 1294, 312.

- Clones for HER-2: 4D5, CB11, A085.25.

- Positive and negative controls should always be examined. There should be no unspecific staining in the control or in the problem case (e.g. HER-2 neu-positive healthy tissue).

- Interpret each staining only in samples with more than $0 \%$ of well-preserved tissue. Minimal recommended tumor area for marker reliable assessment is equivalent to 2 tru-cut biopsy cylinders with at least $60 \%$ of viable neoplastic tissue.

b) Estrogen and progesterone receptors (ER and PR, respectively) are positive when expressed as nuclear staining.

The $\mathrm{H}$-score and Allred systems are recommen$\operatorname{ded}^{26,27}$, specifying the percentage of positive cells.

- H-score system:

$-\%$ of positive cells $\times 3$ (intense nuclear staining), plus

- \% of positive cells $\times 2$ (moderate nuclear staining), plus

- $\%$ of positive cells $x 1$ (weak nuclear staining).

The result is the $\mathrm{H}$-score index, which ranges from 0 to 300 .

- Allred system:

Positive area with higher staining intensity calculated as follows:

- Positive area

- 0: No positive cells.

$-1:<1 \%$ positive cells.

-2: 1 to $10 \%$ positive cells.

- 3: 11 to $33 \%$ positive cells.

$-4 ; 34$ to $66 \%$ positive cells.

- 5: $67 \%$ or more positive cells.

- Staining intensity: 1 = weak, 2 = moderate and $3=$ intense.

The result is the Allred index, which ranges from 0 to 8 .

- Currently, it is valid to report only the percentage of positive cells both for estrogen and progesterone receptors. Both ER and PR are considered positive with $1 \%$ of positive neoplastic cells. ${ }^{28}$

c) HER-2 overexpression: ${ }^{29,30}$

- Positive (3+): intense and uniform membrane staining in $>10 \%$ of neoplastic cells.

- Indeterminate (2+): complete and weak membrane staining in $>10 \%$ of neoplastic cells.
- Negative (0-1+): no staining is identified or it is weak and incomplete in at least $10 \%$ of neoplastic cells. In HER-2, the classification applies only for invasive carcinoma, not for carcinoma in situ. Positive cases for HER-2 in normal ducts and lobules are not evaluable and should be repeated.

d) Recommendations for Ki67 reporting:31-33

- Preanalytical:

- The Ki67 index can be determined in tru-cut biopsies and/or complete tumors in large excisions.

- The Ki67 index in tissue microarrays should only be used in clinical or epidemiological trials.

- Analytical:

- Known positive and negative controls should be included in the electrocharged slides.

- Nuclear staining is only considered positive.

- The MIB-1 antibody is the one currently accepted.

- Interpretation:

- In the panoramic view of the tumor, at least 3 high power fields (400x) representing the entire tumor staining spectrum should be selected. The evaluation is made in at least $\mathbf{5 0 0}$ neoplastic cells, with 1000 cells being most recommendable.

- In prognosis-evaluation studies, assessing tumor invasive border is recommended.

- In pharmacokinetic examinations comparing tru-cut biopsies and large excisions, assessing the entire tumor is recommended.

- A "hot-spot" is defined as an area where staining is particularly higher with regard to other adjacent areas. If there are several "hot spots", the one with the highest rank should be selected.

- Using two methods is advisable:

- Average: it consists in manually counting the number of positive cells in the three previously-selected fields and calculating the average.

- Hot spot: it consists in manually counting the number of positive cells at the highest-rank "hot spot" and calculating the average.

- Report

- The Ki67 reported index is the percentage of positive neoplastic cells among total counted cells.

- We recommend reporting the obtained index using both above-described methods: "hot spot" and "average". 
- The cutoff point recommended by this Consensus is $20 \%$.

\section{Report form}

a) The IHC report should be linked to the pathology main report in order to ensure that the results are incorporated to the final diagnosis.

b) In order to ensure for results to be reproducible, the report must include the antibody clone and brand, the status (positive or negative), as well as the used criteria and system.

IV. Routine quality control

Routine quality control is essential for $\mathrm{IHC}$ reaction success.

a) Positive and negative controls should be included in the same slide where the problem tissue is analyzed. If these controls are in a separate slide, it has to be ensured that they undergo simultaneous and identical procedures than the problem specimen.

b) The controls must be identically fixed and processed than the examined tissue and undergo the same antigen retrieval and immunostaining protocol.

c) Controls with three staining levels (negative, weak/ moderate, intense) should be used in order to obtain an adequate staining.

d)Histological sections for immunohistochemistry should be stored at room temperature for no longer than 14 days; after this period, the results are questionable. ${ }^{32}$

V. External quality control ${ }^{34}$

a) The pathology laboratories that perform IHC tests should participate in an external quality control program.

b) For an adequate IHC quality control, the laboratory is required to process the samples of at least 200 cases per year.

\section{Recommendations for molecular biology}

\section{HER-2 AMPLIFICATION}

Currently, there are different techniques to identify HER-2 gene amplification; fluorescent in situ hybridization (FISH) is considered the gold standard. Other variants of this technique are chromogenic in situ hybridization $(\mathrm{CISH})$ and silver in situ hybridization (SISH), which are techniques that can be simple (based only on HER-2 detection) or dual (based on the HER-2/ chromosome 17 centromere ratio). ${ }^{35}$
a)HER-2 amplification should be looked for in indeterminate cases (2+ positive) by IHC.

b) The $\mathrm{CISH}$ and $\mathrm{SISH}$ techniques can be used provided a validation process of them has been carried out in parallel with the FISH technique and concordance of at least $95 \%$ has been demonstrated between FISH and the other methodology.

I. HER-2 hybridization reactions interpretation criteria:

a) The following guidelines reduce the probability of interpretation errors:

- The zone with invasive carcinoma should be selected in the $\mathrm{H}$-E-stained tumor section; the test will not be performed in areas with carcinoma in situ.

- The control is initially assessed; if inadequate, the test should be repeated.

- Global assessment of the case should be made and have at least 20 neoplastic cells for SISH or $\mathrm{CISH}$ and 40 for $\mathrm{FISH}$ in at least two different invasive carcinoma fields. In case there are areas with and without amplification, they should be separately counted. It should be reported as amplified with a note specifying that there are zones without amplification. ${ }^{29,30}$

II. Cutoff points for dual FISH and SISH:

a) Positive: HER-2/CEP 17 ratio $>2.0$

b) HER-2/CEP $17<2$ but with HER-2 absolute count per nucleus $>6$.

c) Indeterminate: HER-2/CEP 17 ratio $<2$ but with HER-2 absolute count per nucleus $\geq 4$ and $<6$.

d) Negative: HER-2/CEP 17 ratio $<2$ and absolute count $<4$.

III. Cutoff points for simple CISH

a)Positive: 6 > copies/nucleus.

b) Indeterminate: 4 to 6 copies/nucleus (in two counts).

c) Negative: < 4 copies/nucleus.

Note: Using preferably dual systems is recommended.

\section{MOLECULAR CLASSIFICATION OF BREAST CARCINOMA AND ITS IMMUNOHISTOCHEMICAL APPROACH}

Translational medicine works on breast cancer four molecular phenotypes (luminal, with HER-2 overexpression, basal phenotype and normal breast-like), which initially were defined by genomics, ${ }^{36}$ have enabled approaching this classification by means of more accessible methodologies such as IHC by using routine markers such as ER, PR and HER-2. ${ }^{37-40}$ In the Mexican population, mean frequency of subgroups defined by this markers is as follows: hormone receptor-positive $60 \%$, HER-2-positive $20.4 \%$ and triple-negative $23.1 \%{ }^{41,42}$ Breast cancer 
Table 3. Breast cancer molecular subtypes and their IHC approach according to this consensus.

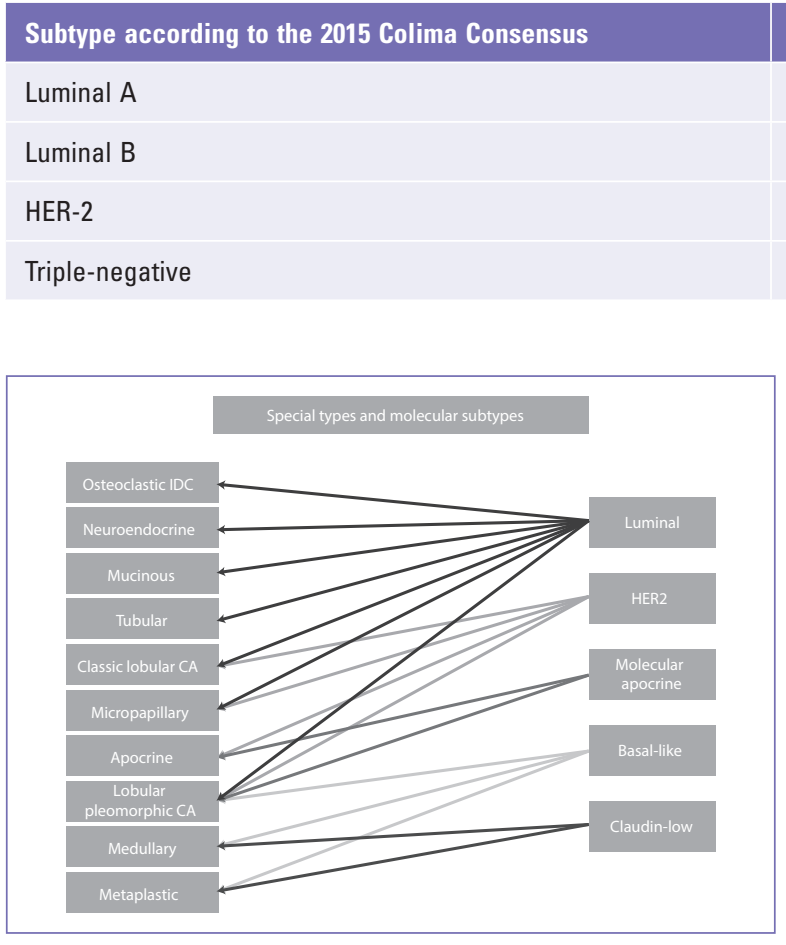

Figure 3. Special types and molecular subtypes.

molecular subtypes and their approach by $\mathrm{IHC}$ according to this Consensus are shown in table $3{ }^{43-45}$

\section{TRIPLE-NEGATIVE BREAST CANCER}

Triple-negative (TNBC) and basal-like breast cancer should not be considered synonyms, since only $49 \%$ to $71 \%$ of TNBC is basal-like and $77 \%$ of basal-like is triple-negative. ${ }^{46,47}$ TNBC has been sub-classified by gene expression in different ways: a) enriched HER-2 neu, basal-like and claudin low, ${ }^{42}$ basal 1 and basal 2 (BL1 and BL2), mesenchymal (M) and mesenchymal stem-like (MSL), immunomodulatory (IM) and luminal androgen receptor subtype (LAR). ${ }^{48,49}$

The following IHC panel is recommended for TNBC in order to favor biomarkers and patient subgroups identification:

a)Basal cytokeratins (ck5/6, ck14 and ck17).

b) EGFR.

c) $\mathrm{P} 53$

d)Androgen receptors.

Classification of triple-negative tumors

a) Low histological grade:

- Adenoid cystic.

- Secretory.

\section{Immunohistochemical approach}

$\mathrm{ER}+, \mathrm{PR}>20 \%$, Ki67 $<20 \%$ HG 1 or 2 and HER2 -

$\mathrm{ER}+, \mathrm{PR}<20 \%, \mathrm{Ki} 67<20 \% \mathrm{HG} 1$ or 2 and HER2 + or -

HER2 +, ER and PR -

$\mathrm{ER}-, \mathrm{PR}-$ and HER2 -

- Metaplastic.

- Metaplastic, fibromatosis-like.

- Adenosquamous.

b) Intermediate histological grade:

- Medullary carcinoma.

c) High histological grade:

- Metaplastic.

- Neuroendocrine.

- DIC, NOS.

\section{SPECIAL TYPES}

Group of carcinomas with different morphological characteristics, biological behavior and clinical evolution to ductal infiltrating carcinoma NOS, which in addition account for $25 \%$ of all breast carcinomas. ${ }^{50,51}$ Special types in correlation with molecular subtypes are shown in figure 3 and table 4 . In secretory carcinoma and adenoid cystic carcinoma, characteristic genetic alterations have been identified and, currently, demonstrating them is a requirement to obtain diagnostic certainty in these entities.

a) Secretory carcinoma must have $t(12 ; 15)(p 13 ; q 25)$ with ETV6-NTRK3.44 fusion gene.

b) Adenoid cystic adenoma must have $t(6 ; 9)$ (q2223;p23-24) with MYB-NFIB fusion gene.

In cases of difficult-to-diagnose lobular carcinoma, use e-cadherin, $B$ catenin and $p 120 .^{52}$

\section{Molecular biology in ductal CARCINOMA IN SITU CLASSIFICATION}

Molecular study of ductal carcinoma in situ and other breast cancer precursor lesions has revealed the existence of two molecular pathways in breast oncogenesis (Tables 5 and 6). The first one gives origin to low-grade invasive carcinomas and involves change and hyperplasia of columnar cells with cytological atypia, atypical ductal hyperplasia and low-grade ductal carcinoma in situ. 
Table 4. Molecular subtype characteristics and breast cancer special histological types' assignment.

\begin{tabular}{|c|c|c|c|c|c|}
\hline Molecular subtype & ER, PR, HER2 & Additional marker & Proliferation microarrays & \multicolumn{2}{|c|}{ Special histological type } \\
\hline BASAL-LIKE & $\begin{array}{l}\text { ER - } \\
\text { PR - } \\
\text { HER-2 - }\end{array}$ & $\begin{array}{l}\text { CK5/6 + } \\
\text { EGFR + }\end{array}$ & High & $\begin{array}{l}\text { Adenoid cystic } \\
\text { Acinar cells } \\
\text { Medullary } \\
\text { Metaplastic } \\
\text { Lobular pleomorphic } \\
\text { Secretory }\end{array}$ & 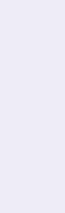 \\
\hline HER/ER- & $\begin{array}{l}\text { ER - } \\
\text { PR - } \\
\text { HER-2 + }\end{array}$ & $\begin{array}{l}\text { CK5/6 +/- } \\
\text { EGFR +/- }\end{array}$ & High & $\begin{array}{l}\text { Apocrine } \\
\text { Lobular } \\
\text { Micropapillary } \\
\text { Lobular pleomorphic }\end{array}$ & () \\
\hline NORMAL BREAST-LIKE & $\begin{array}{l}\text { ER -l+ } \\
\text { PR unknown } \\
\text { HER - }\end{array}$ & $\begin{array}{l}\text { CK5/6 } \\
\text { EGFR + }\end{array}$ & Low & $\begin{array}{l}\text { Medullary } \\
\text { Metaplastic }\end{array}$ & $\begin{array}{l}\frac{c}{\frac{c}{2}} \\
\frac{2}{3} \\
\frac{2}{1}\end{array}$ \\
\hline LUMINAL & $\begin{array}{l}\mathrm{ER}+(-) \\
\mathrm{PR}+/- \\
\mathrm{HER}-(+)\end{array}$ & & Low/high & $\begin{array}{l}\text { Apocrine } \\
\text { Osteoclastic ductal car } \\
\text { Lobular } \\
\text { Micropapillary } \\
\text { Mucinous } \\
\text { Neuroendocrine } \\
\text { Lobular pleomorphic } \\
\text { Tubular }\end{array}$ & 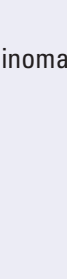 \\
\hline MOLECULAR APOCRINE & $\begin{array}{l}\text { ER - } \\
\text { PR - } \\
\text { HER2 +/- }\end{array}$ & $\begin{array}{l}\mathrm{AR}+ \\
\mathrm{CK} 5 / 6+/- \\
\mathrm{EGFR}+/-\end{array}$ & High & $\begin{array}{l}\text { Apocrine } \\
\text { Lobular pleomorphic }\end{array}$ & $\begin{array}{l}\frac{1}{2} \\
\frac{.}{3} \\
\frac{1}{6}\end{array}$ \\
\hline CLAUDIN-LOW & $\begin{array}{l}\text { ER - } \\
\text { PR - } \\
\text { HER-2 - }\end{array}$ & $\begin{array}{l}\text { CDLN-low/- } \\
\text { CDH1-low/- } \\
\text { CK5/6 +/- } \\
\text { EGFR +/- }\end{array}$ & High & $\begin{array}{l}\text { Metaplastic } \\
\text { Medullary (?) }\end{array}$ & 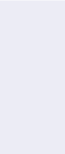 \\
\hline INTERFERON-RELATED & $\begin{array}{l}\text { ER -l+ } \\
\text { PR unknown } \\
\text { HER-2 - }\end{array}$ & STAT1 & High & Medullary (?) & $\begin{array}{l}\frac{5}{3} \\
0 \\
\frac{1}{3}\end{array}$ \\
\hline
\end{tabular}

AR: Androgen receptor; CDH1: E-cadherin; CDLN: Claudin; CK: Cytokeratin; EGFR: Epidermal growth factor receptor; ER: Estrogen receptor; PR: Progesterone receptor; STAT1: Signal transductor and transcription activator 1 ; -: Negative; +: Positive; +l-: Occasional positive; -/+: Rarely positive.

The second pathway gives origin to high-grade invasive carcinomas involving high-grade carcinoma in situ without other well-identified precursors. ${ }^{53,54}$

\section{Invasive breast carcinoma molecular signatures}

Molecular signatures are a useful tool for personalized systemic treatment selection in a selected group of patients with early disease. ${ }^{55-58}$ This enables having more certainty on the usefulness of indicated treatment, thus avoiding the toxicity of a therapy with limited benefit.

Their use is exclusively recommended in patients with early disease and hormone-sensitive, HER-2-negative tumors. ${ }^{55-58}$

Currently, in Mexico there are three molecular signatures available that can be employed in this group of patients: Oncotype DX, Mammaprint and Endopredict.

\section{Oncotype DX}

Oncotype DX is a test that: i) quantifies the probability of disease recurrence in women with early-stage breast cancer with negative lymph nodes, positive ER, normal HER-2 (prognostic significance) and ii) evaluates the possible benefit of a particular treatment, chemotherapy or hormone therapy (predictive significance). ${ }^{59,60}$ Oncotype DX analyses a panel of 21 tumor genes to determine a recurrence score (RS $\leq 18$ : low, 18 to 30 : intermediate and $\geq 31$ high).

The TAILORx trial results will provide definitive information in patients with negative lymph nodes, positive hormone receptors and negative HER-2 with intermediate recurrence risk (RS 10-25), on the benefit of adjuvant chemotherapy + hormone therapy vs. hormone therapy. Initial results of the trial demonstrated that those patients with recurrence score $<11$ have an excellent 
Table 5. Ductal carcinoma in situ oncogenic pathways.

\begin{tabular}{|l|l|l|l|l|}
\hline Grade & Cytogenetic alterations & Histopathological data & Evolution \\
\hline Low grade & $\begin{array}{l}\text { Genomic alterations' simple pattern } \\
16 q \text { loss } \\
1 q \text { gain }\end{array}$ & $\begin{array}{l}\text { Small grade I nuclei } \\
\text { Absence of necrosis } \\
\text { Positive hormone receptors }\end{array}$ & $\begin{array}{l}\text { Long period of } \\
\text { time } \\
10 \text { to } 20 \text { years }\end{array}$ & $\begin{array}{l}\text { Well-differentiated } \\
\text { invasive carcinoma }\end{array}$ \\
\hline High grade & $\begin{array}{l}\text { Genomic alterations' complex pattern } \\
16 q, 11 q, 14 q, 8 p, 13 q \text { and } 18 q \text { loss } \\
1 q, 17 q, 8 q, 20 q \text { and } 5 p \text { gain } \\
17 q 12,17 q 22-24,6 q 22,8 q 22,11 q 13 \text { and } \\
20 q 13 \text { amplification }\end{array}$ & $\begin{array}{l}\text { High nuclear grade } \\
\text { Presence of comedo-necrosis } \\
\text { Negative hormone receptors, } \\
\text { positive HER-2 neu }\end{array}$ & $\begin{array}{l}\text { Short time period } \\
2 \text { to } 5 \text { years }\end{array}$ & $\begin{array}{l}\text { Poorly differentiated } \\
\text { invasive carcinoma }\end{array}$ \\
\hline
\end{tabular}

Table 6. Ductal carcinoma in situ prognosis according to genetic alterations.

\begin{tabular}{|l|l|l|}
\hline Genetics & Gene expression analysis & Prognosis \\
\hline $6 \mathrm{p} 21-25$ gain & $\begin{array}{l}\text { Poor prognosis } \\
\text { Risk for metastasis }\end{array}$ & Poor \\
\hline $17 q 21$ gain & & Poor \\
\hline $16 q$ loss & Good prognosis & Good \\
\hline $17 q 21$ gain & & Intermediate \\
\hline
\end{tabular}

prognosis, with a 5-year distant recurrence-free survival rate of $99.3 \%{ }^{61}$

\section{MAMMAPRINT}

Mammaprint is a genomic test to assess the metastatic risk of a breast tumor. Mammaprint is based on the genomic signature of 70 genes by using fresh tissue for microarray and paraffin-embedded tissue analysis. $62-64$

MINDACT trial initial results demonstrated that, in patients considered of high clinical risk, but with a Mammaprint low result, the 5-year metastasis-free survival rate was $94.7 \%$ in those who received no chemotherapy. ${ }^{65}$

\section{ENDOPREDICT}

It is a genomic expression signature, validated to measure the probability of distant recurrence in patients with early breast cancer with positive hormone receptors, negative HER-2 treated with adjuvant endocrine therapy. ${ }^{66-68}$ The test quantitatively analyses messenger RNA levels of 8 cancer-related genes of interest and three reference genes by means of qRT-PCR in formalin-fixed, paraffin-embedded tumor tissue. A recurrence risk score was developed, and its subsequent combination with nodal status and tumor size allows for a comprehensive risk score to be obtained (EPclin). The cutoff point was established at 3.3 for EPclin to categorize patients at low or high risk.

For any of the signatures, omitting chemotherapy treatment is recommended in patients classified as low-risk. ${ }^{61,65,67}$

The use of genomic signatures in patients with positive lymph nodes is currently not currently recommended. ${ }^{57}$

\section{Participation of the pathologist in genomic SIGNATURE STUDIES}

Currently, genomic signature testing is made in a centralized manner at specialized laboratories. Participation of the pathologist is highly important for adequate selection of the material required for the tests, and observing the following points is therefore recommended;

a) Use only specimens that in their processing have been fixed in $10 \%$ buffered formalin.

b) Annex complete and adequate diagnosis including immunohistochemistry markers according to the signature to be used.

c) There is no sufficient information with regard to tumor minimum extent criteria. In general, specimens with tumor covering at least one 10x field will be adequate.

d) Specimens not adequate for processing:

- MammaPrint; samples with less than $30 \%$ of tumor

- Oncotype: specimens only with tumor foci smaller than $1 \mathrm{~mm}{ }^{69}$

e) Avoid selecting blocks containing large areas of necrosis or hemorrhage.

f) Select blocks less than 5 years' old. 


\section{TNM classification ${ }^{1}$}

\section{Primary tumor}

\section{Primary tumor}

TX Primary tumor cannot be assessed.

T0 No evidence of primary tumor.

Tis Carcinoma in situ.

Tis Ductal carcinoma in situ.

(DCIS)

Tis Lobular carcinoma in situ.

Tis Paget disease of the nipple NOT related to invasive (Paget) carcinoma or carcinoma in situ (DCIS or LCIS) in the underlying breast parenchyma. Carcinomas in the breast parenchyma associated with Paget disease are categorized based on the size and characteristics of the parenchymal disease, although the presence of Paget disease should still be noted.

T1 Tumor $\leq 20 \mathrm{~mm}$ in greatest dimension.

T1mi Tumor $\leq 1 \mathrm{~mm}$ in greatest dimension.

T1a Tumor $>1 \mathrm{~mm}$ but $\leq 5 \mathrm{~mm}$ in greatest dimension.

T1b Tumor $>5 \mathrm{~mm}$ but $\leq 10 \mathrm{~mm}$ in greatest dimension.

T1c Tumor $>10 \mathrm{~mm}$ but $\leq 20 \mathrm{~mm}$ in greatest dimension.

T2 Tumor $>20 \mathrm{~mm}$ but $\leq 50 \mathrm{~mm}$ in greatest dimension.

T3 Tumor $>50 \mathrm{~mm}$ in greatest dimension.

T4 Tumor of any size with direct extension to the chest wall or to the skin (ulceration or cutaneous nodules).

T4a Extension to the chest wall not only including adherence or invasion to pectoral muscles.

T4b Skin ulceration and/or ipsilateral satellite nodules or edema (including peau d'orange) that does not meet the criteria for inflammatory carcinoma.

T4c Both T4a and T4b.

T4d Inflammatory carcinoma.

\section{Lymph nodes}

\section{Lymph nodes}

N Regional lymph nodes (clinical)

NX Regional lymph nodes cannot be assessed (e.g., previously removed).

No No palpable regional lymph nodes.

N1 Palpable metastasis to one or several movable ipsilateral axillary lymph node(s).
Metastases in ipsilateral axillary lymph node(s) fixed to one another or to other structures, or clinically detected in ipsilateral internal mammary lymph nodes in the absence of palpable axillary lymph node metastases.

N2a Metastases in axillary lymph node(s) fixed to one another or to other structures.

N2b Clinically apparent metastases in internal mammary lymph nodes without clinical evidence of axillary lymph node metastases.

N3 Clinically apparent metastasis in ipsilateral infraclavicular axillary lymph node(s) or in ipsilateral internal mammary lymph node(s) and in the presence of palpable axillary lymph node(s) or metastases in the ipsilateral supraclavicular region with or without lymph node involvement.

N3a Metastases in ipsilateral infraclavicular and axillary lymph node(s).

N3b Metastases in internal mammary and ipsilateral axillary lymph node(s).

N3c Metastases in ipsilateral supraclavicular lymph node(s)

\section{pN Regional lymph nodes (pathological)}

pNX Regional lymph nodes were not assessed.

pN0 No histopathology-detected metastases. No additional examination for isolated tumor cells. Isolated tumor cells are defined as single cells or small clusters no larger than $0.2 \mathrm{~mm}$, generally detected by immunohistochemistry or molecular methods, but verified by hematoxycilin and eosin. They are not necessarily evidence of malignant activity and may correspond to stromal reaction or proliferation.

pNO(i-) No histopathology-detected metastases and negative immunohistochemistry.

pNO(i+) No histopathology-detected metastases but positive immunohistochemistry. No malignant cell clusters larger than $0.2 \mathrm{~mm}$.

pNO(mol-) No histopathology- or RT-PCR-detected metastases.

pNO(mol+) No histopathology-detected metastases but positive RT-PCR results. The classification is based on axillary lymph node dissection with or without sentinel lymph nodes lymphadenectomy. The classification based only in sentinel lymph nodes dissection without complete axillary lymph nodes dissection is expressed with the suffix $\mathrm{sn}$, e.g., pNO(i+)(sn).

pN1 Metastases in 1-3 axillary lymph nodes and/or mammary lymph nodes with sentinel lymph node biopsy-detected but not clinically apparent microscopic disease.

pN1mi Micrometastasis (larger than $0.2 \mathrm{~mm}$ and no larger than $2 \mathrm{~mm}$ ).

pN1a Metastases in 1-3 axillary lymph nodes. 


\begin{tabular}{|c|c|}
\hline $\mathrm{pN} 1 \mathrm{~b}$ & $\begin{array}{l}\text { Metastasis in internal mammary lymph nodes with } \\
\text { micromtastases or macrometastases detected by } \\
\text { sentinel lymph node biopsy but without clinical } \\
\text { detection. }\end{array}$ \\
\hline $\mathrm{pN1c}$ & $\begin{array}{l}\text { Metastases in internal mammary lymph nodes } \\
\text { with microscopic disease detected by sentinel } \\
\text { lymph node biopsy but that are not clinically } \\
\text { apparent. }\end{array}$ \\
\hline $\mathrm{pN2}$ & $\begin{array}{l}\text { Metastases in } 4-9 \text { axillary lymph nodes or in } \\
\text { internal mammary lymph nodes clinically apparent } \\
\text { in the absence of axillary lymph node metastases. }\end{array}$ \\
\hline $\mathrm{pN2a}$ & $\begin{array}{l}\text { Metastases in } 4-9 \text { axillary lymph nodes with at } \\
\text { least one larger than } 0.2 \mathrm{~mm} \text { in diameter. }\end{array}$ \\
\hline $\mathrm{pN} 2 \mathrm{~b}$ & $\begin{array}{l}\text { Clinically apparent metastases in internal } \\
\text { mammary lymph nodes in the absence of axillary } \\
\text { lymph node metastases. }\end{array}$ \\
\hline pN3 & $\begin{array}{l}\text { Metastases in } 10 \text { or more axillary lymph nodes or } \\
\text { in infraclavicular lymph nodes or in internal } \\
\text { mammary lymph nodes together with one or more } \\
\text { positive axillary lymph nodes; or in more than } 3 \\
\text { positive axillary lymph nodes without clinical } \\
\text { adenopathies in internal mammary lymph nodes; } \\
\text { or with positive ipsilateral supraclavicular lymph } \\
\text { node. }\end{array}$ \\
\hline pN3a & $\begin{array}{l}\text { Metastases in } 10 \text { or more positive axillary lymph } \\
\text { nodes with at least one tumor deposit larger than } \\
2 \mathrm{~mm} \text {, or metastases to infraclavicular lymph } \\
\text { nodes. } \\
\text { Metastases to infraclavicular (level III axillary) } \\
\text { lymph nodes. }\end{array}$ \\
\hline $\mathrm{pN} 3 \mathrm{~b}$ & $\begin{array}{l}\text { Clinically evident metastases in internal } \\
\text { mammary lymph nodes in the presence of one or } \\
\text { more positive axillary lymph nodes; or in more } \\
\text { than } 3 \text { positive axillary lymph nodes with } \\
\text { microscopic metastasis of internal mammary } \\
\text { lymph nodes detected by sentinel lymph node } \\
\text { biopsy but not clinically evident (clinically } \\
\text { evident means found on clinical examination o } \\
\text { by imaging methods). }\end{array}$ \\
\hline \multirow[t]{2}{*}{$\mathrm{pN} 3 \mathrm{c}$} & $\begin{array}{l}\text { Metastases in ipsilateral supraclavicular lymph } \\
\text { nodes. }\end{array}$ \\
\hline & Distant metastases \\
\hline MX & Not evaluable. \\
\hline M0 & No distant metastases. \\
\hline M1 & Distant metastases. \\
\hline
\end{tabular}

RT-PCR: Reverse transcriptase polymerase chain reaction.

\section{Staging}

\begin{tabular}{|l|l|l|l|}
\multicolumn{5}{|l|}{ Staging } & N & M \\
\hline Stage & T & NO & MO \\
\hline 0 & Tis & No & MO \\
\hline IA & T1 (includes T1 mi) & N
\end{tabular}

\begin{tabular}{|c|c|c|}
\hline \multirow[t]{2}{*}{ IB } & TO & N1mi \\
\hline & T1 (includes T1 mi) & N1mi \\
\hline \multirow[t]{3}{*}{ IIA } & TO & N1 \\
\hline & T1 (includes T1 mi) & N1 \\
\hline & $\mathrm{T} 2$ & No \\
\hline \multirow[t]{2}{*}{ IIB } & $\mathrm{T} 2$ & N1 \\
\hline & T3 & NO \\
\hline \multirow[t]{5}{*}{ IIIA } & TO & N2 \\
\hline & T1 (includes T1 mi) & N2 \\
\hline & T2 & N2 \\
\hline & T3 & N1 \\
\hline & T3 & N2 \\
\hline \multirow[t]{3}{*}{ IIIB } & T4 & NO \\
\hline & T4 & N1 \\
\hline & T4 & N2 \\
\hline IIIC & Any $\mathrm{T}$ & N3 \\
\hline IV & Any $T$ & Any N \\
\hline
\end{tabular}

After the Consensus meeting, the changes of the $8^{\text {th }}$ edition of the American Joint Committee on Cancer (AJCC) guidelines for breast cancer staging were published, ${ }^{2}$ which have been proposed to be implemented as of January 1, 2018.

In this regard, and for the purposes of the present consensus, we will refer that, in addition to the factors contemplated in previous editions, such as $\mathrm{T}$ (tumor), $\mathrm{N}$ (lymph node status) and $\mathrm{M}$ (metastasis), immunohistochemistry-determined biological factors (estrogen receptors, progesterone receptors and HER-2 overexpression) and genomic tests results (Oncotype DX or others) will have to be included in order to more precisely characterize risk groups. In addition, the decision was made to eliminate lobular carcinoma in situ since it does not correspond to a malignant lesion and is merely a risk marker.

\section{Breast cancer treatment and interdisciplinary management}

Breast cancer treatment is complex and requires the participation of a multidisciplinary team in order to be able to offer patients with this diagnosis an optimal treatment. Both surgical and medical oncologists, as well as radio-oncologists, pathologists and the remaining specialists who were present in the meeting agreed on stressing on the importance of this collaborative work. 
In our country, there is evidence that cancer patient's treatment delays are common and are associated with presentations at more advanced clinical stages. These delays have been observed to start even from the moment malignancy is suspected ${ }^{1}$ and, for this reason, as professionals responsible of health services, and as a multidisciplinary team, it's our obligation to ensure that all patients receive optimal and early-administered management.

\section{Carcinoma in situ}

\section{Ductal carcinoma in situ (DCIS)}

This is a heterogeneous group of neoplasms characterized by the presence of malignant epithelial cells that grow within the mammary ducts, without surpassing the basal membrane, and are identified by light microscopy. It adopts different intraductal growth architectural patterns and exhibits variable cytological and necrotizing characteristics; generally, it is unifocal. It is also known as intraductal carcinoma.

These carcinomas are initially suspected by an abnormal mammographic finding (microcalcifications, a mass or dense asymmetric area) or by the existence of a palpable lump or secretion from the nipple. An infrequent form of presentation can be Paget disease.

Histological diagnosis and extent determination (size) are indispensable for the selection of adequate therapeutics and, therefore, many times, especially in small lesions, treatment will be administered in two times. Surgical specimen X-ray is a useful method to verify complete excision of the lesion. The dissected surgical specimen shall always be marked to accurately know each one of the margins (superior, inferior, internal, external, superficial and deep). Intraductal carcinoma is known to often grow within the ducts in a discontinuous form, and that the extent is often greater than that visualized in the mammography or clinically estimated.

\section{Recommendations for local and regional treatment}

Pathological margins smaller than $2 \mathrm{~mm}$ are considered suboptimal; the excision final pathological margin is considered positive or close when it is $\leq 2 \mathrm{~mm}$ and negative when $>2 \mathrm{~mm}^{1}{ }^{1}$ In case of surgical bed with fascia, it is regarded as optimal.

Recommendations for re-excision:

- Margin smaller than $2 \mathrm{~mm}$.

- Residual microcalcifications.
If an adequate margin is not achieved, mastectomy will be carried out. ${ }^{2,3}$ In the cases treated with conservative surgery, radiotherapy will be administered only to the breast, at a 50 Gy dose. ${ }^{4-6}$ The benefit of radiotherapy is not of the same magnitude in all patients and, therefore, they should be informed on the risks and outcomes. Radiotherapy to lymph node areas is not indicated. 4,5

Recommendations for total mastectomy:

- Multicentric disease.

- Unfavorable breast-tumor ratio.

- Impossibility to obtain 2-mm margins.

- Diffuse microcalcifications visible on mammography.

- Desire of the patient.

- Impossibility to administer radiotherapy.

\section{Sentinel lymph node in carcinoma in situ}

Generally, no axillary resection or lymphatic mapping procedure in search of sentinel lymph node is required; however, in those patients who will require mastectomy for their management or in those in whom invasion is suspected, the lymphatic mapping procedure, as well as sentinel lymph node localization and histological analysis should be carried out and act accordingly to the result.

Patients in whom microinvasion or invasion is identified in the definitive histological examination will be treated according to stage I guidelines.

\section{Treatment with tamoxifen and aromatase inhibitors}

Tamoxifen $(20 \mathrm{mg} / \mathrm{day})$ is recommended for 5 years as relapse risk-reducing therapy in patients with breast conservative surgery and positive hormone receptors. In postmenopausal women, treatment with aromatase inhibitors for 5 years can be considered. 6,7

In case of mastectomy, see chemoprevention section.

\section{Follow-up}

Mammary gland evaluation in cases of DCIS treated with conservative surgery should include a mammography after surgical treatment and prior to radiotherapy to verify complete resection of the lesion. In cases where postoperative radiotherapy is not considered, it shall be carried out as soon as the test is considered to be tolerable for the patient. Subsequently, an annual mammography shall be performed. In specialized centers, an interdisciplinary team will 
be able to evaluate, in special situations, the proposal of prophylactic bilateral mastectomy, which has demonstrated to be safe and efficacious at reducing the likelihood of cancer in the future in high-risk asymptomatic women. ${ }^{8}$

\section{Lobular carcinoma in situ (LCIS)}

This is an infrequent lesion and its histological and differential diagnosis with atypical hyperplasia requires the intervention of expert pathologists. Generally, it is not associated with a palpable lump or specific mammographic changes. This lesion is regarded as a risk marker and not a cancer that directly evolves into the invasive form. About $10 \%$ to $15 \%$ of patients will develop an invasive carcinoma in either breast in their lifetime, generally of the infiltrating ductal type. The risk for invasive breast cancer appearance is close to $0.5 \%$ per follow-up year, and when it is associated with first-degree genetic makeup, the risk increases to $1 \%$ per year.

The treatment of choice is excision of the affected zone after verifying there is no clinical, radiological or histological residual or additional lesion. Adjuvant radiotherapy or medical therapy is not indicated. The LCIS pleomorphic subtype has been considered to entail higher risk for the development of invasive disease and this special subgroup might be a lesion that evolves to invasive carcinoma and not only a risk marker. All patients with LCIS should be included in a close follow-up and surveillance program, in addition to receiving counseling with regard to chemoprevention or prophylactic bilateral mastectomy.

\section{Stages I and II workup}

Workup for these cases (except for T3 NO M0) should include:

- Thorough, directed history. Emphasis should be made on family history of breast, ovary, pancreas and colon cancer; risk factors for breast cancer; and careful questioning about symptoms denoting visceral or bone metastases. Physical examination should identify tumor size, location and characteristics, as well as other breast signs, in addition to the presence or absence of adenomegalies in lymph node-bearing regions. Conditions of contralateral breast should be also mentioned.

- Tumor staging with the TNM and pTpNpM system.
- General laboratory tests.

- Postero-anterior chest X-ray.

- Bilateral mammography with cephalo-caudal and lateral-oblique projections.

In women younger than 40 years and in those with highly dense breasts, it can be useful or necessary including breast US or MRI in order to assess multicentrality and bilaterality. Liver or bone metastases should be investigated if there are symptoms suggestive of dissemination or alterations in the liver function tests.

In a suspicious lesion, performing a preoperative biopsy prior to definitive treatment is recommended, with minimal invasion or by marking, so that histological diagnosis is documented.

Core needle (tru-cut) biopsy has the advantage that it enables more complete histological examination with less possibilities of error. Fine needle aspiration biopsy (FNAB) is not recommended.

It is highly important avoiding fragmented excisional biopsies, formation of hematomas, drainage by counteropening and incisions distant to the tumor site. All this complicates subsequent management and reduces the possibility of conservative surgery performance.

\section{Surgical treatment modalities at stages I and II}

Surgical treatment for these stages (except for T3 NO M0) can be as follows: ${ }^{1-3}$

- Conservative treatment: it involves three-dimensional resection of the tumor and surrounding healthy tissue with free margins and treatment of the corresponding axillary region. Its purpose is local control of the primary tumor while preserving breast esthetics.

- Radical treatment: modified radical mastectomy. ${ }^{4-6}$

These options should be offered in simple and objective language to the patient.

\section{A. Conservative treatment}

It includes surgery, radiotherapy and, in most cases, systemic adjuvant therapy. The success of this management is based on optimal patient selection and multidisciplinary participation of the treating team. With this type of surgery, together with postoperative radiotherapy, similar possibilities of survival and locoregional control are offered with regard to radical mastectomy, but with the important advantage of breast preservation. 


\section{Indications}

- Patients at stages I and II with primary tumor $\leq 3 \mathrm{~cm}$ who wish to preserve the breast and accept treatment with radiotherapy after surgery.

- In highly selected cases with tumors $>3 \mathrm{~cm}$, and even $>5 \mathrm{~cm}$ (T3), initiating neoadjuvant systemic treatment is possible to reduce the size of the primary tumor and carry out conservative surgery or surgery with an oncoplastic pattern from the start.

2. Contraindications

- Impossibility to obtain negative margins. Clinical or radiological multicentrality.

- Inability to obtain an adequate cosmetic outcome owing to breast-tumor ratio and location. However, the use of oncoplastic surgery techniques that enable the displacement of fibroglandular tissues with adequate cosmetic results can be considered.

- No radiotherapy available or contraindication to receive it.

- Explicit rejection by the patient.

3. Conditions to perform conservative treatment

- The treatment should be performed by a surgical oncologist with training and experience in breast cancer conservative management.

- Having a qualified pathologist who knows and uses histological prognostic markers.

- Having access to radiotherapy treatment.

If the patient is candidate and accepts neoadjuvant chemotherapy with the purpose to carry out conservative treatment, it is mandatory for original tumor site and size to be delimited. This is made with a metal clip, preferably at the center of the tumor, since response to this chemotherapy can difficult original neoplasm localization. ${ }^{5}$

The surgeon has the responsibility to obtain tumor-free surgical margins, since this is associated with local recurrence low rate. The presence of tumor cells in the surgical margin forces re-excision or mastectomy. ${ }^{7,8}$

\section{Surgical technique}

- Three-dimensional resection of the tumor should be performed with an adequate peripheral margin, attempting to obtain it ink-free. Surgical margins' marking is indispensable for adequate evaluation of resection limits, in addition to radiographic assessment of the specimen with mammography or US, as well as by pathology during the surgical procedure. Mandatorily, axillary surgical treatment should be concomitantly carried out.

- To facilitate the radiation oncologist's work, metal staples should be left delimiting the mammary gland resection area, since the additional radiation dose administration can be more precise this way.
- Adequate cosmetic result for the breast should be sought without compromising cancer treatment.

- There are new oncoplastic surgery techniques that enable resections of larger tumors with appropriate cosmetic results. For this, procedures carried out by experienced surgeons or with the support of plastic surgeons are used, without affecting cancer control, considering as an important aspect the marking prior to flap manipulation or rotation.

\section{B. Radical treatment (mastectomy)}

Any woman who undergoes mastectomy should be informed about the possibility of breast reconstruction.

Adequate timing and reconstruction technique should be discussed with the patient and the reconstructing plastic surgeon, which should be part of the multidisciplinary team.

1. Recommendations for mastectomy

- Preference of the patient after receiving complete information on her surgical options.

- Multicentric disease with no possibility of free margins.

- Unfavorable breast-tumor ratio.

- Difficulty for adequate follow-up.

- No possibilities of postoperative radiotherapy administration.

2. Skin-preserving mastectomy

This technique, which requires higher experience, consists in practicing a modified radical mastectomy but preserving the largest amount of skin possible, dissecting the areola-nipple complex in case of terminal lactiferous duct margin positivity. ${ }^{1-3,9}$

Axillary dissection can be performed with separate incisions. These and previous biopsy resection site should be planned and the patient programmed for immediate reconstruction with autologous tissue or prosthetic material. This will result in better cosmetic outcome, cost reduction and lower psychological effect, without cancer control being diminished.

\section{Oncoplastic surgery}

Breast oncoplastic surgery is an approach to conservative treatment that enables tumor wide excision without compromising the natural appearance of the mammary gland. It is based on plastic surgery techniques integration for immediate remodeling of the breast after cancer wide excision, since conservative surgery success is based on complete tumor extirpation, with adequate margins, but preserving natural appearance. 
The limiting factor is the amount if removed tissue, not only in absolute terms, but in relation to tumor location and breast size. ${ }^{1}$

Clough et al. propose classifying oncoplastic techniques in two groups:

a) When the volume of tissue to be excised is lower than $20 \%$. These techniques can be applied by a surgical oncologist with no specific training in plastic surgery.

b) When $20 \%$ to $50 \%$ of breast volume is foreseen to be excised and dissecting skin excess is required to remodel the breast. These techniques are based on those of mammoplasty and require specific training in oncoplastic surgery, since contralateral breast symmetrization has also to be performed in a simultaneous or deferred form.

These oncoplastic procedures have enabled to broaden breast-preserving treatment indications to patients who had to undergo mastectomy owing to the impossibility to obtain adequate esthetic results after tumor excision. ${ }^{2-6}$ Optimal results are obtained in the setting of multidisciplinary teams that include surgeons credited in breast surgery and reconstructive surgery, focused not only on achieving adequate oncologic results, but on esthetic outcomes, in line with the patient's desires to achieve an optimal quality of life. ${ }^{7-9}$

In oncoplastic surgery, the tumor bed should be marked with staples after resection and prior to reconstruction in order to facilitate for the radiation oncologist to identify the area that is to receive additional dose. ${ }^{8-10}$

\section{Surgical treatment of the axilla}

Patients with clinical stage I and II invasive breast cancer require histopathological evaluation of the lymph node status. SLN mapping and resection is recommended for surgical staging of clinically negative axilla. ${ }^{1-4}$

This recommendation is based on the results of randomized trials that have demonstrated lower morbidity (pain, lymphedema and sensory loss) both in the shoulder and upper limb in patients with breast cancer undergoing the SLN procedure versus standard axillary dissection..$^{3-6}$ In none of these trials were there documented differences in the procedure effectiveness in comparison with level I and II axillary dissection to determine the presence or absence of metastasis.

With regard to the SLN technique, several studies have demonstrated high consistency regardless of the radioisotope or dye site of injection. ${ }^{7}$ The preferred localization technique is with double marker (radiotracer and dye); however, in sites where a nuclear medicine department is not available, the performance of the SLN procedure with dye has been documented to be a valid technique, with similar identification rates than those obtained with double marker. ${ }^{8,9}$

An experienced team is required for the performance of the SLN procedure. ${ }^{10}$ In case there is no experienced group available, patients with clinical stage I and II invasive breast cancer should be referred to institutions where staging the axilla by means of SLN assessment is possible.

The SLN procedure is also recommended in cases of extended ductal carcinoma in situ that is to be treated with mastectomy, given that performing the SLN procedure is not possible if invasive carcinoma is documented in the surgical specimen.

Not all patients are candidates to a SLN procedure. The procedure requires a clinically negative axilla or else corroboration (by means of core needle or fine needle aspiration biopsy) that suspicious lymph nodes are negative for metastatic disease. Following neoadjuvant chemotherapy, sentinel lymph node biopsy is recommended if axillary lymph nodes that were initially regarded as being clinically negative remain negative after chemotherapy. In a clinically positive axilla after neoadjuvant treatment, axillary lymphadenectomy will be performed regardless of treatment response.

After SLN excision, if the patient has a T1 or T2 tumor, 1 or 2 positive SLN, has not been treated with neoadjuvant chemotherapy and is to undergo conservative surgery with adjuvant radiotherapy, axillary dissection is not recommended. ${ }^{11}$

The AMAROS trial concludes that axillary dissection and radiotherapy to the axilla in the presence of a positive SLN provide an excellent control that is comparable to that of T1 and T2 tumors with non-palpable lymph nodes, with less radiotherapy-associated morbidity. ${ }^{12-14}$ In case there is sentinel lymph node capsular rupture or extracapsular invasion, complementary treatment to the axilla is recommended with surgery or radiotherapy.

Level I and II axillary dissection is recommended for the treatment of patients with clinically or cytologically positive lymph nodes. In case of having the resources, US-guided cytological confirmation is recommended in patients with clinically positive lymph nodes. If negative, the patient is candidate to lymphatic mapping with SLN. If axillary disease is documented prior to neoadjuvant chemotherapy by any method (aspiration biopsy, SLN), axillary dissection is recommended at treatment conclusion. 
In traditional level I and II axillary dissection, assessment of at least 10 lymph nodes is recommended for correct axillary staging. In case of having less than 10 lymph nodes, management is individualized according to patient characteristics. Level III lymph node dissection is only indicated if there is macroscopic disease at this level.

\section{E. Breast reconstruction}

Breast reconstruction should be offered to all patients that are to undergo mastectomy. It can be performed in an immediate or late form.

The advantages of immediate reconstruction are:

a) Great psychological benefit.

b) One less surgical procedure.

c) Less fibrosis formation and cicatricial retraction.

Available methods for breast reconstruction

a) Reconstruction with alloplastic materials.

b) Reconstruction with autologous tissues.

c) Combination of both methods.

The method to choose the type of breast reconstruction should contemplate:

a) Type of mastectomy.

b) Adjuvant therapy (if the patient is to receive or has already received radiotherapy).

c) Available tissues for reconstruction.

d)Contralateral breast size and shape.

e) Presence or not of associated conditions.

f) Patient expectations.

g)Familiarity with the different reconstruction techniques.

Patients should consider:

a) Desired technique (some ask for a known technique).

b) Number of procedures each surgical technique entails.

c) Scars resulting from the procedure.

d) If she wants for contralateral breast to be modified or not.

e) Desire or rejection to the use of implants.

f) Risks and benefits of each surgery.

g) Cost of each procedure.

\section{BREAST RECONSTRUCTION TECHNIQUES}

Breast reconstruction with expander followed by implant $^{1}$

Indications:

- Sufficient skin in quantity and of good quality.

- Pectoris major muscle preservation.
Complications:

I) Expander:

- Infection.

- Necrosis/exposure.

- Dehiscence.

- Seroma.

- Hematoma.

II)Implant;

- Capsular contracture.

- Displacement.

- Asymmetry.

- Less naturalness.

Breast reconstruction with autologous tissues (flaps) ${ }^{2}$ Indications:

- Insufficient skin for expansion.

- Skin with radiotherapy-related damage.

- Absence of pectoralis major muscle.

- Infraclavicular depression.

- Implant rejection.

Advantages of breast reconstruction with autologous tissue vs. alloplastic materials ${ }^{3-5}$

Advantages:

- Better long-term esthetic results.

- Reconstruction with more natural appearance.

- Almost identical consistency to that of normal breast.

Disadvantages:

- Longer surgical time.

- Longer time for recovery.

- Donor site complications.

Reconstruction options with autologous tissue

a) Extended latissimus dorsi flap.

b) Pediculated TRAM flap and abdominal free flaps.

c) Non-TRAM free flaps.

Breast reconstruction with extended latissimus flap ${ }^{6}$

Advantages:

- Flap reliability.

- Color and texture quite similar to those of the breast.

Disadvantages:

- An implant is required to provide volume in up to $90 \%$ of cases.

- Hypertrophic scar in donor area.

- High rate of seroma formation.

Breast reconstruction with TRAM flap ${ }^{7-9}$

Vascular options:

- Pedicled in a single muscle.

- Pedicled with both muscles.

- Supercharged.

- Delayed. Free. 
Abdominal free flap options ${ }^{10,11}$

- Perforator (DIEP).

- Muscle preserving.

- Based on the superficial inferior epigastric artery (SIEA).

Advantages of abdominal free flaps vs. pedicled TRAM $^{12,13}$

1. Higher blood flow.

2. Lower incidence of fat necrosis.

3. Less coloration changes and atrophy.

4. Higher versatility for remodeling.

5. Less amount of muscle.

6. Less morbidity at donor area.

7. No epigastric bulging.

Breast reconstruction with non-TRAM free flaps ${ }^{14-16}$

- Superior gluteus.

- Inferior gluteus.

- Transverse gracilis (inner thigh).

These flaps are indicated when the TRAM flap is not available due to previous dermolipectomy.

Radiotherapy and breast reconstruction

Breast reconstruction with autologous tissues is not contraindicated in irradiated patients or with postoperative radiotherapy indication, since the cosmetic result and the risk for complications are equivalent. ${ }^{17-19}$

Radiotherapy-associated complications

Healing problems

- Fibrosis.

- Flap necrosis.

- Capsular contractures.

- Implant displacement.

- Breast volume reduction.

- Poor symmetry and projection.

\section{Conclusions}

Reconstructive surgery plays a highly important role in the breast cancer patient treated with mastectomy or conservative surgery.

Multidisciplinary management enables cancer control possibilities optimization in close coordination of treatment modalities, including radiotherapy, systemic treatment and surgery. With appropriate knowledge of all these aspects, the reconstructing surgeon will be able to offer each patient individualized options to satisfy her expectations.

\section{F. Risk-reducing mastectomy}

The practice of this type of mastectomy has increased in part owing to an overestimation of the risk for breast cancer both by doctors and patients. It is an option to be considered when the risk for the development of breast cancer is elevated. Since only few patients will have an overall survival benefit, a multidisciplinary discussion is recommended to determine individual risk in addition to prevention alternatives. The discussion can be appropriate upon patient request and in any of the following situations: ${ }^{1-3}$

- Early age of onset.

- Family history of breast cancer suggesting a hereditary pattern.

- Low probability of regular follow-up.

The patient shall be informed on the risks and benefits, as well as about the fact that the procedure does not offer absolute protection against breast cancer and has implications on body image and secondary psychosexual effects. ${ }^{3}$

If a risk-reducing mastectomy is performed, the anatomical limits of a therapeutic mastectomy with skin and areola-nipple complex preservation have to be used, with no need for axillary staging. 4.5

\section{Potential indications for risk-reducing mastectomy without a cancer diagnosis}

1. Evident family history of cancer without demonstrable genetic susceptibility, such as:

- Young age at cancer onset ( $<40$ years).

- Two breast or ovary/fallopian tube/peritoneum primary cancers in first-degree relatives or one in a first-degree with two in second-degree relatives.

- Combination of breast cancer with one or more of the following: thyroid or pancreas cancer, brain tumors, diffuse gastric cancer, skin manifestations of leukemia/lymphoma in the same family branch.

- Relatives with known breast cancer mutations.

- At-risk population (Ashkenazi Jewish females of any age with breast or ovarian cancer).

- History of male breast cancer.

- Ovarian/fallopian tubes/peritoneum cancer.

2. Mutation of BRCA 1 and 2 susceptibility genes.

3. Other mutations less commonly associated with breast cancer, such as mutations in the TP53 and PTEN genes (linked with Li-Fraumeni and Cowden syndromes).

4. Histological risk factors (e.g., lobular neoplasia in situ, lobular and ductal atypical hyperplasia).

Potential indications for contralateralo risk-reduction mastectomy (patients with current or previous breast cancer diagnosis)

1. Risk reduction.

2. Esthetic and reconstructive issues (asymmetry, balance). 
3. Difficulty for surveillance (breast density, diffuse microcalcifications).

4. Contralateral breast biopsy with histological result of lobular neoplasm in situ or lobular or ductal atypical hyperplasia.

\section{Adjuvant systemic therapy at operable stages}

With the purpose to establish optimal adjuvant therapy, the clinical oncologist must have complete information on tumor characteristics. In particular, expression or not of therapeutic targets (hormone receptors and HER-2 neu) has significant importance to offering the best individualized treatment.

\section{Definition, indications and objectives}

Adjuvancy refers to any antineoplastic treatment administered after surgical management; its goals are to prolong the disease-free period, reduce local and systemic recurrence and increase overall survival. ${ }^{1-3}$ Systemic adjuvant treatment (hormone therapy \pm chemotherapy \pm trastuzumab) should be assessed and administered by a medical oncologist owing to the updating degree required and to complications and toxicities that can relate to it.

Patients with positive lymph nodes. Owing to the high risk for relapse in this group, all patients with positive lymph nodes should receive adjuvant systemic treatment (chemotherapy \pm hormone therapy \pm trastuzumab), regardless of the number of lymph nodes involved.

Patients with negative lymph nodes. Adjuvant systemic treatment (chemotherapy \pm hormone therapy \pm trastuzumab) administration is recommended when there is any of the following conditions: ${ }^{4,5}$

- Tumor $>1 \mathrm{~cm}$ (more than $3 \mathrm{~cm}$ for favorable histology such as tubular and mucinous cancer) with positive hormone receptors and negative HER-2 (hormone therapy \pm chemotherapy).

- Triple-negative tumor $>5 \mathrm{~mm}$ (chemotherapy).

- Tumor > $5 \mathrm{~mm}$ with HRR-2 neu oncogene overexpression (chemotherapy \pm trastuzumab \pm hormone therapy).

- Oncotype DX with high recurrence score $(\geq 31)$ in cases where it is available (chemotherapy \pm hormone therapy).

Consider also systemic treatment (chemotherapy \pm hormone therapy \pm trastuzumab) if any of the following characteristics is present:
- High grade tumor.

- Presence of lymphovascular invasion.

- Oncotype CX with intermediate recurrence score (18-30).

- Age $<35$ years.

\section{Selection of adjuvant systemic therapy}

Systemic therapy should be initiated as soon as possible, preferably prior to 6 weeks after surgical treatment. Simultaneous use of radiotherapy and chemotherapy is not recommended owing to toxicity increase. When both are indicated, treatment should be started with chemotherapy and at its conclusion radiotherapy will be applied. Concomitant chemotherapy and hormone therapy is also not suggested; the latter should be started until the conclusion of the former.

Retrospective studies have consistently demonstrated that delays in adjuvant chemotherapy administration are related to overall survival and breast cancer-associated survival decrease. Patients in whom adjuvant chemotherapy administration is delayed have worse prognosis regardless of tumor subtype, although the impact appears to be greater on those with highly proliferative tumors, such as triple-negative lesions and those overexpressing HER-2 neu. It should be noted that, in various studies, delays in adjuvant chemotherapy administration are more common in older patients, with more comorbidities and with socio-demographic disadvantages. ${ }^{6}$

\section{A. Adjuvant treatment with chemotherapy}

\section{General guidelines}

- Chemotherapy should be indicated and duly supervised by a medical oncologist, in an adequate physical area and aided by a nurse specialized in oncology and neoplastic drugs administration. The necessary anti-emetics should be available to reduce gastrointestinal toxicity, as well as colony-stimulation factors to prevent or treat neutropenia.

- The use of anthracycline-based regimens is recommended owing to the modest benefit in disease-free survival and overall survival when compared with previous regimens such as $\mathrm{CMF}^{1-3}$ In addition, taxane administration has demonstrated moderate benefit that is independent of hormone receptors expression, number of axillary lymph nodes involved or menstrual status. $3,7,8$ 
- In patients with triple-negative tumors, using the aforementioned regimens is recommended, since up to this moment there is no evidence to indicate other regimens or drugs.

The strongest evidence of benefit for adjuvant chemotherapy is obtained with third generation regimens:

- FAC or FEC followed by weekly paclitaxel. ${ }^{9,10}$

- FEC-100 followed by docetaxel every three weeks. ${ }^{11}$

- EC/AC followed by weekly paclitaxel.12,13

- TAC. $^{14}$

- TC. $^{15}$

- Dose-dense AC followed by dose-dense paclitaxel. ${ }^{16}$

- Dose-dense AC followed by weekly paclitaxel. ${ }^{16}$

Dose-dense chemotherapy regimens with AC every two weeks followed by weekly paclitaxel plus filgrastrim achieve a $26 \%$ reduction in the risk for recurrence and of $31 \%$ in the likelihood of death. ${ }^{16}$

With regard to the administration sequence between anthracyclines and taxanes, a recently published meta-analysis supports the use of taxanes followed by anthracyclines as a reasonable option in everyday clinical practice. The results obtained in terms of pathological responses in some phase III clinical trials support this suggestion as well.

The inclusion of other drugs such as gemcitabine, platinum salts or capecitabine to anthracycline and taxane-based regimens is not recommended in the adjuvant setting, since studies in neoadjuvancy have not demonstrated clinical benefit.

\section{B. Adjuvant treatment with hormone therapy}

\section{Carcinoma in situ}

- Tamoxifen ( $20 \mathrm{mg} /$ day) for 5 years is recommended as relapse risk-reducing therapy is recommended in patients with breast-preserving surgery and positive hormone receptors. ${ }^{1-3}$ For postmenopausal women, treatment with an aromatase inhibitor for 5 years can be considered. ${ }^{4,5}$

- In case of mastectomy, see chemoprevention section.

Invasive carcinoma

Premenopausal patients* at diagnosis

- Tamoxifen ( $20 \mathrm{mg} /$ day) for 5 to 10 -year duration is recommended in premenopausal or perimenopausal women with positive or unknown hormone receptors. ${ }^{3}$ For women that after 5 years of treatment with tamoxifen are considered to be postmenopausal, extended therapy with aromatase inhibitors for 5 additional years is recommended.

- For women that are still premenopausal after having received chemotherapy (or have recovered ovarian function 8 months after chemotherapy conclusion) and with any high-risk factor (younger than 35 years, tumors larger than $2 \mathrm{~cm}$, positive lymph nodes and histological grade 3), treatment with aromatase inhibitors or tamoxifen for 5 years plus ovarian ablation (medical, radiotherapeutic or surgical ablation) is recommended. 6,7

- Starting with medical ablation is advised to assess tolerance and adverse effects prior to recommending a permanent ablation method.

Postmenopausal patients* at diagnosis.

Aromatase inhibitors for 5 years are recommended in postmenopausal women with positive hormone receptors.

- In patients in whom therapy with tamoxifen is initiated for 2 to 3 years and continuing with an aromatase inhibitor is decided, administering it for 2 to 5 years is recommended. 8,9

- For patients started on therapy with tamoxifen for 5 years, 5 additional years with an aromatase inhibitor can be considered, or with tamoxifen in case of intolerance, contraindication or lack of access to aromatase inhibitors.

- Prior to considering the prescription of extended therapy (for more than 5 years) it is important for life expectancy, presence of high-risk clinicopathological factors (e.g. positive lymph nodes, grade, size, etc.), previous treatment tolerance and each patient's comorbodities to be evaluated.

*Menopause definition: patients with bilateral oophorectomy, age $\geq 60$ years, age $\leq 60$ years and amenorrhea for 12 months or more in the absence of chemotherapy, tamoxifen, toremifene or ovarian suppression and follicle-stimulating hormone (FSH) and estradiol levels at postmenopausal ranges. In case of being on treatment with tamoxifen and being $\leq$ 60 years of age, FSH and estradiol serum levels at menopausal ranges are necessary. In women that at chemotherapy initiation are premenopausal, amenorrhea is not a menopausal status indicator, and carrying out serial measurements of these hormones is therefore recommended prior to the aromatase inhibitors indication. ${ }^{10}$

\section{EXTENDED ADJUVANT HORMONE THERAPY}

Five years of tamoxifen plus 5 years of aromatase inhibitors or continuing tamoxifen for 5 additional years 
is an option for women with poor prognosis, especially in patients with positive lymph node disease. ${ }^{11,12}$

\section{Adjuvant treatment with targeted therapies (trastuzumab)}

In patients with tumors with HER-2 neu IHC +++ or $\mathrm{FISH}+$ overexpression, the use of the monoclonal antibody trastuzumab in combination with adjuvant chemotherapy has allowed for benefit to be obtained both in relapse-free survival (HR 0.62) and overall survival (HR 0.66). ${ }^{1-3}$

Starting adjuvant treatment early with trastuzumab together with taxane-based chemotherapy followed by anthracycline is recommended, since this sequence has achieved better outcomes. ${ }^{4}$ Trastuzumab and anthracycline simultaneous administration is advised against given that it increases cardiotoxicity.

The TCH regimen (docetaxel, carboplatin and trastuzumab) for 6 cycles without the use of anthracyclines should be considered in patients at high-risk for cardiovascular disease (previous history or heart failure, older age, high blood pressure or previous anthracycline use). 5,6

Currently, the duration of adjuvant treatment with trastuzumab is recommended to be 1 year, since administration for less or more time have not demonstrated better results. ${ }^{6-9}$

In selected cases with negative lymph nodes and small tumors (less than $3 \mathrm{~cm}$ ), the weekly paclitaxel + trastuzumab regimen for 12 weeks followed by trastuzumab every 3 weeks until 1 year is completed can be an option. ${ }^{10}$

Patients receiving trastuzumab should be carefully assessed owing to the risk for cardiotoxicity, especially those with a personal history of heart disease or at high risk. Left ventricle ejection fraction (LVEF) should be assessed prior to starting this agent, every 12 weeks and at treatment completion. All patients receiving this drug should be monitored with echocardiography or nuclear gammagraphy in order to early detect ventricular function decrease (Table 1).

Adjuvant treatment with other targeted therapies is currently not indicated.

\section{Breast cancer medical treatment-derived mid and long-term toxicity}

Early diagnosis and new therapeutic advances implementation have improved the prognosis of patients with early breast cancer and significantly increased the
Table 1. Behavior to be followed for cardiologic surveillance and drug dose adjustment.

\begin{tabular}{|c|c|c|c|}
\hline & \multicolumn{3}{|c|}{ LVEF absolute decrease } \\
\hline & $<10 \%$ & $10-15 \%$ & $>15 \%$ \\
\hline Normal LVEF & Continue & Continue & Discontinue* \\
\hline $\begin{array}{l}1-5 \% \text { below } \\
\text { LVEF NL }\end{array}$ & Continue & Discontinue* & Discontinue* \\
\hline $\begin{array}{l}>5 \% \text { below } \\
\text { LVEF NL }\end{array}$ & Discontinue* & Discontinue* & Discontinue* \\
\hline
\end{tabular}

LVEF: Left ventricle ejection fraction; NL: Normal limit. ${ }^{*}$ Repeat LVEF in 4 weeks.

number of survivors. Hence, knowing medical treatment-derived toxicities and being familiarized with their recommended management is essential, given the huge impact they produce on patients' quality of life. ${ }^{1}$

\section{A. Cardiotoxicity}

\section{Anthracyclines}

Cardiotoxicity related to the use of adriamycin or epirubicin occurs as an asymptomatic systolic dysfunction, with left ventricular ejection fraction (LVEF) up to more than $15 \%$ decrease when doxorubicin cumulative doses higher than $240 \mathrm{mg} / \mathrm{m}^{2}$ are used. The risk for the development of cardiotoxicity with epirubicin is $1 \%$ with cumulative doses of $550 \mathrm{mg} / \mathrm{m}^{2}, 1.5 \%$ with cumulative doses of $700 \mathrm{mg} / \mathrm{m}^{2}$; the risk increases significantly with higher doses, and doses higher than $900 \mathrm{mg} / \mathrm{m}^{2}$ are therefore not recommended. A small percentage of patients can experience heart failure, which increases with cumulative dose and is generally not reversible. ${ }^{2}$ Associated risk factors are:

- Age older than 65 years.

- History of high blood pressure or cardiac comorbidities.

- High cumulative doses (1\% risk with doses of $240 \mathrm{mg} / \mathrm{m}^{2}, 5 \%$ with $400 \mathrm{mg} / \mathrm{m}^{2}$, and a dramatic increase from $550 \mathrm{mg} / \mathrm{m}^{2}$ on with adriamycin).

- History of radiation to the mediastinum.

- Combination with trastuzumab.

Recommendations:

- Perform baseline echocardiogram or multigated acquisition (MUGA) scan in patients older than 50 years or in younger women with heart comorbidities.

- Do not exceed the dose (the risk is low with $A C x$ 4, FAC $\times 4$, EC $\times 4$ or FEC $\times 4$ ). 
- Clinical monitoring of symptoms and, given the case, opportune referral to cardiology.

\section{TRASTUZUMAB}

Trastuzumab-related cardiotoxicity is generally reversible and is associated with the damage caused by the anti-HER-2 blockage at the level of cardiac myocytes. Heart failure incidence ranges from $1.5 \%$ to $5 \%$, but that of asymptomatic LVEF decrease is $4 \%$ to $20 \%$. Risk factors are unclear; however, older patients, with baseline LVEF of $50 \%$ to $54 \%$, cardiac comorbidities and use of anti-hypertensive drugs are known to be at higher risk. ${ }^{3,4}$ The risk for cardiotoxicity can be higher in patients treated with sequential anthracyclines. Management with beta-blockers and angiotensin-converting enzyme inhibitors improves LVEF and, in many cases, heart function can be normalized. In selected patients, reinitiating trastuzumab treatment is possible, but this should only be done in patients who are managed together with a cardiologist.

\section{Recommendations}

- Echocardiogram or MUGA scan prior to treatment start and every 3 months until its completion (months $0,3,6,9$ and 12).

- If there is LVEF decrease, discontinue trastuzumab and treat heart failure.

- If LVEF improves, resuming the treatment is possible under close supervision by cardiology.

\section{B. Leukemia and myelodysplastic syndrome}

Acute myelocytic leukemia and myelodysplastic syndrome have been associated with the use of alkylating agents and occur between 5 and 7 years after treatment. ${ }^{5}$ Increased risk for hematological malignancies secondary to topoisomerase inhibitors administration, including anthracyclines, has also been reported, and they usually occur 3 to 5 years after their use. The risk associated with the use of taxanes is not well characterized given the relatively recent introduction of this type of drugs.

The 5 -year cumulative rate is $0.24 \%$, but it rises to $0.48 \% 10$ years after treatment conclusion. In comparison with patients treated only with surgery, those who receive chemotherapy have a 6.8-fold higher risk and the risk increases to 7.6-fold if they are treated with chemotherapy and radiotherapy. However, it is important to remember that the absolute number of patients who develop a secondary hematological malignancy is small, with a rate of $0.46 / 100$ person-years in patients treated with chemotherapy. ${ }^{6}$

\section{Neuropathy}

Neuropathy is a highly common complication in patients who receive treatment with taxanes. The incidence ranges from $13 \%$ to $27 \%$, and varies according to the type and frequency of the taxane used. ${ }^{7}$ In severe cases, this complication can even to be disabling and permanent. Factors associated with this toxicity include advanced age, ethnicity, obesity, diabetes mellitus and history of alcohol abuse. To date, there is no efficacious preventive method and therapeutic options have limited benefit. ${ }^{8,9}$

\section{TREATMENT:}

- Duloxetine.

- Gabapentin, pregabalin: limited benefit in clinical trials; their effect appears at high doses and after weeks to months of treatment. Their administration is limited by the somnolence and tiredness they cause.

- Opioids in severe cases.

- Antidepressants: nortriptyline, venlafaxine and fluoxetine have shown effects in the management of diabetic neuropathy and postherpetic neuralgia. There are no data on patients with neuropathy associated with the use of taxanes.

- Acupuncture.

- Relaxation therapy.

- Occupational therapy.

- Electrical neurostimulation.

- Massage.

\section{Fatigue}

This is the term used to define a persistent tiredness sensation not proportionally associated with physical activities. It occurs in up to $80 \%$ of chemotherapy-treated patients and persists for 6 to 12 months after treatment finalization in $30 \%$ of cases. Unfortunately, therapeutic strategies are limited, with symptom improvements occurring slowly. Evidence has demonstrated that increasing physical activity is the most efficacious strategy to improve fatigue. ${ }^{10}$

\section{Recommendations}

- Assess for the presence of fatigue at regular intervals. 
- If fatigue is moderate to severe, rule out other causes (disease recurrence, wakefulness-sleep disturbances, depression, anxiety, pain, nutritional anomalies, hypothyroidism, vitamin $\mathrm{D}$ deficiency, etc) and treat accordingly.

Interventions:

- Physical activity increase (150 min moderate aerobic exercise per week and 2 to 3 strength training sessions).

- For patients who are not in conditions to exercise, walking is recommended or, at least, physical therapy.

- Cognitive and psychosocial interventions: relaxation techniques, support groups, etc.

- Body-mind interventions: yoga, acupuncture, massage.

- Pharmacological interventions: this type of strategies should be considered only if all previously mentioned alternatives have been evaluated. Modafinil or methylphenidate can be used; randomized trials have demonstrated little efficacy in patients with breast cancer, but there can be improvement in severe fatigue cases. Evidence suggests that symptom improvement is common when modafinil is used during treatment, with limited efficacy in patients who have completed therapy. ${ }^{11,12}$

\section{E. Cognitive dysfunction}

The causes of this complex toxicity that occurs on the mid and long-term are so far unclear. The incidence of cognitive harm secondary to chemotherapy is $20 \%$ to $30 \%$. There are reports indicating that $17 \%$ to $75 \%$ of women suffer cognitive changes owing to the instituted treatment and probably also due to the impact caused by diagnosis. Currently, there are no proven interventions for the prevention or management of breast cancer diagnosis and treatment-related cognitive alterations; neither do international guidelines propose specific norms. ${ }^{13}$ In patients with persistent cognitive deterioration, neurocognitive evaluation is essential.

\section{F. Medical treatment-induced menopausal symptoms}

The prevalence of chemotherapy and hormone therapy-induced climacteric symptoms (hot flashes and night sweats, vaginal dryness and atrophy, incontinence, dyspareunia, insomnia, irritability, joint pain, fatigue) varies according to the age, type of treatment and number of administered chemotherapy cycles. These symptoms get to occur in more than $40 \%$ of patients.

Since hormone replacement therapy is contraindicated, multiple drugs have been used for pharmacological treatment with generally unsatisfactory results.

\section{Recommendations: ${ }^{14}$ \\ - Physical exercise. \\ - Paused breathing. \\ - Muscle relaxation, meditation, yoga. \\ - Cognitive-behavioral therapy. \\ - Behavioral interventions combination. \\ - Hypnosis. \\ - Acupuncture. \\ - Venlafaxine.}

\section{G. Chemotherapy-induced ovarian failure}

All patient at childbearing age should receive counseling on the probable loss of ovarian function and be referred to a oncofertility specialist if possible. There are important advances in this field: there are already clinics in this area that propose cryopreservation or ovarian stimulation or preservation protocols wit good safety margins. There is evidence that goserelin simultaneously administered with chemotherapy in patients with hormone receptor-negative tumors helps to preserve ovarian function. A more detailed review of this subject can be found in the section on breast cancer in younger women.

In breast cancer survivors, limited evidence suggests that pregnancy after treatment does not increase recurrence rates and neither compromises the baby's health. Patients who wish to get pregnant are advised to do it 2 to 3 years after chemotherapy completion. All should receive close counseling from their oncologist and their gynecologist. ${ }^{15}$

\section{Adjuvant radiotherapy}

\section{Postoperative radiotherapy in conservative management}

Patients treated with conservative surgery should receive external beam radiotherapy to the breast with two tangential fields. The dose shall be from 45 to 50.4 Gy either with photons, electron beam or brachytherapy, according to radiotherapy equipment availability and radiation oncologist experience. 
It should be insisted that the surgeon should leave radio-opaque references at the surgical margins in order to facilitate more precision in additional dose administration. ${ }^{1}$

\section{Hypofractionation}

Hypofractionation (higher dose per fraction, lower number of fractions and less treatment total time) has the following indications: conservative surgery, patients $\geq 50$ years, $\mathrm{pT} 1-\mathrm{T} 2 \mathrm{~N} 0$ tumor, negative margins. ${ }^{2}$

Hypofractionation requires 3D planning. Within the breast, along the central axis, minimum dose should not be lower than $93 \%$, and maximum dose should not be higher than $107 \%( \pm 7 \%)$ with regard to the prescribed dose. Post-mastectomy hypofractionated radiotherapy or to lymph node areas is not indicated. ${ }^{3}$

Hypofractionation does not reduce locoregional control or worsen long-term cosmetic result; it can decrease acute toxicity in comparison with the standard scheme. $^{4}$

\section{Accelerated partial breast irradiation}

Another conservative treatment alternative for T1-2 $(<3 \mathrm{~cm})$ N0 M0 stage is accelerated partial breast irradiation. Indicated recommendations are: postmenopausal patients, no BRCA 1 and 2 mutation, tumor size $T$ $<2 \mathrm{~cm}$, negative surgical margin, positive hormone receptors, no multicentrality or multifocality, negative lymph nodes.

There are multiple radiotherapy modalities for this approach: brachytherapy, intra-operative radiotherapy and intensity-modulated radiotherapy (IMRT). The results in terms of local recurrence with 5-year follow-up in randomized trials demonstrate non-inferiority, with no differences in toxicity. Cardiopathic patients with left breast cancer who meet the above-mentioned criteria obtain the highest benefit because the dose to the heart is lower. ${ }^{5-7}$

\section{Radiotherapy administration timing}

Radiotherapy initiation after conservative surgery without adjuvant chemotherapy should be in the first 8 weeks, after neoadjuvant chemotherapy and surgery in 30 days, and after surgery and adjuvant chemotherapy in the first month (do not delay more than 7 months from surgery). ${ }^{8-10}$

\section{Indications for adjuvant radiotherapy to lymph node chains}

Axillary-supraclavicular. Either as part of conservative management or of modified radical mastectomy, patients should receive radiotherapy at all axillary levels in case of invasion to four or 4 lymph nodes. All patients in the 1-to-3 positive lymph nodes post-SLN group without lymph node dissection should be irradiated, except if there are micrometastases or isolated cells.

The Mexican Consensus recommends lymph node radiotherapy with 1 to 3 positive axillary lymph nodes after optimal axillary dissection in patients younger than 40 years and with capsular rupture. Other indications include the presence of two or more of the following factors: premenopause, negative hormone receptors, lymphovascular invasion, high-grade tumors (SBR III) and initial $T \geq 2 \mathrm{~cm}$. It is mandatory to use the technique that produces lower toxicity at the level of the brachial plexus. ${ }^{11-16}$

Internal mammary chain. Indications include positive lymph nodes detected by clinical examination or by imaging and inflammatory cancer. The benefit is limited and potential cardiotoxicity should be evaluated in each patient according to already known risk factors for recurrence. ${ }^{17-19}$

\section{Chemotherapy to the chest wall after mastectomy}

Target volume includes the chest wall, the mastectomy scar and drainage orifices are considered.

The chest wall is irradiated in case there is one or several of the following conditions:20,21

- Primary lesion of $5 \mathrm{~cm}$ (T3).

- Skin or pectoralis muscle fascia invasion (T4).

- Positive surgical margin.

- N2.

Lymph node areas shall receive radiotherapy considering the previously mentioned factors.

\section{Radiotherapy associated with} chemotherapy, targeted therapies and hormone therapy ${ }^{21-23}$

The use of radiotherapy concomitant to chemotherapy is not recommended. There is no information contraindicating concomitant administration of radiotherapy with targeted therapies. The concomitant use of hormone therapy 
with radiotherapy has not demonstrated statistically significant increase in pulmonary, cardiac or dermal toxicity.

\section{Neoadjuvant treatment of stage II and III breast cancer, including locally advanced disease}

The term neoadjuvancy refers to an initial or primary systemic treatment that is administered prior to a radical one (surgery or radiotherapy). This group comprises stage III breast carcinomas. However, for the purposes of treatment, certain cases at stages II A/B, T2-3 NO MO and T1-2 $\mathrm{N} 1 \mathrm{M} 0$ are also added. ${ }^{1}$

Initial workup of these patients should include a core or open surgical biopsy of the primary tumor, including a skin segment when deemed useful. Complete histological examination is required including hormone receptors and HER-2 neu status, ${ }^{1}$ in addition to clinical and imaging assessment of the primary tumor and most common potentially metastatic sites by means of chest X-ray or CT, abdominal ultrasound or CT, as well as bone scan (the latter for patients with stages III). PETCT is not indicated as routine test for disease extent.

The therapeutic proposal should be defined by the multidisciplinary medical team and should be based on each patient's characteristics (age, menstrual status, concomitant conditions, preferences, etc.), clinical stage of the disease and primary tumor histological and immunohistochemical variables.

In cases where the disease is technically resectable and breast-preserving surgery is not feasible or wanted by the patient or neoadjuvant chemotherapy effectiveness is expected to be poor (e.g. well-differentiated tumors, mucinous or tubular histology, positive hormone receptors with high titers, HER-2-negative) or its toxicity very high and risky, surgery is recommended as initial procedure. ${ }^{1}$

\section{Neoadjuvant treatment in patients with operable and inoperable stages}

Although the principle of neoadjuvancy was employed in locally advanced stages, this treatment modality is currently used also in patients with tumors initially deemed to be operable, larger than $2 \mathrm{~cm}$ and/or with positive lymph nodes.

The advantages of neoadjuvant therapy are:
1. Increasing the possibilities of conservative surgery.

2. Knowing the pathological response (pCR ypT0/is ypN0) to the treatment, ${ }^{2}$ since this therapy is associated with better prognosis.

If the patient starts with neoadjuvant chemotherapy, marking of the primary tumor site with a radio-opaque clip is recommended for adequate surgical evaluation. ${ }^{3}$

\section{Neoadjuvant chemotherapy}

Standard chemotherapy is considered to be 3 to 4 cycles with anthracyclines followed by 3 to 4 cycles of taxanes (plus trastuzumab in HER-2 neu-positive tumors) prior to surgery, since it is associated with higher odds of $\mathrm{pCR}{ }^{4}$

On the other hand, it is important knowing that the likelihood to attain a pCR after optimal neoadjuvant therapy varies according to the subgroup: hormone-sensitive/HER-2-negative 7\%, triple-negative 30\% and HER-2-positive $32 \%$ to $67 \%{ }^{5}$

Addition of carboplatin to the anthracyclin and taxane regimen in women with triple-negative tumors improves the $\mathrm{pCR}$ rate, and it is therefore considered an option in this patient subgroup; however, this regimen has been associated with higher hematological and non-hematological toxicity. ${ }^{6,7}$ The addition of other drugs such as gemcitabine, capecitabine and nab-paclitaxel is not indicated. ${ }^{8-11}$

\section{Targeted therapies in neoadjuvancy}

The addition of trastuzumab to neoadjuvant chemotherapy in HER-2 neu-positive disease increases pCR, with ranges from $32 \%$ to $67 \%$. Therefore, in patients with HER-2-positive breast cancer, neoadjuvant trastuzumab concomitant administration with taxanes is recommended, ${ }^{12-14}$ but not with anthracyclines. ${ }^{15}$

With regard to dual HER-2 blockade, dual HER-2 blockade therapy with lapatinib, ${ }^{16}$ neratinib ${ }^{17}$ or TDM-118 is not recommended.

However, dual HER-2 blockade with trastuzumab and pertuzumab is associated with significant $\mathrm{pCR}$ increase, and its use is therefore recommended with the following regimens: FEC-THP, TCH-P, AC-THP. ${ }^{19,20}$

Bevacizumab has no current indication in neoadjuvant therapy. ${ }^{21}$

\section{Neoadjuvant hormone therapy}

Neoadjuvant hormone therapy is recommended in postmenopausal women with positive hormone receptors and stages II-III or in patients in whom 
chemotherapy-related toxicity is inacceptable or who have multiple comorbidities. The goal is to increase the likelihood of tumor resection and/or conservative surgery.

Using an $\mathrm{Al}$ is recommended. ${ }^{22,23}$ After hormone therapy is started, if an objective response is obtained, continuing the treatment for at least 4 to 6 months is recommended, ${ }^{24}$ followed by local surgical treatment. Continuing with hormone therapy or chemotherapy will be evaluated according to the pathological response and patient conditions.

\section{Response assessment during neoadjuvant treatment}

Clinical response should be assessed after each chemotherapy cycle, and after the administration of 3 to 4 cycles, assessment of clinical and radiological treatment response (with mammography and/or ultrasound) is recommended. If there is objective response, neoadjuvant treatment shall be continued until completion.

On the other hand, if there is no response, or if data consistent with progression are observed, the following actions can be taken depending on tumor resectability: 1. Consider change of chemotherapy regimen (taxanes $\leftrightarrow$ anthracyclines) for 2 to 4 additional cycles. Subsequently:

a) If operable, perform radical surgery and administer adjuvant radiotherapy.

b) If not operable, radiotherapy treatment can be employed.

- If response is obtained and it can be resected, surgery should be performed.

- If there is no response, second-line chemotherapy should be administered.

\section{Treatment after neoadjuvancy}

According to the response, and once neoadjuvant chemotherapy is concluded, one of the following options can be resorted to:

1. Complete/partial clinical response: the possibility of conservative treatment shall be evaluated; conservative surgery guidelines are similar as in cases with primary surgical treatment. If not eligible or wanted by the patient, modified radical mastectomy should be carried out.

2. In case of stable disease, if the tumor is resectable, surgical treatment should be carried out; if that is not the case, radiotherapy to the breast and lymph node-bearing areas shall be administered. Depending on the response, surgical treatment after radiotherapy, or to continue with second-line systemic treatment, including hormone therapy or targeted therapies if indicated, should be assessed.

3. In patients with positive hormone receptors, hormone therapy will be indicated for at least 5 years, and in HER2 neu-positive tumors, tratsuzumab should be continued until 1 year is completed.

4. Continuing with adjuvant chemotherapy is not indicated if the patient received neoadjuvant anthracyclines and taxanes complete doses, regardless of the obtained response. With regard to radiotherapy, it is advised that all patients with locally advanced disease should receive it. ${ }^{1-3}$

\section{Inflammatory breast cancer}

Inflammatory breast cancer should be treated with neoadjuvant chemotherapy (plus trastuzumab if HER-2-positive). Based on systemic treatment response, locoregional management with modified radical mastectomy and postoperative radiotherapy should be evaluated. If the response to neoadjuvant chemotherapy is poor and the tumor is not resectable, radiotherapy administration followed by radical surgery can be evaluated. ${ }^{4}$

\section{Surgery in locally advanced disease}

Complete locoregional treatment, where extirpation and control of disease is achieved, is associated with better survival. Therefore, surgery and radiotherapy are fundamental in the treatment of locally advanced breast cancer. On the other hand, systemic neoadjuvant treatment is intended to achieve a larger number of conservative surgeries and less complex surgical procedures.

The traditional criteria for initial inoperability are:

- Breast tumor fixed to the rib cage.

- Extensive skin invasion.

- Lymph node cluster fixed to the wall or to an unresectable structure (vascular) (N2).

- Ipsilateral supraclavicular metastases (N3).

- Inflammatory carcinoma.

- Edema of the arm associated with a lymph node cluster.

In some cases, performing an initial conservative procedure will be feasible under very specific selection criteria (e.g., small T4b with N0 or N1). Even if technically resectable, this procedure is not recommended with N2 or N3. 


\section{Criteria for conservative surgical management after primary systemic treatment}

- Prior to neoadjuvant management, primary tumor location and extent marking should be performed.

- After primary systemic treatment, performing imaging studies to assess tumor response is recommended.

- On surgery, tumor residual should be extirpated and the surgical specimen should be marked and oriented for careful histopathological examination. Complete extirpation should be demonstrated with negative margins.

- If tumor disease is found in any margin, it should be broadened to ensure a negative margin; if warranting negative margins with conservative surgery is not possible, total mastectomy should be performed.

- Marking the site of the extirpated tumor (tumor bed) with metal staples is recommended in order for radiotherapy treatment to be more precise. ${ }^{5,6}$

Axillary region ideal management remains controversial. Traditionally, it has consisted of complete axillary dissection or at least levels 1 and 2, either before or after chemotherapy. However, based on current experience, in cases initially regarded as surgical, if the axilla is clinically negative (No) prior to systemic treatment, considering axillary lymph node mapping is feasible, which should be carried out with dual technique (dye and technetium 99 [Tc 99]) in order to obtain an acceptable identification rate.

With current evidence, lymph node mapping after systemic treatment seems a reasonable option without a deleterious impact on local control. 7,8

Breast reconstruction is an option that should be analyzed in all mastectomy-treated cases, even when locally advanced. The surgical and plastic surgery groups shall assess the best technique and timing for reconstruction. Strictly, there is no contraindication for it to be immediate, although the fact that the rate of complications may increase in cases that require post-surgery radiotherapy should be considered. Oncoplastic surgery techniques also enable more complex resections and with better esthetic results. ${ }^{9}$

\section{Radiotherapy aspects}

Generally, radiotherapy in locally advanced disease is postoperatively used and the recommendations mentioned for chest wall and lymph nodes are followed..$^{1-3}$

The indications include:

- T3 or T4 initial tumors.

- Positive surgical margins.
- Four or more positive axillary lymph nodes (N2).

- Post-neoadjuvant chemotherapy conservative surgery.

The recommended dose of radiotherapy to the chest wall and lymph node-bearing zones is 50 Gy. In case of positive margin, administering an additional to the costal wall is recommended.

\section{Inflammatory disease}

Locoregional radiotherapy is recommended as part of the treatment for all patients with inflammatory disease at doses higher than 50 Gy. ${ }^{4,5}$

\section{Radiotherapy-induced toxicity}

At-risk organs tolerance doses should be respected according to the international restrictions established by the Quantitative Analyses of Normal Tissue Effects in the Clinic (QUANTEC) ${ }^{1}$ or in agreement with the RTOG protocols, which limit the dose received by a specific volume of each particular organ. ${ }^{2,3}$

\section{Acute toxicity}

Most patients will develop radioepithelitis from the third week of treatment on. There is no specific therapy to prevent or avoid it.

Ten to $15 \%$ of patients will experience skin fold moist radioepthelitis, which is not a cause for treatment discontinuation. ${ }^{4}$

\section{Sub-acute and chronic toxicity}

\section{Pulmonary toxicity}

Radiographic infiltrate and localized interstitial fibrosis are common when the supraclavicular field is irradiated, with no clinical repercussion. Sub-acute pneumonitis occurs in less than $3 \%$, and it appears as cough that resolves with anti-inflammatory drugs.

When symptoms are severe, treatment consists of short steroid courses. ${ }^{5}$

Irradiated lung volume is a predictive factor for the development of pneumonitis (Table 1).

\section{Cardiotoxicity}

The acute complication is percarditis; at the coronary level, toxicity has a latency period of 10 years or more. Cardiac-origin death in irradiated patients was documented 
Table 1. Dose/volume restrictions according to RTOG*

\begin{tabular}{|l|c|c|c|}
\hline AT-RISK ORGAN & V20 & V10 & V5 \\
\hline Lung & $<20 \%$ & $<35 \%$ & $<50 \%$ \\
\hline $\begin{array}{l}\text { Heart } \\
\text { Left breast }\end{array}$ & $<5 \%$ & $<3 \%$ & \\
\hline $\begin{array}{l}\text { Heart } \\
\text { Right breast }\end{array}$ & $0 \%$ & $<10 \%$ & \\
\hline
\end{tabular}

*These values are interpreted as follows: for example, V $20<20 \%$ indicates that the lung volume that receives 20 Gy should be lower than $20 \%$, V $10<35 \%$ indicates that the lung volume that receives 10 Gy should be lower than $35 \%$.

before 1980 with the use of old 2D techniques. Established volumes for prescription are presented in table $1.6,7$

\section{Second primary tumors}

Absolute risk is relatively low (RR: 1.13); increased patient survival forces for radiation techniques to be improved in order for healthy tissue to be avoided. ${ }^{8,9}$

\section{$\mathrm{XV}$. Metastatic breast cancer treatment}

\section{Introduction}

Metastatic breast cancer is a heterogeneous condition, so far incurable, with variable clinical manifestations and the treatment of which depends on the site and number of metastases, patient characteristics, tumor phenotype and sensitivity or resistance to previous oncologic medical treatments. ${ }^{1}$

This stage of the disease is not curable; however, in coincidence with the introduction of novel and more efficacious systemic treatments, a survival improvement has been observed in the past two decades. ${ }^{2,3}$

The goals of treatment in metastatic breast cancer are:

- To prolong disease-free interval and overall survival.

- To palliate disease-related symptoms.

- To maintain an adequate quality of life with good performance status.

The most important clinicopathological factors to decide the best therapeutic strategy are:1,4

- Age.

- Disease-related symptoms and performance status.

- Concomitant conditions.

- Disease-free interval.

- Number and location of metastases.

- Previous treatment and response to it.

- Hormone receptors and HER-2 neu.

- Patient preferences.
In patients with tumor recurrence, obtaining a biopsy from a metastatic site is recommended to confirm the diagnosis and to determine hormone receptors and HER-2 status, since around $30 \%$ of cases have been shown to change their immunophenotype. This means that an important proportion of patients will have to have their treatment changed in order to avoid insufficient or excessive therapies. Assessment of other biomarkers is not recommended. . $^{1,5-7}$

\section{Treatment according to breast cancer subtype}

\section{A. Metastatic breast cancer With positive HORMONE RECEPTORS AND NEGATIVE HER-2 NEU}

In general, the treatment of choice in this subgroup is hormone therapy, depending on the menstrualstatus. However, in patients with important symptoms and/or rapidly-progressing visceral metastases (visceral crisis*), combination chemotherapy should be the first option, as it produces higher rates of response and palliation.

\section{Hormone therapy in premenopausal patients}

Tamoxifen and/or tamoxifen combined therapy with ovarian suppression/ablation is the first-line treatment of choice in hormone therapy-naive patients. In case of progression with tamoxifen, but with an initial objective response to it, or when patients have recently received previous adjuvant therapy with this drug or exhibit intolerance to it, indicating ovarian ablation or suppression will be possible, ${ }^{1-3}$ and to continue with treatment as recommended for postmenopausal patients.

\section{Hormone treatment in postmenopausal patients}

\section{First line}

The recommended treatment is palbociclib + letrozole. ${ }^{4}$ An aromatase inhibitor is another option in those patients for whom palbociclib is not available. ${ }^{5}$ In those who received an aromatase inhibitor in the adjuvant setting, it is possible using it again in metastatic disease provided the disease-free interval is longer than 1 year.

Another additional first-line possibility is fulvestrant, mainly in patients with no visceral metastases. ${ }^{6}$ 
Table 1. Triple-negative or hormone receptor-positive, HER-2 neu-negative metastatic breast cancer not candidate to hormone therapy ${ }^{8-19}$

\begin{tabular}{|c|c|c|c|c|}
\hline & ADJUVANCY & & & \\
\hline & Not received & Taxane + anthtracycline & Taxane & Anthracycline \\
\hline & Regimen based on: & $\begin{array}{l}\text { - Capecitabine } \\
\text { - Eribulin** } \\
\text { - Gemcitabine } \\
\text { - Vinorelbine }\end{array}$ & Regimen based on: & $\begin{array}{l}\text { Taxane } \pm \\
\text { - Capecitabine } \\
\text { - Gemcitabine }\end{array}$ \\
\hline $1^{\text {st }}$ line & $\begin{array}{l}\text { - Anthracyclin } \\
\text { - Taxane* }\end{array}$ & $\begin{array}{l}\text { - Ixabepilone } \\
\text { - Platinum salts }\end{array}$ & - Anthracyclin & (C) \\
\hline $2^{\text {nd }}$ line & According to previo & treatment & & $\frac{\frac{1}{d}}{\frac{c}{n}}$ \\
\hline $3^{\text {rd }}$ line & According to previo & treatment & & $\frac{7}{7}$ \\
\hline $\begin{array}{l}\text { *In case pa } \\
\text { ** Eribulin } \mathrm{m}^{* *} \text { Only in t } \\
\text { Visceral cr } \\
\text { oresence of } \\
\text { another trea }\end{array}$ & $\begin{array}{l}\text { hosen, it is recommended } \\
\text { monstrated overall surviva } \\
\text { ve tumors. } \\
\text { organ dysfunction as ass } \\
\text { etastases, but implies imp } \\
\text { ot at progression will prob }\end{array}$ & $\begin{array}{l}\text { basis. } \\
\text { patients with triple-negative bre } \\
\text { Ins and symptoms, laboratory stu } \\
\text { al compromise leading to a clini } \\
\text { possible. }\end{array}$ & $\begin{array}{l}\text { apid progression of diseas } \\
\text { tion for a more rapidly effic }\end{array}$ & I crisis is not the mere \\
\hline
\end{tabular}

\section{Second line}

If patients already received a non-steroidal Al (anastrozole/letrozole) or show progression during adjuvant treatment with $\mathrm{Al}$, there are several treatment options: a. Steroidal Als (exemestane). ${ }^{7,8}$

b. Pure anti-estrogen, fulvestrant. ${ }^{9}$

c. Exemestane plus everolimus. ${ }^{10,11}$

d. Fulvestrant + palbociclib, as long as neither drug has been used at first line. ${ }^{12}$

In patients with response or clear initial benefit with hormone therapy and who progress to a first line, second, third and even fourth hormone lines should be tried depending on the used drug, since tumor response is often obtained again, ${ }^{13}$ which means the possibility of chemotherapy-free survival with better quality of life. In case of proven resistance to hormone management, treatment should be switched to chemotherapy. For patients with positive receptors who have received chemotherapy up to maximum benefit, continuing with maintenance hormone therapy is suggested, and the chosen drug shall be administered until progression.

\section{B. Hormone RECEPTOR-POSITIVE, HER-2 NEU- POSITIVE METASTATIC BREAST CANCER}

The recommended treatment in this group of patients is chemotherapy plus anti-HER-2 therapy (see HER-2-positive, hormone receptor-negative breast cancer section). ${ }^{1,2}$

In postmenopausal patients in whom chemotherapy is not indicated, an aromatase inhibitor plus lapatinib or trastuzumab is recommended. . $^{3,4}$

In patients receiving treatment with chemotherapy plus anti-HER-2 therapy, continuing treatment with
anti-HER-2 therapy plus single-agent hormone therapy is considered adequate after chemotherapy completion or discontinuation.

\section{TRIPLE-NEGATIVE OR HORMONE RECEPTOR- POSITIVE, HER-2 NEU-NEGATIVE METASTATIC BREAST CANCER NOT CANDIDATE TO HORMONE THERAPY}

Treatment selection should take into account whether adjuvant treatment was previously administered (table 1) and the interval between its conclusion and recurrence. In patients with an interval longer than 1 year, pharmacological re-induction can be evaluated, with tolerance and cumulative dose being considered in all cases. For patients with triple-negative tumors, the only treatment option is chemotherapy, without recommending a specific regimen or sequence being currently possible..$^{1,2,5}$

\section{First-line chemotherapy: in combination or sequential?}

Polychemotherapy is not recommended as standard treatment. Single-drug and sequential treatment is preferred due to better tolerance and quality of life. The use of polychemotherapy should be evaluated, if patient conditions allow, only in those in whom rapid response or symptom and/or visceral crisis* palliation is sought and/or in cases where life expectancy is considered to allow only one treatment opportunity. 1,2,6,7

The cornerstone of first-line chemotherapy is based on anthracyclines and taxanes. In patients with previous exposure, treatment options include capecitabine, gemcitabine, vinorelbine, ixabepilone or eribulin mesylate (Table 1). 
In case a combination is chosen, a taxane plus capecitabine or gemcitabine is recommended. Both regimens have been associated with higher response rates and longer progression-free interval versus taxane monotherapy. The efficacy of both regimens is similar and the choice will depend on each patient's characteristics and available resources.

The choice of treatment depends on patient characteristics, tolerance and response to previous treatments, as well as availability. The points of interest to be taken into account include the following:

The best results with paclitaxel are obtained when administered on a weekly basis.

In patients with previous adjuvant treatment and disease-free interval shorter than 1 year, re-induction with previously used drugs is not recommended.

Eribulin mesylate is the only drug that has shown an impact on overall survival in patients previously treated with taxanes/anthracyclines, especially in population with triple-negative tumors.

Ixabepilone is recommended as single-drug, except in cases where rapid palliation is desired, where its use combined with capecitabine can be evaluated according to patient characteristics.

\section{Platinum salts}

There are studies demonstrating the effectiveness of platinum and its derivatives in triple-negative tumors. ${ }^{20,21}$ The TNT phase III trial assessed the use of docetaxel versus carboplatin and demonstrated the platinum salt non-superiority in non-selected triple-negative population (mutated versus non-mutated BRCA); however, in the population with BRCA mutation present, superiority in progression-free survival was observed in favor of carboplatin. ${ }^{22}$ Although platinum salts are not recommended as first-line therapy in non-selected population, they can represent an option in population with the BRCA mutation.

\section{BeVACIZUMaB}

The use of bevacizumab plus a chemotherapeutic agent increases disease control and progression-free survival, but does not impact on overall survival as first-line therapy in metastatic breast cancer. ${ }^{23-27}$ For the purposes of this Consensus, bevacizumab plus taxane is considered to be a treatment option in patients with triple-negative tumors or in those with positive hormone receptors and a clinically aggressive evolution who are considered candidates to first-line chemotherapy.

\section{TREATMENT DURATION}

Treatment duration has not been entirely defined. Several studies have demonstrated that continuing chemotherapy may increase the progression-free interval, but without survival being prolonged. ${ }^{28,29}$

In clinical practice, continuing chemotherapy is recommended until progression or toxicity, depending on the applied drug (intravenous versus oral), cumulative maximum doses and patient quality of life.

\section{HER-2 NEU-POSITIVE METASTATIC BREAST CANCER}

First-line standard treatment for this group of patients is docetaxel plus trastuzumab and pertuzumab, since it has clearly demonstrated a benefit in overall survival, progression-free survival and response rates. ${ }^{30,31}$

In patients who cannot receive pertuzumab, trastuzumab plus taxane should be considered as an alternative. ${ }^{32}$ In selected cases, vinorelbine plus trastuzumab can be an option when taxane is not indicated. ${ }^{33}$

In patients previously treated with a trastuzumab and pertuzumab-based regimen and with disease progression, second-line-indicated treatment is TDM- $1 .{ }^{34}$

Third and subsequent lines regimens are based on the administration of lapatinib plus capecitabine, lapatinib plus trastuzumab or trastuzumab plus a chemotherapeutic agent. ${ }^{35-37}$

Maintaining the blockade with anti-HER-2 therapy is recommended in all patients during all phases of antineoplstic treatment, except in cases where it is contraindicated, since its impact on disease control is demonstrated. ${ }^{1,2,36,37}$

\section{The role of surgery in metastatic disease}

Survival prognosis for patients with stage IV breast cancer has improved over the past few years. With multi-modal treatment, 5-year survival of $23.4 \%$ has been reported. The role of surgery for patients in this situation is controversial, and some authors consider it an option for survival to be increased., ${ }^{1,2}$

In patients with metastatic breast cancer, surgical treatment can be evaluated in three scenarios:

a. Metastatic disease resection (primordially hepatic and/or pulmonary).

b. Primary tumor resection in the presence of distant metastasis.

c. Palliative tumor resection in the presence of ulceration or bleeding. 


\section{A. Metastatic disease resection}

\section{LIVER METASTASES}

Some patients diagnosed with metastatic breast cancer present it at the hepatic level, and in one third of them this is the only site of distant disease. Many studies have evaluated hepatic resection in patients with metastatic breast cancer. The 5-year survival rate after liver metastases surgical resection has been reported to range from $18 \%$ to $61 \% .{ }^{2}$ Current surgical techniques allow for resection to have postoperative mortality lower than $6 \%$ and morbidity ranging from $0.8 \%$ to $5.4 \%$ in reference centers. ${ }^{2}$ Another valid option is to use metastasis ablation with radiofrequency or laser-induced interstitial thermotherapy, which report mean survival from 30 to 60 months and 5 -year survival from $27 \%$ to $42 \% .^{3}$

With regard to prognostic factors, most studies emphasize on the importance of R0 resection, since positive margin is an adverse prognostic factor in many cases. ${ }^{3,4}$ Other adverse predictors for survival have been hormone receptor status, poor response to chemotherapy, vascular invasion, number of metastases and disease-free interval $<1$ year after breast cancer primary resection. Based on the above, patients with positive receptors, disease-free interval $>1$ year with good response to preoperative chemotherapy and single metastasis or oligometastases where $\mathrm{R} 0$ resection is possible should be considered for breast cancer liver metastases resection or ablation. ${ }^{2-4}$

\section{Pulmonary metastases}

Lung metastases complete surgical resection can be performed with low morbidity and mortality. Several retrospective studies have observed that $15 \%$ to $25 \%$ of patients with breast cancer metastases present them on the lung or pleural space. Five-year survival reaches between $27 \%$ and $54 \% .5-6$

A common finding in most studies assessing the role of lung metastases resection is that the disease-free interval between the primary tumor and the appearance of lung metastases has a highly significant impact on survival. Other factors that have been associated with survival improvement are positive ER, positivity for HER-2 neu and solitary metastases. ${ }^{5,6}$ As in the case of liver metastases, patients with single metastasis and prolonged disease-free interval should be considered candidates to pulmonary metastasectomy.

\section{OtheR METASTATIC SITES}

This type of groups is the least studied and has not shown survival benefit. An example is brain metastases, since these patients have an unfavorable prognosis, although the usefulness of palliative resection has been suggested. ${ }^{7}$ Another example is bone metastases; according to several reports, surgical resection has not shown prognostic benefit in these patients. ${ }^{8}$ In both cases, radiotherapy is the palliative modality of choice. On the other hand, some studies have found that sternum or rib cage metastasis resection is associated with survival increase. ${ }^{9}$ Less studied owing the their infrequency are adrenal, ovarian and gastrointestinal metastases; in these cases, resection is not recommended except for situations of symptom palliation.

\section{B. Primary tumor resection in metastatic disease}

This is a clinical scenario where controversies are even bigger and evidence is also scarce, since potential recommendations are based on retrospective studies with significant selection bias. Several studies, both institution and population-based, have demonstrated a survival advantage when primary tumor resection is performed in patients with stage IV breast cancer. ${ }^{10,11}$ Leaving the tumor in situ has been proposed to be a potential source of new metastases, and its removal would therefore reduce the possibility of disease progression. On the other hand, tumor volume reduction might increase chemotherapy efficacy by decreasing the likelihood of resistant cellular clones' occurrence. ${ }^{10}$

All studies assessing this problem are retrospective and patients were not randomly selected for surgery but were assigned by the treating physician, generally based on lower tumor burden, absence of visceral metastases and younger age, among other factors. Studies that have statistically controlled for these variables, have found no benefit from tumor removal, and we must therefore wait for the results of ongoing randomized prospective trials, which are essential to know if locoregional treatment can improve the prognosis in patients with metastatic breast cancer. ${ }^{12-14}$

Therefore, primary tumor resection in the presence of metastases would currently be recommended for palliative purposes (primary lesion ulceration or imminent ulceration) in order to improve quality of life, without an 
impact on survival. Available data indicate that it is reasonable to select patients with favorable clinical characteristics, specifically young age, good general condition, positive hormone receptor disease, only bone disease and limited tumor volume, or else patients who have received upfront systemic treatment with an excellent response, to undergo locoregional treatment. ${ }^{10-16}$

\section{Primary tumor palliative resection in metastatic disease}

In this clinical scenario there is no controversy: surgery is indicated when there is tumor ulceration or hemorrhage, if it is resectable with low morbidity. In case of non-resectable primary tumors, palliative radiotherapy may be considered.

\section{THE ROLE OF RADIOTHERAPY IN METASTATIC DISEASE}

The benefit of radiotherapy to the primary site in patients with metastatic breast cancer is controversial; retrospective and prospective trials show positive results in local control, whereas reports on overall survival benefit are not consistent, and its indication should therefore be individualized within the context of a multidisciplinary analysis. ${ }^{1-3}$

Metastatic disease treatment distinguishes two groups of patients according to different characteristics: a group including patients in good general conditions, controlled primary group and disease confined to three sites, and another group with poor performance status or extensive metastatic dissemination.

In patients with controlled primary tumor and oligometastatic disease, the use of ablative resources such as radiosurgery or stereotactic body radiotherapy (SBRT) is warranted, whereas patients with clinical deterioration or multiple metastatic dissemination require the relief of symptoms such as pain, bleeding or skin involvement with short schemes of palliative radiotherapy.

\section{Bone METASTASES}

Palliative radiotherapy schemes include 37.5 Gy in 15 sessions, 30 Gy in 10 sessions, 20 Gy in 5 sessions or a single dose of $8 \mathrm{~Gy}$. The effectiveness in pain control between schemes has been shown to be equivalent; however, the shorter the radiotherapy course is, the higher the retreatment rates will be, and life expectancy should therefore be considered for better treatment selection. ${ }^{5}$

\section{BRAIN METASTASES}

Surgery is reserved for bulky, single and symptomatic lesions. Radiotherapy modalities include: whole brain radiation, stereotactic radiosurgery (SRS) or both. Whole brain radiotherapy is used in multiple metastatic brain lesions, non-controlled primary tumor or poor performance status. Stereotactic radiosurgery is generally recommended for $\leq 3$ lesions and $<3 \mathrm{~cm} .^{6}$

Radiosurgery improves survival in patients younger than 50 years. Whole brain radiotherapy addition after radiosurgery decreases the risk of new brain lesions appearance, with no impact on survival and with a deleterious effect on patient memory. ${ }^{7}$

Meningeal carcinomatosis is a separate entity and is treated for palliative purposes.

\section{SBRT in oligometastatic disease}

Oligometastatic disease means limited metastatic dissemination that is potentially curable with local treatment and that generally is described as $\leq 5$ metastatic sites.

\section{A. SBRT in liver metastases}

Stereotactic radiotherapy is indicated in patients with liver metastases who are not candidates to surgical management or refuse surgery. The conditions for this technique are: women with adequate performance status ECOG 0-1, absent or stable extra-hepatic disease, $\leq 3$ lesions, $<3 \mathrm{~cm}$, good liver function and liver volume $>1000 \mathrm{~cm}^{3}$.

The borderline group includes: patients with 4 lesions, 4 to $6 \mathrm{~cm}$ in diameter, moderate liver function and functional liver volume of 700 to $1000 \mathrm{~cm}^{3.8,9}$

\section{B. SBRT in lung metastases}

SBRT has been shown to be superior in local control and survival in comparison with external beam radiotherapy and to produce equivalent control rates to surgical treatment. SBRT in lung metastases provides local control at 1,3 and 5 years of $80 \%, 58 \%$ and $46 \%$, respectively. It is associated with higher survival in small lesions of < $11 \mathrm{cc}$ in volume; one biological equivalent dose $(B E D) \geq 100$ Gy results in better local control. ${ }^{10}$ Complications are low and can include asthenia, adynamia, cough, pneumonitis and costal fracture. 


\section{Spinal SBRT}

Indications include: KPS $>60$, demonstrated metastatic disease, single or multiple lesions ( $\leq 2$ consecutive vertebrae or up to 3 non-contiguous sites), no data consistent with spinal cord compression or pathologic fracture, residual or recurrent disease after surgery and with an interval longer than 6 months in cases of re-irradiation. ${ }^{11}$

\section{Other metastatic lesions}

Palliative radiotherapy is also used in case of invasion to the skin, soft tissue and other less common sites such as ocular lesions.

\section{Bisphosphonates and receptor activator of NF-KB ligand (RANKL) inhibitors in bone metastases, adjuvancy and with aromatase inhibitors}

Both bisphosphonates and receptor activator of NFKB ligand (RANKL) inhibitors allow improving the results in the management of bone metastases, ${ }^{1}$ malignant hypercalcemia and bone health by reducing osteopenia or osteoporosis secondary to systemic treatment. ${ }^{2,3}$

\section{Bone metastases}

- Patients with radiographic evidence of bone metastases should receive treatment either with denosumab (120 mg subcutaneous every 4 weeks), ${ }^{4}$ zoledronic acid (4 $\mathrm{mg}$ by intravenous [IV] route in 15 minutes $)^{5,6}$ or pamidronate (90 mg IV in 2 hours) every 3 to 4 weeks. ${ }^{5,7}$

- Total duration of treatment with bisphosphonates should be up to 2 years. Zoledronic acid can be applied every 3 to 4 weeks or every 3 months from the beginning. ${ }^{8}$ After 1 year of treatment, and in case of stable disease, zoledronic acid administration is recommended every 12 weeks during the second year ${ }^{9}$ and then reconsidering its use according to bone metastases activity. Optimal duration of denosumab treatment is not known.

\section{Adjuvant therapy}

Bisphosphonates and denosumab administration as adjuvant therapy is not recommended. ${ }^{10,11}$

\section{Aromatase inhibitors (AI)-related bone loss}

Patients starting with an Al should undergo hip and column bone mineral density measurement, ${ }^{11-14}$ as well as an assessment of risk factors for fracture following the behaviors indicated in figure 1 .

Zoledronic acid at $4 \mathrm{mg}$ IV is recommended every 6 months for the 5 years of Al therapy or denosumab $60 \mathrm{mg} \mathrm{SC}$ every 6 months for 2 years.

Bone remodeling biomarker determination is not routinely used in patients receiving $\mathrm{Al}^{13,14}$

\section{Recommendations with the use of bisphosphonates and RANKL inhibitors}

- Dental examination prior to their administration.

- Oral cavity examination every 6 to 12 months.

- Avoid dental surgeries during treatment.

- Not recommended in patients with preexisting oral infections or poor oral hygiene.

- Zoledronic acid is contraindicated in patients with creatinine clearance $<30 \mathrm{ml} / \mathrm{min}$.

- Denosumab should be used with caution in patients with creatinine clearance $<40 \mathrm{ml} / \mathrm{min}$.

- The patient should receive calcium $(1200 \mathrm{mg})$ and vitamin D (1000 mg) supplementation everyday. ${ }^{15}$

- Control bone mineral density measurement every 1 to 2 years.

\section{$\mathrm{XVI}$. Breast cancer in young women}

This Consensus considers an age cutoff of 40 years to define young patients. This benchmark is based on differences observed with regard to risk factors, tumor characteristics and clinical outcomes, as well as on particular interests: fertility, self-image, quality of life perception and personal goals, when compared with women exceeding this age limit.

The following are concepts associated with diagnosis and recommended treatment for this group of patients:

- Young age alone should not be a reason to prescribe more aggressive therapy than generally recommended. ${ }^{1,2}$

- Multidisciplinary treatment is highly recommendable, as well as individual treatment planning in the following aspects:

- Personalized psychosocial support.

- Genetic counseling. 


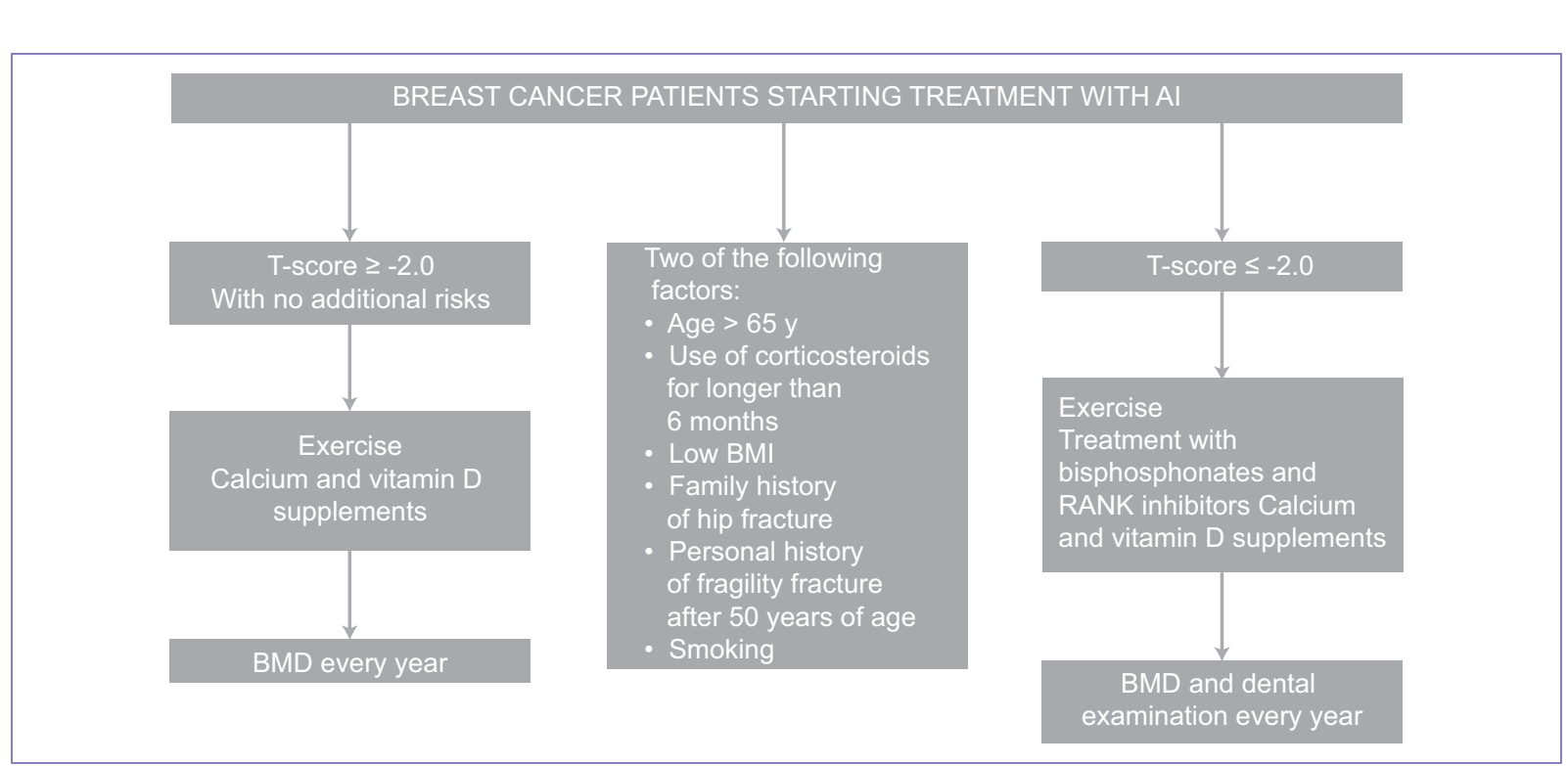

Figure 1. Flow-chart to assess fracture risks.

- Reference for ovarian reserve and fertility preservation.

- Approach to sexual and body image disturbances.

- Diagnosis, imaging studies and staging in young women should follow standard algorithms consistent with those for older women (see relevant section). Further consideration can be given to breast US and MRI in young women, particularly in patients with extremely dense breast tissue or genetic predisposition.

- The recommendations for surgical treatment of young women with early breast cancer -although treatment should be individualized-should not differ from those indicated for older patients.

- Although young age is an independent risk factor for local recurrence, ${ }^{3}$ treatment with breast-preserving surgery and radiotherapy does not affect overall survival when compared with surgical treatment with mastectomy and should be considered an option for this group of patients. ${ }^{4-6}$

- In case of treatment with conservative surgery, after finishing adjuvant radiotherapy, offering a 16-Gy boost to the tumor bed to all young patients is recommended, given that the 5-year risk for locoregional recurrence is significantly decreased (from 20 to 10\%).,

- Patients with hormone-sensitive breast cancer should receive endocrine therapy for at least 5 years (see relevant section). If a $\mathrm{GnRH}$ analogue is used in this age group, it should be administered monthly (not every 3 months) in order to optimize ovarian suppression efficacy. ${ }^{9}$ Ovarian suppression adequacy should be verified by measuring estradiol levels (months 0,3 , $6,12,18,24,36,48$ and 60$).{ }^{10}$ In case of inadequate suppression, alternative strategies should be discussed (oophorectomy or continuation with tamoxifen alone).

- Indications for adjuvant chemotherapy and radiotherapy are the same as for other patients. The recommendations for advanced breast cancer management also don't differ from those for the other age group (see relevant sections).

- Every young woman aged 40 years or less and with breast cancer should be offered genetic counseling, regardless of the breast cancer subtype (see relevant section).

- Women who did not receive counseling at the moment of breast cancer diagnosis, should be offered it during follow-up in order to address monitoring issues and strategies to reduce the risk for additional primary tumors in the patient and her family.

- All young women should be informed and advised about related amenorrhea and premature menopause risks and symptoms resulting from systemic treatment prior to its initiation (chemotherapy or endocrine therapy).

- Young women should be advised to seek fertility and contraception specialized counseling prior to making any treatment decision.

- Monthly administration of GnRH analogues concomitantly with chemotherapy can be considered in premenopausal women with breast cancer who are interested on preserving ovarian function and/or fertility. ${ }^{11}$ Their use in patients with positive and negative hormone receptors does not confer risk for recurrence. ${ }^{12,13}$ 
- The use of exogenous hormone contraceptives is generally contraindicated in young survivor women and alternative strategies should be considered:

- If the patient has completed her childbearing plans: look for definitive options (bilateral tubal occlusion or vasectomy).

- If the patient has not completed her childbearing plans: IUD (copper-T). The use of levonorgestrel-releasing IUD is controversial.

- Another option for patients with non-completed childbearing plans: condom (consider failures associated with incorrect use).

- Inquire on hormone contraceptives use and recommend discontinuation.

- Patients should be informed on the possibility of pregnancy even during endocrine therapy in spite of the presence of amenorrhea and on the need for an adequate non-hormone contraceptive.

- Performing a pregnancy test is recommended prior to the start of systemic treatment with chemotherapy and/or hormone therapy.

- Detriment on the prognosis of patients with pregnancies subsequent to breast cancer diagnosis has not been demonstrated. ${ }^{14,15}$ Doctors should discuss this possibility case by case with those interested in attempting to get pregnant and not discourage their maternity desire..$^{1,16}$

- The timing for pregnancy attempt should be personalized taking into account patient age and ovarian reserve, previous antineoplastic treatments and time from completion, as well as individual relapse risk. ${ }^{16}$ In general, trying to get pregnant is recommended 2-3 years after chemotherapy conclusion in patients with hormone-negative tumors. ${ }^{17}$ For patients with hormone-sensitive breast cancer, the POSITIVE trial is active, which allows anti-hormone treatment temporary discontinuation for 2 years. ${ }^{18}$

- Treatment-related premature menopause and/or amenorrhea increase the risk of bone density decrease in premenopausal women, and monitoring and accordingly treating it is therefore recommended (see relevant section).

\section{$\mathrm{XVII}$. Treatment in advanced age patients}

This Consensus considers a cutoff age of more than 70 years to define "woman with advanced age". This group of patients corresponds to a heterogeneous group of patients where physiological age is not necessarily a reflection of biological age. There is a general division of the elderly population ${ }^{1}$ where a direct relationship between age and life expectancy is established: a) young elderly women 65 to 75 years with life expectancy of 15 years, b) old elderly women: 76 85 years with life expectancy of 10 years and c) old-old elderly; women $>85$ years with life expectancy of 5 years.

According to Balducci, ${ }^{2}$ this population is classified as follows:

- Healthy: functionally independent, without major comorbidities; they are candidates to receive onco-specific treatment in standard conditions.

- Vulnerable: these are partially independent patients with no more than 2 comorbidities; they benefit from curative intent modified treatment.

- Frail: dependent patients with 3 or more comorbidities or presence of geriatric syndrome, and are therefore candidates to symptomatic and/or palliative treatment.

Current scientific evidence ${ }^{3-5}$ does not allow for specific treatment recommendations to be established for this population because this patient group is rarely included in most clinical trials. However, taking into account the following factors is suggested:6-10

Age by itself should not dictate the treatment decision, ${ }^{5}$ performing a geriatric evaluation is suggested in order to define if the patient is healthy, frail or vulnerable, which will enable to provide a treatment where comorbidities will be assessed ${ }^{4,5}$ in order to obtain better therapeutic indices. Explaining the treatment goals to the patient and her family is necessary; those with life expectancy $>10$ years should receive the same management as younger patients. $^{5}$

Performance status (Cancer and Aging Research Group), geriatric evaluation and chemotherapy-related toxicity predictor are listed in table 1 (www.mycarg. org). ${ }^{11,12}$

\section{Surgery in women with advanced age}

According to the recent literature, the recommendation in this patient group is that age is not a factor that determines the choice of surgical treatment; risk should be assessed based on comorbidities ${ }^{6}$ and, in some cases, the alternative of medical treatment is considered.

\section{Systemic treatment}

Specific recommendations for geriatric patients based on their general condition are shown in table 2. 
Table 1. Cancer and Aging Research Group (CARG) predictors of chemotherapy-related toxicity in patients with advanced age $^{12}$

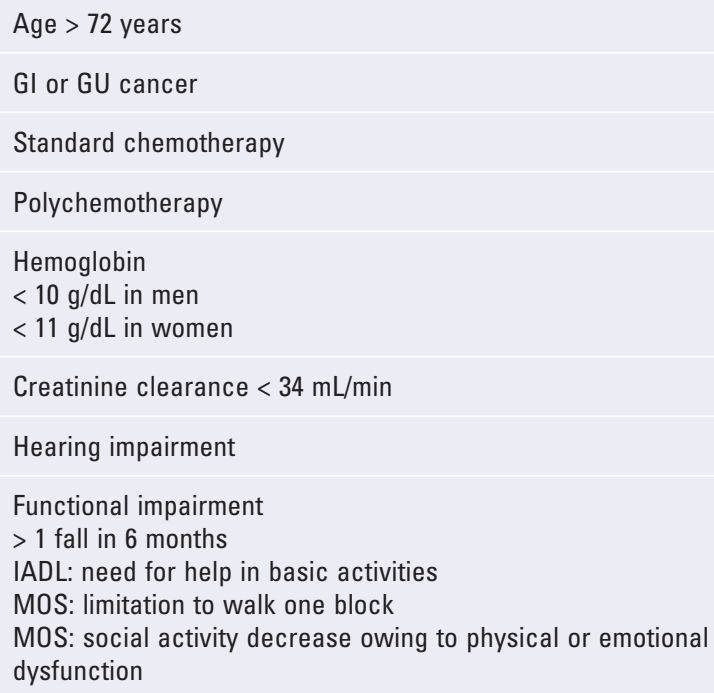

IADL: Instrumental activities of daily living; GI: gastrointestinal; GU: genitourinary MOS: Medical Outcomes Study.

Definitive or primary endocrine treatment is recommended in non-operable patients, with positive hormone receptors and life expectancy of less than 2 years.

In patients with low-grade $\mathrm{T}<1 \mathrm{~cm}$, N0, or patients with serious comorbidities, adjuvant hormone therapy can be omitted.

Standard chemotherapy treatment should be considered in every patient with life expectancy higher than 10 years (ePrognosis.ucsf.edu; 10-year mortality risk $<5 \%$ ).

\section{Radiotherapy in women with advanced age}

According to the Society of Geriatric Oncology (SIOG)/European Society of Breast Cancer (EUSOMA) 2012 guidelines, ${ }^{3,6}$ breast cancer treatment in this age group should be based on geriatric evaluation. Radiotherapy treatment is proposed in table 2 .

\section{Radiotherapy recommendations}

In women managed with conservative surgery, ${ }^{10}$ adjuvant radiotherapy treatment administration impacts on locoregional control without an overall survival or disease-free survival benefit, and it is therefore indicated in patients with the following criteria: ${ }^{11-13}$
- Women aged $\geq 70$ years, clinically negative lymph nodes, ER+, T1 (category 1), negative margin and willing to take endocrine treatment.

- Willingness to accept $10 \%$ of local recurrence at 10 years.

For patients undergoing mastectomy, the same criteria will be employed to indicate radiotherapy; clinical condition and comorbidities should be considered to evaluate hypofractionation schemes in some cases. In frail patients who are not candidates to surgery owing to their general condition or comorbidities, administering only radiotherapy may be evaluated. ${ }^{14}$

\section{Male breast cancer}

Male breast cancer accounts for less than $1 \%$ of total breast cancer cases. ${ }^{1}$ Main risk factors are BRCA 2 gene mutation, Klinefelter syndrome, cryptorchidism, previous radiotherapy to the chest and use of exogenous estrogens. ${ }^{2,3}$

The predominant histological type is ductal invasive, present in about $90 \%$ of cases. The vast majority have positive hormone receptors (90\% to $95 \%$ ), whereas HER-2 neu is positive only in $11 \%$ of tumors.

Breast cancer treatment in man has been practically "extrapolated" from data available on breast cancer in women, and it is treated similarly stage by stage, taking patient age and general health condition into account, as well as tumor pathological characteristics, including hormone receptor and HER-2 neu expression.

Recommended local treatment is modified radical mastectomy with sentinel lymph node or axillary dissection according to clinical stage. Breast-preserving surgery is not indicated. Recommendations for radiotherapy include: large tumor size, extension to the skin, areola or pectoralis major muscle, lymph node involvement, retroareolar location and surgical margins compromise. ${ }^{4}$

Adjuvant systemic management follows the same guidelines than in women. Tamoxifen for 5 years is recommended as standard treatment in patients with positive hormone receptors. The use of tamoxifen for 10 tears follows the same guidelines than in women as well. Aromatase inhibitors are not indicated. The use of genomic platforms such as Oncotype, Mammaprint or Endopredict for the decision on adjuvant treatment is not recommended because there is not enough information to evaluate their usefulness. On the other hand, even when there is no evidence on adjuvant trastuzumab benefit in men with HER-2 neu-positive breast 
Table 2. Management algorithm in elderly patients with breast cancer ${ }^{2}$

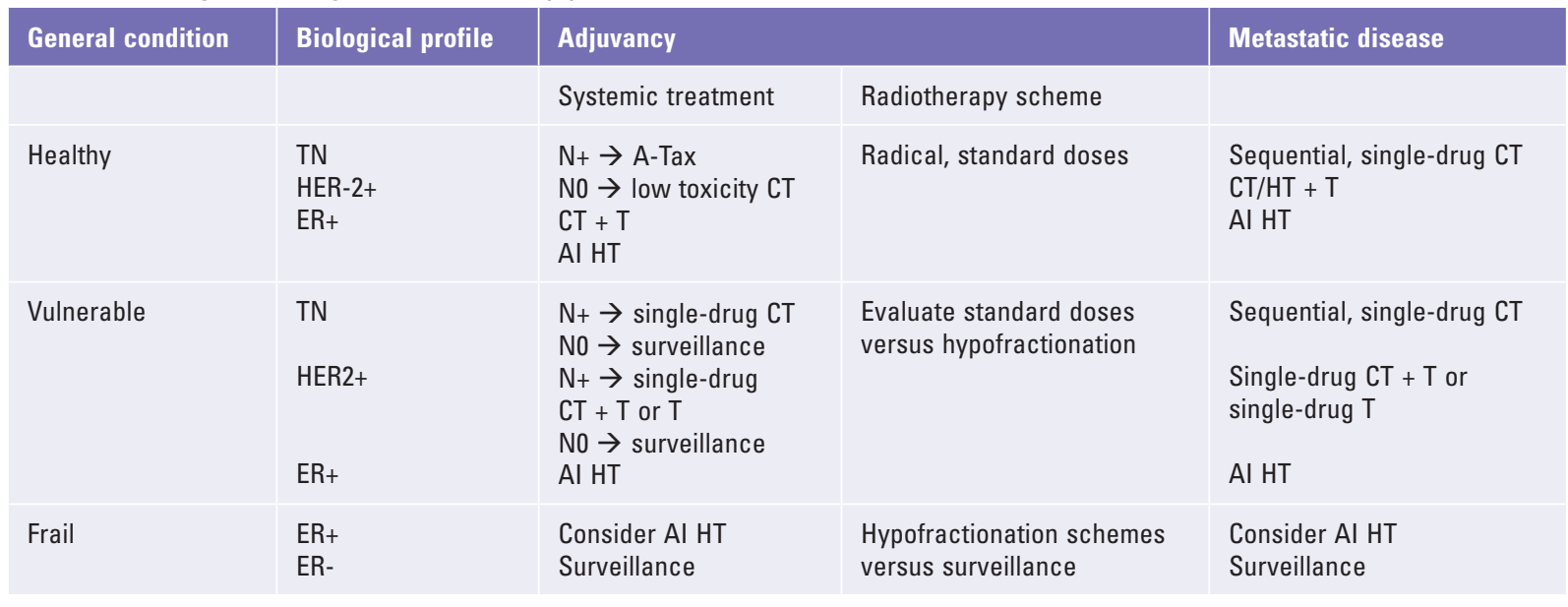

A: anthracycline; Al: aromatase inhibitor; CT: chemotherapy; ER: estrogen receptor; HT: hormone therapy; N: lymph nodes; T: trastuzumab; Tax: taxane; TN: triple-negative.

cancer, its use should be considered according to established indications. ${ }^{4}$

As for locally advanced breast cancer, many patients are diagnosed at that stage and should be treated following the guidelines proposed for women.

In metastatic disease with positive hormone receptors, tamoxifen is regarded as the treatment of choice, except in cases of rapidly growing tumors or with visceral metastases, where looking for a prompt objective response with cytotoxic therapy is necessary. Finally, in patients with negative receptors or hormone-refractory, chemotherapy with equal regimens and doses to those used in women is the treatment of choice. Patients with HER-2 neu-positive tumors should be assessed for the addition of trastuzumab and pertuzumab to their systemic treatment based on the same guidelines than for women. ${ }^{5}$

\section{Breast cancer associated with pregnancy and breastfeeding}

\section{General guidelines}

- Cancer associated with pregnancy is defined as cancer diagnosed during the period of gestation, breastfeeding or within the first year after delivery. ${ }^{1}$

- Physiological changes of the mammary gland during gestation and breastfeeding hinder and delay diagnosis. $^{1,2}$

- The preferred initial imaging study is breast ultrasound. ${ }^{1,2}$

- Mammography should be performed with abdominal protection and be requested if there is suspicion of multicentrality $\mathrm{o}$ bilaterality.
- Core needle biopsy is preferred to corroborate the diagnosis; it is important to inform about the pregnant state of the patient to the pathology department that will handle the specimens. ${ }^{2,3}$

- Suggested disease extent workup includes: ${ }^{4,5}$ - Chest X-ray with abdominal protection.

- Liver ultrasound.

- Thoracolumbar vertebral column magnetic resonance imaging without contrast material in case of suspected bone disease.

- Computerized tomography and nuclear medicine studies should be avoided. 4,5

- Treatment of the pregnant woman should be multidisciplinary and include the oncology and obstetric teams. ${ }^{5}$

\section{Surgery}

- Surgery is a safe procedure at any trimester of pregnancy. 4,6 However, breast-preserving surgery is indicated in the second and third trimester of gestation followed by radiotherapy at the end of pregnancy. ${ }^{6}$

- Axillary standard treatment is level I and II dissection. Scientific information is limited with regard to the performance of the sentinel lymph node procedure during pregnancy. However, some centers have had experience with the use of vital stains such as methylene and patent blue. The radiocoloid technetium 99 can be used at third trimester of gestation; fetus exposure to radiation is estimated to be $4.3 \mathrm{mGy}$. ${ }^{4,6,7}$

\section{Radiotherapy}

Treatment with radiotherapy is contraindicated during the entire pregnancy owing to its teratogenicity and 
malignant neoplasm induction, as well as hematological alterations. ${ }^{8}$

\section{Systemic treatment}

\section{Chemotherapy}

- Chemotherapy is recommended from the second trimester of gestation on. $4,9,10$

- Anthracycline-based regimens are the recommended ones. There is limited experience with taxane administration during pregnancy; they are indicated when there is progression or contraindication to the use of anthracyclines. ${ }^{6,10,11}$

- Chemotherapy administration should be avoided after gestation week 35 in order to prevent obstetric complications. ${ }^{12}$

- In utero exposure to chemotherapy after the second trimester does not affect cognitive, cardiac and physical development of the baby. ${ }^{13}$

Biologic therapies

- The use of adjuvant trastuzumab is contraindicated. The use of any other biologic therapy during pregnancy is not recommended. ${ }^{14}$

- Endocrine therapy

- Tamoxifen administration is contraindicated during pregnancy. ${ }^{4}$

Anti-emetics and supportive therapies

- The use of bisphosphonates is not recommended. ${ }^{4}$

- Antiemetic drugs and colony-stimulating factors should be used according to usual management recommendations. ${ }^{4,15}$

Delivery and breastfeeding

- Breastfeeding should be avoided if the patient is receiving systemic therapy or radiotherapy. ${ }^{10}$

\section{Prognosis}

- Early termination of pregnancy does not improve survival. ${ }^{16}$

- There is contradicting information to consider the presence of pregnancy by itself as an independent poor prognosis factor. ${ }^{16,17}$

\section{Follow-up after curative intent treatment}

At the conclusion of breast cancer primary treatment, usually with surgery, chemotherapy and radiotherapy, begins the surveillance and control stage known as "follow-up".
Internationally-accepted recommendations for the follow-up of these patients are described in table 1. It is important highlighting that the appearance of metastasis after adequate primary treatment is unrelated to medical intervention; in addition, anticipating the diagnosis of relapse does not increase survival or quality of life.

\section{Hormone replacement therapy (HRT)}

HRT administration has been shown to increase the risk for the development of breast cancer (HR: 1.66) and for death from this disease (HR: 1.22), which is related to the fact that it is estrogen-based. ${ }^{1}$ The risk is applicable to the various presentations (oral, vaginal, transdermal); 2,3 in addition, the risk is acknowledged to be higher in current users with more than 5 years of combined hormone therapy (estrogens and progesterone; HR: 2). ${ }^{4,5}$

There are only few studies on the use of HRT in women with previous history of breast cancer, and most are retrospective or prospective non-controlled. ${ }^{2-8}$ On the other hand, there are only two randomized trials, but with conflicting results, which precludes establishing evidence-based conclusions. So far, only one of them demonstrates higher risk: the HABITS trial, which is a double-blind study in cancer survivors with HRT that was stopped in 2003 owing to an increase in cancer risk. ${ }^{9}$ An update of this study demonstrated a 5-year cumulative increase in the incidence of new cancer events in survivors with the use of HRT $(22.2 \%$ in the HRT arm vs. $8 \%$ in the control group), ${ }^{10}$ which was statistically significant.

Although tibolone has been used as an alternative for the management of menopausal symptoms, its administration is not recommended owing to an increased risk for both locoregional and systemic recurrence (HR: 1.4) in women with previous history of breast cancer according to the results of the LIBERATE trial. 11

Based on the above, international guidelines and this Consensus contraindicate the use of HRT in women survivors of breast cancer.

\section{Genetics and breast cancer}

Out of all patients diagnosed with breast cancer, $5 \%$ to $10 \%$ are carriers of a hereditary syndrome, and $25 \%$ to $40 \%$ of them are younger than 35 years. Approximately $20 \%$ of patients have first or second-degree relatives with a history of breast cancer, which is considered a familial presentation. In this case, age at diagnosis is 
Table 1. Recommendations for follow-up

\begin{tabular}{|c|c|c|}
\hline Procedure & Frequency & \\
\hline Instruction to the patient about signs and symptoms of recurrence & At the conclusion of radical treatment & $\stackrel{\infty}{-}$ \\
\hline Physical examination & \multicolumn{2}{|c|}{$\begin{array}{l}\text { First } 2 \text { years every } 3 \text { to } 4 \text { months. Third to fifth year eve } \\
6 \text { months. From the fifth year on, annually }\end{array}$} \\
\hline Breast self-exam & Monthly & 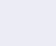 \\
\hline Mammography & Annually & \multirow{2}{*}{$\frac{2}{2}$} \\
\hline Tumor markers & Not recommended & \\
\hline Chest, abdomen CT, PET, bone scan and liver enzymes & Only if there are specific symptoms & $\check{\omega}$ \\
\hline Screening for other tumors (cervical, colorectal, ovarian, endometrial, etc.) & Follow early detection guidelines & $\underline{\underline{n}}$ \\
\hline Instructions to the patient on exercise, physical activity and weight control & On every consultation & ○ \\
\hline
\end{tabular}

consistent with what is expected for the general population and risk factors that contribute to the development of this disease, which is different from hereditary cancer syndromes, can even be identified. ${ }^{1,2}$

Genes related to hereditary breast cancer can be divided into those that confer high susceptibility for the development of cancer (higher than 50\%) (BRCA1, BRCA2, CDH1, NF1, PTEN, TP53 and STK11), moderate susceptibility (20-50\%) (ATM, BRIP1, CHEK2, $P A L B 2, R A D 50$ and NBS1) and low susceptibility (less than 20\%) (FGFR2, LSP1, MAP3K1, TGFB1 and TOX3). ${ }^{3}$

The prevalence of germline mutations in the $B R C A 1$ and $B R C A 2$ genes in the general population has been calculated to be $0.1 \%$ to $0.2 \%$, with mutations in these genes being responsible for $3 \%$ to $8 \%$ of all cases of breast cancer. However, mutations of the BRCA1 and BRCA2 genes account for up to $60 \%$ of hereditary breast cancer presentations and cause the hereditary breast/ovarian cancer syndrome (HBOCS). When a patient is carrier of pathogenic mutations in the BRCA1 gene, she has a cumulative risk (70 years) for the development of breast cancer of up to $85 \%$, and with $B R C A 2$, of up to $80 \%$. For ovarian cancer, the risk is as high as $44 \%$ with $B R C A 1$ and of $27 \%$ with BRCA2. Other associated tumors are: oropharynx, pancreas and biliary tract, colon, gastric, prostate, endometrial and skin (melanoma) cancers.

HBOCS has an autosomal dominant inheritance mechanism, and carrier patients' first-degree relatives have therefore a $50 \%$ of risk for inheriting it. ${ }^{4,5}$ It is essential for medical and paramedical personnel to identify patients at high risk for the development of hereditary breast cancer for their referral to the genetics department, where they should be inquired on hereditary or family history of cancer as part of their evaluation. The type of cancer and age at diagnosis in relatives are key to hereditary cancer syndrome integration. In some cases, there may be no history of cancer in the family, but this does not exclude the possibility of hereditary cancer syndrome.

When a patient is confirmed to be at high risk, a molecular study will be proposed according to the suspected gene/syndrome, always starting with an affected patient (if available). ${ }^{6}$ It is important for the patient to receive genetic counseling prior to undergoing a molecular study and when its results become available. Failure to obtain a comprehensive risk assessment has led to unwanted results, including wrong tests request (it should be remembered that not all cases are due to mutations in the BRCA genes), negative emotional effects, incorrect medical management guidelines and tests' wrong interpretation. This implies waste of resources (provider, time, money), late cancer diagnoses in case of false negatives and unnecessary risk-reducing surgeries owing to false positives.

On the other hand, the multi-gene panels for hereditary cancer that are offered as part of clinical services may play an important role for these patients' diagnosis; however, in the most recent update on breast and ovarian cancer familial risk assessment issued by the NCCN, the limitations of panels are mentioned: lack of knowledge on the risk level for many genes, lack of clinical guidelines and high proportion of variants of uncertain clinical significance that can be obtained when this type of studies are performed. These same guidelines specify that multi-gene panels should only be requested by a geneticist with experience on the subject for careful interpretation of the results, as well as the ensuing counseling, particularly when mutations are found in moderate-risk genes in patients with 
Table 1. Clinical characteristics of patients with suspected HBOCS.

\author{
Patients with breast cancer before 40 years of age and with at least one of the following criteria: hereditary/family history of the same \\ type of neoplasm or related neoplasm (ovary, oropharynx, pancreas and biliary tract, colon, gastric, prostate, endometrium and \\ skin [melanoma]) in two or more first or second-degree relatives. \\ Presence of multifocal or bilateral neoplasm. \\ Presence of two or more primary tumors in the same patient. \\ Breast cancer at early age and ovarian/fallopian tube cancer or peritoneal carcinomatosis in the same family branch. \\ Belonging to high-risk groups, such as Ashkenazi Jews. \\ Women $\leq 60$ years with triple-negative tumors (higher likelihood of finding mutation in BRCA1) and who express cytokeratin $5 / 6$. \\ In males: prostate cancer at early age and Gleason $\geq 7$; family history of breast cancer and/or breast cancer in males. \\ Individuals who belong to families with known mutation in susceptibility genes.
}

negative results. ${ }^{7,8} \mathrm{~A}$ laboratory with experience in the correct performance and interpretation of these tests should be chosen, owing to the risk for false positives or negatives leading to issuing erroneous recommendations to patients. This study is not a screening that can be offered to the general population, since its cost is highly elevated and the benefits for low-risk patients are limited.

Recently, mutations in BRCA1/2 that are more common in some populations, known as founder mutations, have been identified. In the Mexican population, the loss of gene BRCA1 exons 9 to 12 corresponds to approximately $10 \%$ of mutations found in this gene. However, BRCA1/2 study is still recommended to be comprehensive (sequencing and search for deletions and duplications) since there is no preferential distribution of mutations and founder mutations are only found in a percentage of the population. ${ }^{9}$

Patients should meet certain characteristics to be considered candidates to molecular study (table 1). ${ }^{4-6}$ Family and personal history allow to empirically anticipate the likelihood for a positive and informative result to be obtained. Once the molecular diagnosis is established, genetic counseling should be provided again, with particular interest in the psychological aspects a predictive test implies. A molecular test of this kind can have three types of results: positive for a deleterious mutation, negative or with variants of uncertain significance (VUS) identification. A negative result for the $B R C A$ genes does not exclude the possibility of mutations in other genes. A VUS report entails uncertainty with regard to the risk for developing cancer and the medical behavior to be followed.

Therefore, maintaining follow-up of the patient with this result is recommended, while waiting for the generation of further information on the impact of the variant on the gene's function. ${ }^{10} \mathrm{~A}$ positive result implying identification of a mutation in BRCA1 and/or BRCA2has the potential for treatment selection, follow-up, family planning and/or reproduction options personalization, as well as for risk-reduction options selection. ${ }^{11}$ The triple-negative phenotype is mainly related to mutations in BRCA1. Up to $20 \%$ of patients with this tumor histology are carriers of germline mutations and, therefore, this characteristic should be included in the diagnostic criteria, regardless of the family history (table 1).12,13

With regard to follow-up, starting breast self-exam at age 18, annual or biannual clinical examination, as well as mammography and breast MRI starting at age 25 is recommended. However, age for initiation of this can be according to earlier onset in the family. Current evidence highlights the usefulness of MRI in the diagnosis of patients with mutations in BRCA1/BRCA2, especially in those younger than 40 years, and it is therefore indicated as a part of follow-up. Other preventive options in patients that are carriers of mutations include chemoprevention with the use of tamoxifen, risk-reducing mastectomy and mastectomy/oophorectomy/salpingectomy combination. These procedures should only be considered in a group of patients carefully selected by a multidisciplinary group, based on breast cancer development objective risk, as well as on personaldesire of the patient after genetic counseling. Although molecular studies do not translate into direct benefits for the patient, extension to the family allows risk-reduction measures implementation. ${ }^{14-16}$

Pre-molecular test counseling, at any of the circumstances it is indicated for, should be accompanied by accurate information on the molecular test, its meaning and possible management of the result of the offered tests, within the legal frame of the informed consent signature, warranting each patient's autonomy. 


\section{Psycho-oncologic aspects in breast cancer}

\section{Introduction}

Psycho-oncology is a specialty that takes care of psychological, social, cultural, anthropologic, ethical-spiritual and sexuality aspects of cancer patients. ${ }^{1}$ In this context, breast cancer diagnosis has a threatening meaning for the patient and is perceived as premature death risk. This effect depends on a variety of factors such as age, socioeconomic situation, coping capability with regard to disease and the social and emotional support the patient has. ${ }^{2}$

\section{Psychological problems}

Distress is one the most prevalent psychological problems in patients with breast cancer, and it is defined by the National Cancer Comprehensive Network (NCCN) as "an unpleasant emotional experience of a psychological (cognitive, behavioral, emotional), social and/or spiritual nature that interferes with the ability to cope with cancer, its physical symptoms and/or its treatment". ${ }^{3}$

In this group of patients, distress, depression and anxiety are the most prevalent mental health problems that are closely linked to each other ${ }^{4-6}$ and are associated with sleep disorders, pain and fatigue. This is mainly observed in the subgroup of patients with metastatic cancer and there are repercussions in terms of body image and psychosocial wellbeing. ${ }^{7}$ Diagnosis and treatment of these conditions are fundamental, as well as the type of coping of the patient, since it can influence on hospital length of stay, self-care, treatment adherence and quality of life. ${ }^{8}$

Young patients (younger than 40 years) experience effects on sexuality, depressive symptoms, anxiety, body image alterations, problems with their marital relationship and for the care of their children, as well as a sense stigmatization and discrimination. ${ }^{9,10}$ Survivors of this disease may exhibit symptoms of anxiety, decreased executive function, working memory alterations and concentration problems in comparison with women with no history of cancer.

On the other hand, primary caregivers of this group of patients have been identified to be likely to suffer psychosocial repercussions such as anxiety, depression and overload. Cancer has been reported to significantly impact on couples' relationships; ${ }^{11}$ the most affected are those with poor problem-solving skills, with marital difficulties prior to the cancer diagnosis and those who differ on their perceptions and expectations with regard to cancer. ${ }^{12,13}$

\section{Assessment}

Next, four brief detection tools that can be used to identify patients and partners in need for psychosocial intervention are enumerated:

- The distress thermometer (Holland, 1999), to identify the level of emotional unrest; validated for the Mexican population by Almanza-Muñoz, Juárez and Pérez (2008)..$^{14}$

- The hospital anxiety and depression scale (HADS) (Zigmond and Snaith, 1983), to identify anxious and depressive symptoms; validated for the Mexican population by Galindo et al. (2015). ${ }^{15}$

- Zarit scale for burden assessment (1980), for patient partners who bear the role of caregivers; validated for the Mexican population by Galindo et al. (2014). ${ }^{16}$

- The dyadic adjustment scale (DAS), to assess the quality of couples' relationships; validated in the Mexican population by Moral de la Rubia (2009).17

\section{Psychological therapy}

Cognitive-behavioral therapy (CBT) is regarded as the psychological alternative for the cancer population with psychological disturbances. The purpose of this therapy is to modify cognitions and behaviors that complicate health problems by means of techniques based on scientific research; it seeks to correct patterns of thought and irrational beliefs associated with physical appearance, attractiveness and worth by improving coping resources and promoting emotional self-regulation.

CBT objectives in cancer are divided in two groups: 1) diagnosis-associated psychological problems approach, and 2) management of cancer treatment side effects such as nausea, vomiting, pain, insomnia, incontinence and sexual dysfunction.

These patients may benefit from different forms of psychological professional intervention, which can be classified as follows:

- Educational-informative interventions (counseling).

- Individual psychotherapeutic (behavioral, cognitive, dynamical) interventions.

- Interventions mediated by psychological
group-processes. ${ }^{18,19}$ 
Table 1. Evaluation and psycho-oncologic treatment

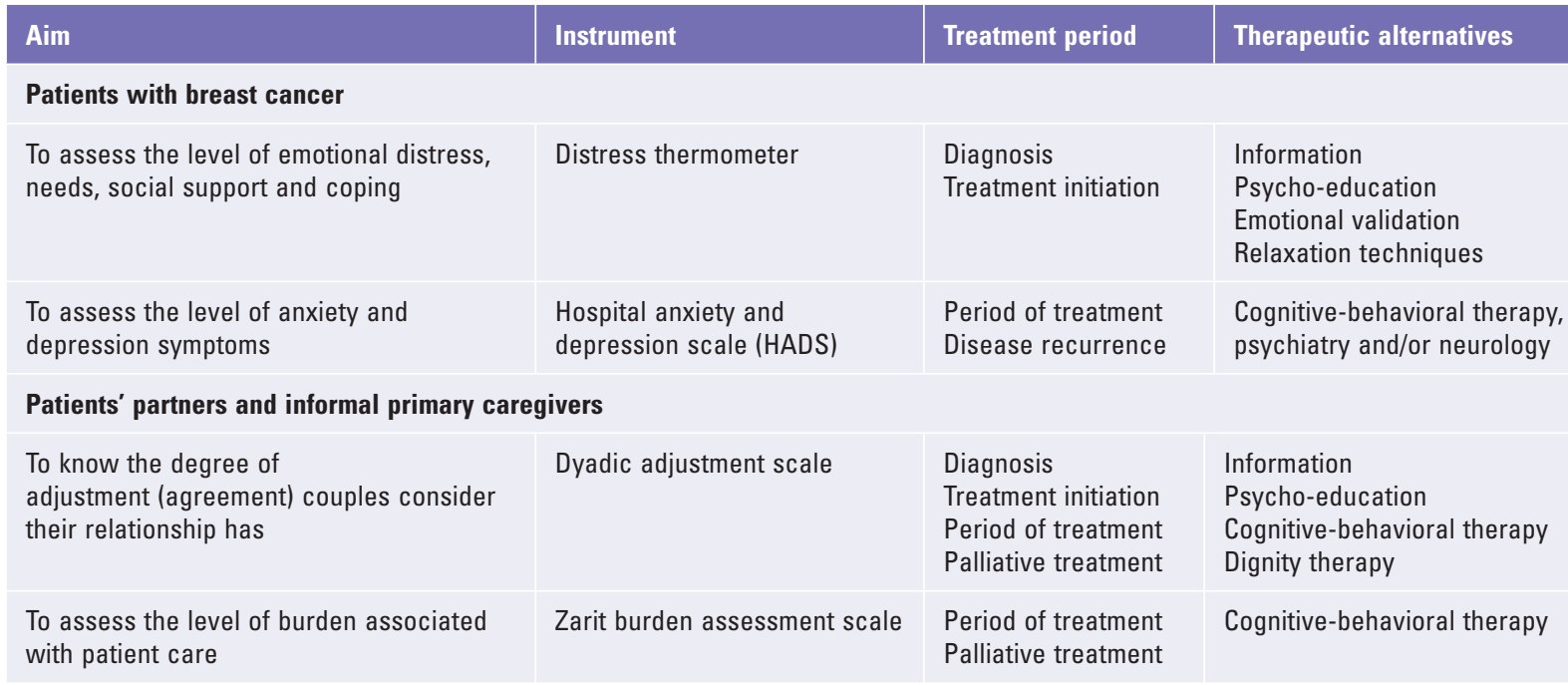

CBT modifies patterns that contribute to problems; it can also employ principles of conditioning and learning to modify problematic behaviors. ${ }^{20}$

There is sufficient evidence that cognitive-behavioral programs are efficacious to improve the control of some symptoms, affective state related to concrete situations and coping with the disease at its different phases. ${ }^{21}$ Performing further studies is recommended in order to increase the evidence in the Mexican population with regard to long-term effects and in poorly represented groups of patients (table 1). Finally, dignity therapy has shown a positive effect on emotional wellbeing in patients with advanced cancer and on palliative care.

\section{Physical rehabilitation of the patient with breast cancer}

Advances in treatments and increased survival of patients with cancer demand for rehabilitation methods to be increasingly more effective in order to achieve better quality of life for both survivors of the disease and end-stage patients.

After surgical procedures, complications may arise, some of which are exclusively related to the breast and others to the axillary lymph node dissection. Some of these complications are:

- Wound infection.

- Seromas.

- Hematomas.

- Brachial plexopathy.

- Upper limb range of motion decrease.
- Affected limb sensitivity alterations.

- Neuropathic pain.

- Lymphedema.

\section{Lymphedema}

Lymphedema is a common complication after axillary lymph node surgery for breast cancer. Currently, indicated rehabilitation is poorly known and lymphedema incidence is therefore higher than it would be if adequate prevention was practiced.

From $13 \%$ to $27 \%$ of patients with axillary dissection will develop lymphedema; ${ }^{1,2}$ the risk increases with axillary dissection extent and radiotherapy. On the other hand, overweight and obesity increase the risk for developing it in up to $80 \%$ of cases and limit treatment outcomes as well.

Lymphedema has complications such as; ${ }^{3}$

- Body image disorder.

- Situational and chronic low self-esteem.

- Risk for human dignity compromise.

- Social interaction deterioration.

- Sexual dysfunction.

- Personal identity disorder.

- Intolerance to activity.

- Self-care deficit.

\section{Lymphedema stages}

\section{Stage 0}

- No clinical data of lymphedema.

Stage I: reversible

- Evident volume increase. 
- In general, elevation of the limb reduces the swelling, but does not stop progression.

Stage II: spontaneously irreversible.

- Limb volume significantly increased.

- Elevation of the limb no longer reduces the swelling.

Stage III: lymphostatic elephantiasis

- The limb becomes exaggeratedly swollen.

- Physical impairment.

It is very important letting the patient know that lymphedema is a real and probable risk, but that it can be prevented with the right rehabilitation since the day of surgery and with adequate preventive care.

The patient should start moving the arm since the first postsurgical day: shoulder flexion and extension with the elbow bent at 90 degrees. She shouldn't make shoulder abduction movements for 7 days, since that's the time lymphatic capillaries take to restablish. ${ }^{4}$

As of the eight day, she should start arm movement with passive exercises (with help from another person) of shoulder flexion, abduction and rotation. Once full range of motion is achieved, an active exercise program should be started in order to maintain the lymphatic system permeable (www.asociacionlinfaticademexico.org).

Preventive care in the arm, chest and back on the side of surgery to decrease the risk for lymphedema are:

- Avoid efforts (carry $5 \mathrm{~kg}$ maximum).

- Avoid injuries, burns, insect bites.

- Do not sleep on the affected arm.

- Do not use jewelry or wristwatch.

- Maintain an ideal weight.

- Do not apply heat on the affected quadrant.

- Don't draw blood from the affected arm.

- Do not apply acupuncture treatments on the affected quadrant or limb.

- Use compression sleeve when travelling or doing exercise.

- Don't use diuretics, except if there is highly necessary medical indication.

If the arm increases in volume, changes its color or if its temperature rises, go see your doctor.

The preventive compression sleeve should be used to practice exercise, to travel and when doing heavy household chores.

The preventive sleeve should be special for lymphedema (compression: 20 to $30 \mathrm{mmHg}$ ) and be prescribed by a lymphedema specialist.

Indicated treatment for lymphedema is complex decongestive therapy (CDT) ${ }^{5,6}$ Although lymphedema has no cure, this treatment can reduce the swelling and keep it controlled.
The four components of CDT are:

- Thorough care of the nails and affected quadrant skin.

- Manual lymphatic drainage (MDN).

- Compression therapy with short stretch bandages or Circaid and medical compression pieces of clothing. - Lymphokinetic exercises. ${ }^{7}$

This therapy is light, non-invasive, and in most cases it restores patient control of her lymphedema and reincorporates her to a functional life.

A patient who already has lymphedema should receive training prior to using a sleeve. The use of a sleeve without training causes hand edema and makes the patient and doctor think that the sleeve does not work.

Sequential compression therapy (SCT) is a complementary part of manual lymphatic drainage (MLD), working pressure agreed between 20 and $40 \mathrm{mmHg}$ with a mean duration of 20 to 45 minutes.

It is important that all patients intervened for a tumor that may cause lymphedema receive information related to the risk for developing it and be warned on the norms for prevention and care.

Patients who require immediate physical rehabilitation: ${ }^{8}$ 1. Individuals who have undergone mastectomy, lymph node dissection and/or radiotherapy.

2. Patients with pain and/or neuropathies.

3. Patients with body mass index $>25 \mathrm{~kg} / \mathrm{m}^{2}$.

\section{Complex decongestive therapy and} physical therapy as palliative treatment in patients with tumor activity and at terminal stage

The intention of this therapy in patients with advanced disease or at terminal stage is to maintain self-sufficiency for as long as possible by preserving mobility and muscular strength and markedly reducing pain. Although lymphedema will not considerably improve, maintaining a good control of it is feasible.

\section{Palliative care in metastatic breast cancer}

Current multiple treatment options make it possible for the evolution of women with metastatic breast cancer to be prolonged, with periods where the disease can be stabilized without this meaning that other symptoms will be absent. The emphasis of oncologic treatment is focused on tumor control; however, the relief of other problems that impact on quality of life is often of secondary interest. ${ }^{1}$ 


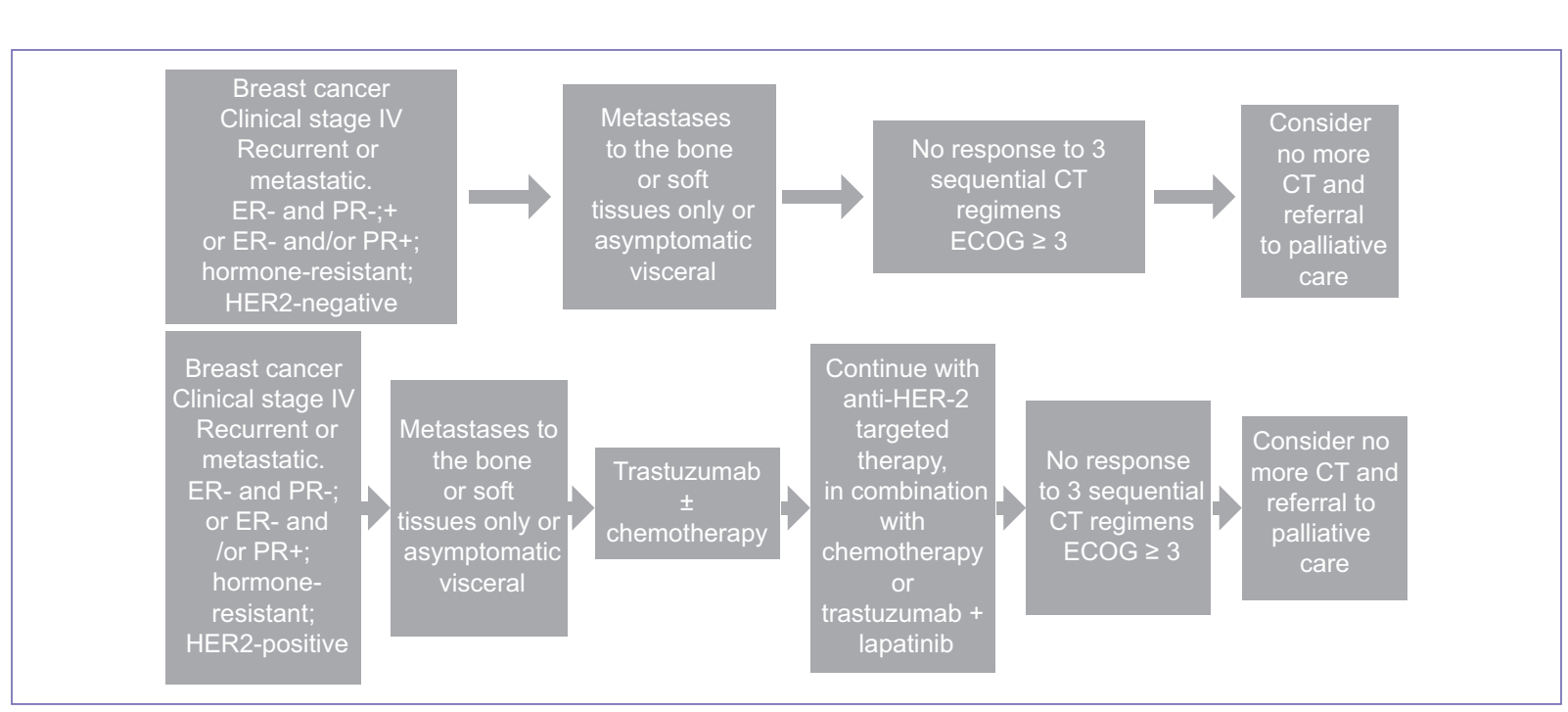

Figure 1. Criteria for referral to palliative care in patients with advanced or recurrent breast cancer. ${ }^{6}$ *Modified from Clinical practice guidelines in oncology NCCN Guidelines version 3.2015.

Palliative care is an approach intended to improve quality of life and symptom management of patients with incurable neoplastic or chronic-degenerative conditions. Its essence is to offer a comprehensive and trans-disciplinary approach to provide the patients with prevention and relief of pain, suffering and other physical, psychosocial and spiritual problems associated with their disease. ${ }^{2}$

According to multiple studies, early integration of palliative care to the management of cancer patients can improve quality of life, symptom control, satisfaction of the patient and her family, end-of-life care, survival and economic costs. ${ }^{3}$ The American Society of Clinical Oncology (ASCO) has issued a provisional recommendation to include palliative care early in oncologic management, ${ }^{4}$ which makes it clear that the oncologist plays an important role in basic palliative care as part of his/ her clinical practice.

\section{Palliative assessment by the oncologist}

Palliative assessment is carried out by means of a structured examination about the presence of pain and other symptoms. Periodical reevaluation of symptoms is important, as well as maintaining open communication with the patient and her family. According to the NCCN guidelines for palliative care, ${ }^{5}$ in the follow-up of patients with advanced or recurrent breast cancer, the oncologist should establish a routine for the detection of one or more of the following problems: symptom lack of control, distress related to cancer or its treatment, serious physical, psychological or psychiatric comorbidities, life expectancy of less than 6 months, disease progression, as well as family concerns on evolution and treatment decisions, in addition to request for palliative care by the patient or her family.

The comprehensive oncologic-palliative evaluation discussion should include the review of anti-tumor treatment risks and benefits and its prognosis, making sure that the patient and her family understand the incurable nature of the disease. In this context, the oncologist's opinion about the benefit of referral to palliative care should be considered (Fig. 1). ${ }^{6}$

\section{Symptom management by the oncologist}

Breast cancer patient symptoms are varied and changeable during the course of the disease, but they are accentuated as it progresses and at terminal phase. Pain, depression, anxiety, fatigue, dyspnea, insomnia, nausea and weight loss are common symptoms that cause increasing dependence of patients and importantly contribute to increase their suffering. Other symptoms associated with spinal cord compression, brain metastases, lymphedema and anemia negatively impact on their quality of life as well. ${ }^{5}$

\section{PAIN}

Cancer-related pain is a syndrome characterized by a constellation of signs and symptoms and is present in up to $70 \%$ of patients with advanced breast cancer owing to disease progression. ${ }^{5}$ It is important for a good clinical examination to be made in order to identify the 


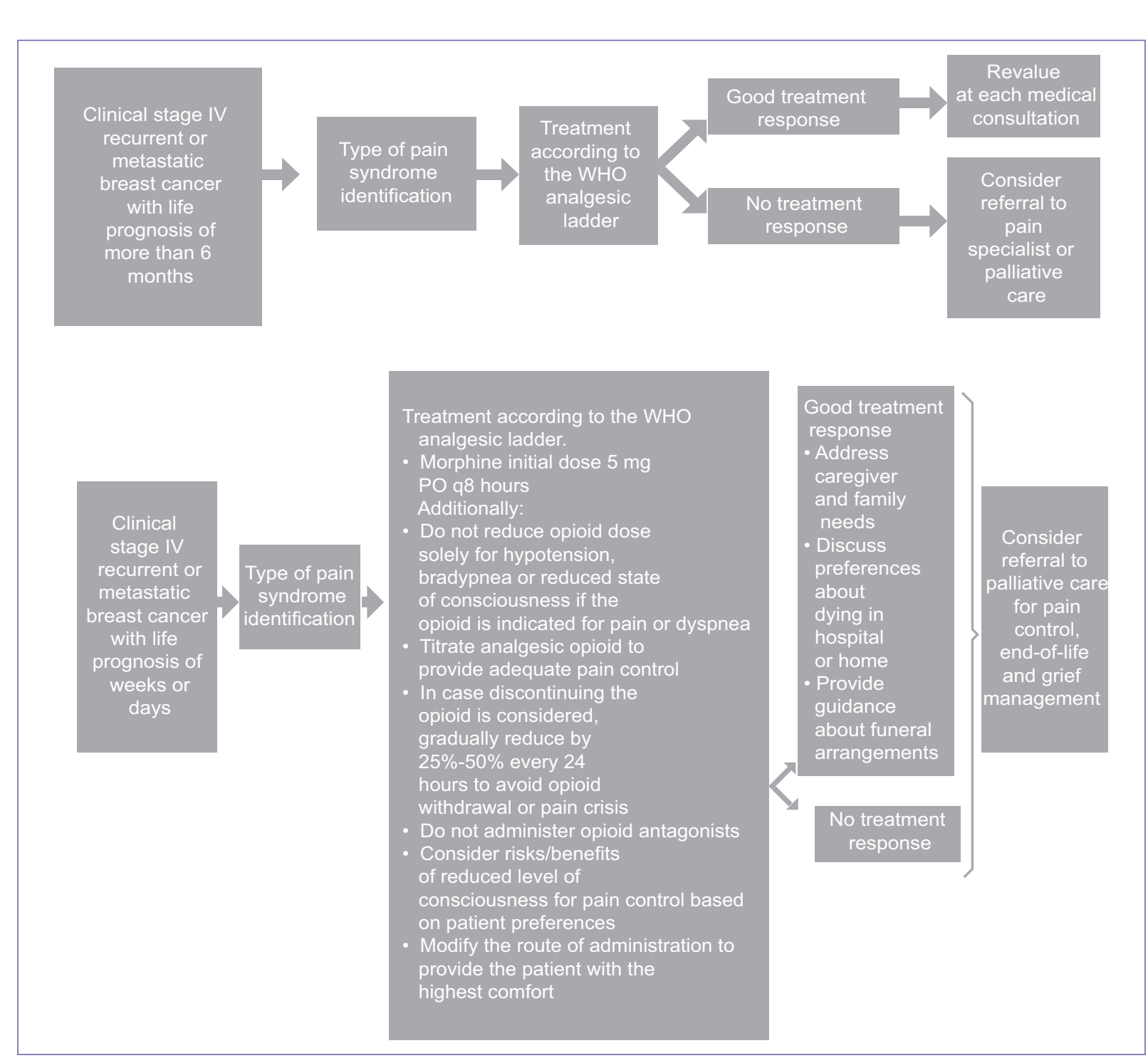

Figure 2. Pain.

*Modified from Clinical practice guidelines in oncology. Version 1.2016.

characteristics of pain, as well as psychological and social aspects that might influence on it, in addition to establishing realistic treatment goals and periodical reevaluation of the patient. Figure 2 shows some recommendations for symptom management, emphasizing on the need for consultation with pain and palliative care specialists in complex cases. ${ }^{5}$

\section{DYSPNEA}

Dyspnea is a common symptom in patients with breast cancer metastatic to the lung. The American Thoracic Society defines it as "a subjective experience of breathing discomfort of qualitatively distinct sensations that vary in intensity". Treatment of underlying causes (anemia, heart failure, asthma, pulmonary infection, etc.) should always be considered. The suggested flowchart for the management of this symptom is presented in figure $3 .^{5}$

\section{Anorexia}

Anorexia and weight loss are common in patients with advanced cancer; they contribute to the tiredness sensation of patients and are an important part of family concerns. Megestrol acetate stimulates appetite, but does not improve quality of life or muscle mass and increases the risk for edema and thromboembolic phenomena. Corticosteroids improve appetite but prolonged use causes multiple side effects. 


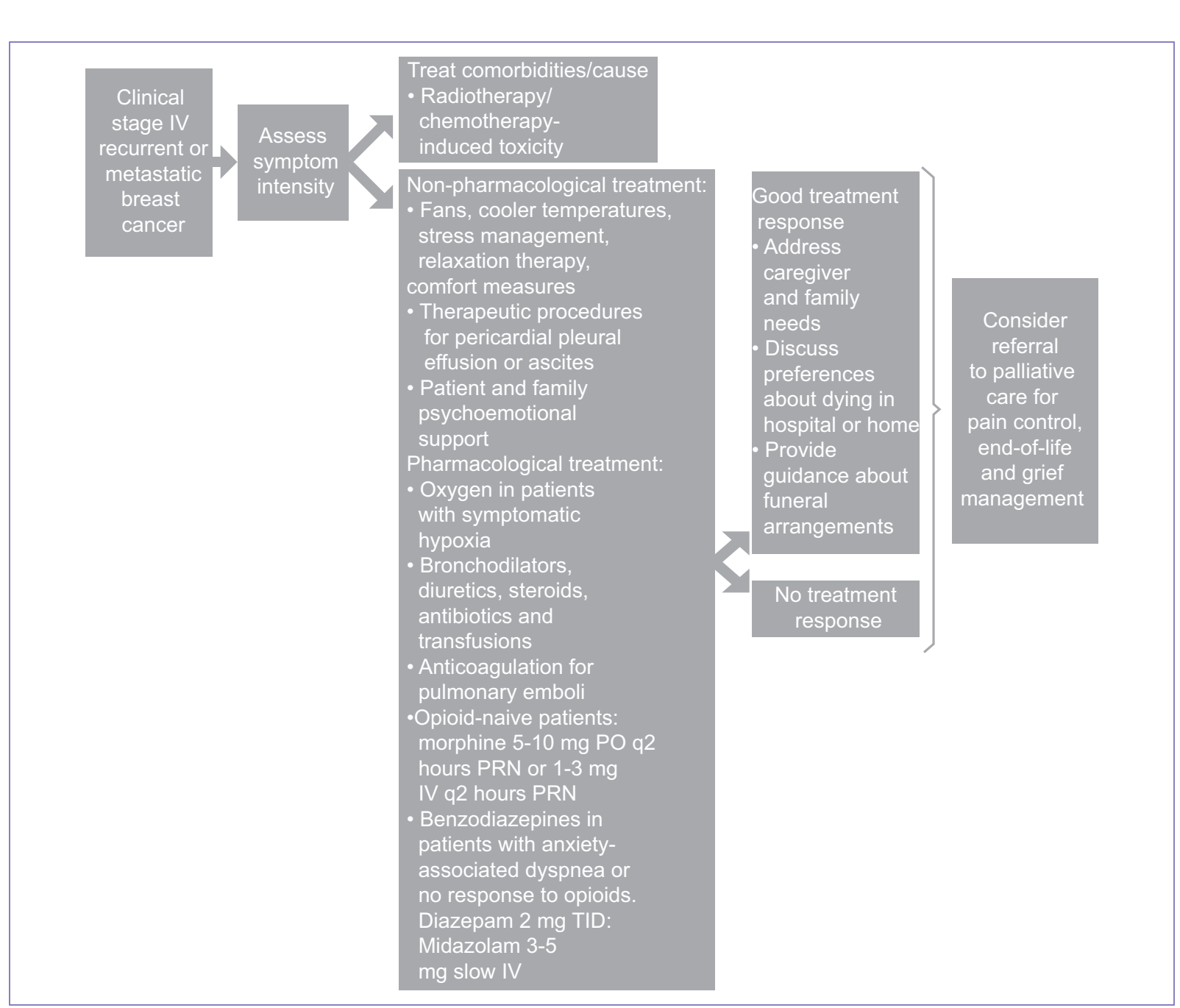

Figure 3. Dyspnea.

*Modified from Clinical practice guidelines in oncology. Version 1.2016.

\section{DELIRIUM}

Delirium is the most common neuropsychiatric complication in patients with advanced metastatic cancer. It is characterized by global brain dysfunction with fluctuations in the state of alertness, attention, thought, perception, memory, psychomotor behavior, emotions and sleep-wake cycle. Most times its etiology is multi-factorial: it can be directly caused by CNS alterations (metastasis) or by indirect effect of the disease or its treatment. Delirium can be hyperactive, hypoactive or mixed, with the latter being the most common. Hypoactive delirium is underdiagnosed in patients with advanced cancer on palliative care. There are different screening instruments for delirium assessment, the simplest of which is the Confusion Assessment Method (CAM). ${ }^{7}$

\section{Criteria for outpatient palliative care referral}

Interaction with palliative care specialists willenrich oncology practice in difficult-to-control symptoms, in some end-of-life situations and during the process of grief. Major and minor criteria have been suggested, which guide the oncologist on when to refer a patient to palliative care. Table 1 summarizes the major criteria according to an expert panel. ${ }^{8}$

The symptom palliation approach, in addition to improving breast cancer patients' quality of life, can help them and their families to have a realistic vision of short and long-term treatment goals. It can also help the oncologist to incorporate essential aspects in his/her patients' care and to accompany them through the different stages of the disease. Although the symptoms addressed in this section do not encompass the totality 
Table 1. Major criteria for palliative care referral Criteria based on patient needs

Serious physical symptoms (e.g., pain, dyspnea, nausea 7/10)

Intense emotional symptoms (depression, anxiety 7/10)

Request by the patient for hastened death

Severe emotional or spiritual distress

Guidance on advance directives and decision making

By patient request

Delirium

Brain or leptomeningeal metastases

Spinal cord compression

Criteria based on prognosis

Patient with advanced, incurable cancer diagnosis with life expectancy of less than 1 year

Advanced metastatic cancer with disease progression in spite of third-line therapy

of problems present in women with advanced breast cancer, they provide an overview on the symptom palliation approach for oncologists.

\section{REFERENCES}

\section{Introduction}

1. Primer Consenso Nacional sobre Tratamiento del Cáncer Mamario. Rev Inst. Nal Cancerol (Mex) 1995;41(3):136-145.

2. Primera Revisión del Consenso Nacional sobre Tratamiento del Cáncer Mamario. Rev Ginecol Obst Méx 2002;70:349-358.

3. Segunda Revisión del Consenso Nacional sobre el Diagnóstico y Tratamiento del Cáncer Mamario. Rev Gamo 2006;5(supl 2).

4. Tercera Revisión del Consenso Nacional sobre el Diagnóstico y Tratamiento del Cáncer Mamario. Rev Gamo 2008;7(supl 6).

5. Cuarta Revisión del Consenso Nacional sobre el Diagnóstico y Tratamiento del Cáncer Mamario. Rev Gamo 2011;10(supl 6).

6. Consenso Mexicano sobre Diagnóstico y Tratamiento del Cáncer Mamario. Quinta Revisión. Rev Gamo 2013:12(supl 3).

7. Consenso Mexicano sobre Diagnóstico y Tratamiento del Cáncer Mamario. Sexta Revisión. Rev Gamo 2015:14(supl 2).

8. Norma Oficial Mexicana NOM-041-SSA-2-2011 para la Prevención, Diagnóstico, Tratamiento, Control y Vigilancia Epidemiológica del Cáncer de Mama, México, Secretaría de Salud 2003:6-7.

\section{Epidemiology}

1. Globocan 2014, International Agency for Research on Cancer Breast Cancer Estimated Incidence, Mortality and Prevalence Worldwide in 2012.

2. Golditz G, Baer H, Tamimi R. Cancer Epidemiology and Prevention, 3rd ed, New York: Oxford Press, 2006.

3. Bray F, Piñeiros M. Cancer patterns: trends and projections in Latin America and the Caribbean: a global context. Salud Pub Mex 2016;58:104-118.

4. Ford D, Easton DF, Bishop D, et al. Risk of cancer in BRCA1-mutation carriers. Breast Cancer Linkage Consortium. Lancet 1994;343:692.

5. Gómez-Dantés H, Lamadrid-Figueroa H, Cahuana- Hurtado L, et al. The Burden of Cancer in Mexico, 1990-2013. Salud Pub Mex 2016;58:118-131.

6. Mohar-Bentacourt A, Rodríguez-Cuevas S, Barrera-Franco JL. Registro hospitalario interinstitucional de cáncer de mama, grupo de 10 mil pacientes. Presentado en el 34 a Congreso Nacional de Oncología, Tijuana, B. C., 21 de octubre de 2016.

\section{Information and education}

1. Ramos AK, Correa A. Trinidad N. Perspectives on Breast Health Education and Services Among Recent Hispanic Immigrant Women in the Midwest: a Qualitative Study in Lancaster County, Nebraska.9. Cancer Educ. Published on line July 22-2015.

2. Curbow B, Garza MA, Mc Donell K, Benz Scott L, Coyney CA. Community-based cancer screening programs in older populations: Making progress but can we do better? J Prev Med 2004;38:676-693.

3. Norma Oficial Mexicana NOM-041-SSA2-2011 para la Prevención, Diagnóstico, Tratamiento, Control y Vigilancia Epidemiológica del Cáncer de Mama, México, Secretaría de Salud.

4. Guía de práctica clínica, prevención y diagnóstico oportuno de cáncer de mama en el primer nivel de atención. Evidencias y recomendaciones. Centro Nacional de Excelencia Tecnológica en Salud (CENETEC).

5. Haines A. Identification assessment and management of overweight and obesity: summary of updated NICE guidance. BMJ 2014;349:g6608.

6. Aguilar-Cordero MJ, González-Jiménez E, García-López AP. Obesidad y su implicación en el cáncer de mama. Nutr Hosp 2011;26(4):899-903.

7. Arnold M, Pandeya N, Byrnes G, Renehan AG, et al. Global burden of cancer attributable to high body mass, index en 2012: a population based study. Lancet Oncol 2015;16(1):36-46.

8. Blair CK, Robien K, Inove-Choi, Rahn W, et al. Physical inactivity and risk of poor quality of life among elderly cancer survivors compared to women without cancer. The lowa Women's Health Study. I Cancer Surviv. Published on line may 25-2015.

9. Ortiz SP, Torres-Mejía G, Mainero $\mathrm{F}$ et al. Actividad física y cáncer de mama en mujeres mexicanas. Salud Pub Mex 2008;50(2).

10. Casla-Barrio S, Sampedro-Molinuelo J et al. Cáncer de mama y ejercicio físico. Estudio piloto. Rev Andal Med Deporte 2012;5(4):134-139.

11. Elme $A$, Utraiainen $M$ et al. Obesity and physical inactivity are related to impaired physical health of breast cancer survivors. Anticancer Res 2013;33:1595-602.

\section{Breast cancer primary prevention}

1. Fisher B, Costantino JP, Wickerham DL, et al. Tamoxifen for the prevention of breast cancer: current status of the National Surgical Adjuvant Breast and Bowel Project P-1 study. J Natl Cancer Inst 2005:97(22):1652-1962.

2. King MC, Wieand S, Hale K, Lee M, et al. Tamoxifen and breast cancer incidence among women with inherited mutations in BRCA 1 and BRCA. National Surgical Adjuvant Breast and Bowel Project (NSABP-P1) Breast Cancer Prevention Trial. JAMA 2001;286(18):2251-2256.

3. Familial breast cancer: classification, care and managing breast cancer related risks in people with a family history of breast cancer. Clinical Guideline Published: 25 June 2013. nice.org.uk/guidance/cg164.

4. Visvanathan K, Hurley $P$, Bantug $E$, et al. Use of pharmacologic intervention for breast cancer risk reduction: American Society of Clinical Oncology Clinical Practice Guideline. J Clin Oncol 2013:31(23):2942-2962.

5. Invasive Breast Cancer 2017, NCCN Clinical Practise Guidelines in Oncology

6. Nelson HD, Smtih B, Griffin J, and Rongwel Fu. Use of medications to reduce risk for primary breast cancer: A systematic review for the U.S. Preventive Services Task Force. Ann Intern Med 2013;158:604-614.

7. Barrer-Connor E, Mosca L, Collins P, Geiger M, et al. Effects of raloxifene of cardiovascular events and breast cancer in postmenopausal women for the Raloxifene Use for the Heart (RUTH) Trial Investigators. N Eng J Med 2006;355:125-37.

8. Ettinger B, Black DM, Mitlak BH, et al. Reduction of vertebral fracture risk in postmesopausal women with osteoporosis treated with raloxifene. Results from a 3 year randomized clinical trial. JAMA 1999:282(7):637-645.

9. Martino S, Cauley J, Barret-Connor E, Powles T, Mershon J. Continuing outcomes relevant to Evista: breast cancer incidence in postmenopausal osteoporotic women in a randomized trial of raloxifene. J Natl Cancer Inst 2004;96(23):1751-1761.

10. Vogel V, Costantino J, Wickerham L, Cronin W, et al. Update of the National Surgical Adjuvant Breast and Bowel Project Study of Tamoxifen and Raloxifene (STAR) P-2 Trial: Preventing Breast Cancer. Cancer Prev Res 2010;3(6):696-706

11. Goss P, Ingle J, Alés-Martínez J, Cheung A, et al. Exemestane for breast-cancer prevention in postmeopusal women. $\mathrm{N}$ Engl $\mathrm{J}$ Med 2011;364(25):2381-2391.

12. Cuzick J, Sestak I, Forbes J, Dowsett M, Knox J, at al. Anastrozole for prevention of breast cancer in high -risk postmenopausal women (IBIS-II) an international, doble blind, randomised placebo-controlled trial. Lancet 2014;383:1041-48.

\section{Early diagnosis. Breast assessment by imaging}

1. Nelson HD, Tyne K, Naik A, et al. Screening for breast cancer: an update for the U.S. Preventive Services Task Force. Ann Intern Med 2009:151(10):727-737, W237-42. 
2. Miller $A B$. Conundrums in screening for cancer. Mini review. Int J Cancer 2010;126:1039-1046.

3. Kerlikowske K. Efficacy of screening mammography among women aged 40 to 49 years and 50 to 69 years: comparison of relative and absolute benefit. J Natl Cancer Inst Monogr 1997;(22):79-86.

4. Welch HG. Over-diagnosis and mammography screening. J Natl Cancer Inst 2010;102:605-613.

5. Ronckers CM, Erdmann CA, Land CE. Radiation and breast cancer: a review of current evidence. Breast Cancer Res 2005;7(1):21-32.

6. Pisano ED, Gatsonis C, Hendrick E, et al. Diagnostic performance of digital versus film mammography for breast-cancer screening. $\mathrm{N}$ Engl J Med 2005;353(17):1773-1783.

7. Fenton JJ, Xing G, Elmore JG, et al. Short-term outcomes of screening mammography using computer-aided detection: a population-based study of medicare enrollees. Ann Intern Med 2013;158(8):580-587.

8. Gilbert FJ, Tucker L, Gillan MG, et al. TOMMY trial: a comparison of TOMosynthesis with digital MammographY in the UK NHS Breast Screening Programme-a multicentre retrospective reading study comparing the diagnostic performance of digital breast tomosynthesis and digita mammography with digital mammography alone. Health Technol Assess 2015;19(4):i-xxv.

9. Hooley RJ, Scoutt LM, Philpotts LE. Breast Ultrasound. State of the ART. Radiology 2013;268.

10. Stafford RJ, Whitman GJ. Ultrasound physics and technology in breast imaging. Ultrasound Clin 2011;6(3):299-312

11. Raza S, Chikarmane SA, Neilsen SS, et al. BI-RADS 3, 4, and 5 lesions: value of US in management follow-up and outcome. Radiology 2008:248(3):773-781.

12. Moon WK, Noh DY, Im JG. Multifocal, multicentric, and contralateral breast cancers: bilateral whole-breast US in the preoperative evaluation of patients. Radiology 2002;224(2):569-576.

13. Bream R, Lenihan M, Lieberman J, et al, Screening breast ultrasound: Past, present, and future. AJR 2015;204:234-240.

14. Ecanow JS, Abe $\mathrm{H}$, et al. Axillary staging of breast cancer: what the radiologist should know. Radiographics 2013;33:1589-1612.

15. Rosen PP. Pathologic examination of breast and lymph node specimens In: Rosen's breast pathology, 3rd ed. Philadelphia: Lippincott, Williams \& Wilkins, 2009, 1077-1089.

16. Akıncı M, Bulut SP, Erözgen F, et al. Predictive value of fine needle aspiration biopsy of axillary lymph nodes in preoperative breast cancer staging. Ulus Cerrahi Derg 2016;32:191-196.

17. Lee $\mathrm{CH}$, Dershaw DD, Kopans D, et al. Breast cancer screening with imaging: recommendations from the Society of Breast Imaging and the ACR on the use of mammography, breast MRI, breast ultrasound, and other technologies for the detection of clinically occult breast cancer. $J$ Am Coll Radiol 2010;7:18-27.

18. Jansen SA, Shimauchi A, Zak L, et al. Kinetic curves of malignant lesions are not consistent across MRI systems: need for improved standardization of breast dynamic contrast-enhanced MRI acquisition. AJR 2009;193:832-839.

19. Agrawal G, Su MY, Nalcioglu $O$, et al. Significance of breast lesion descriptors in the ACR BI-RADS lexicon. Cancer 2009;115:1363-1380.

20. Abramson RG, Arlinghaus LR, Weis JA, et al. Current and emerging quantitative magnetic resonance imaging methods for assessing and predicting the response of breast cancer to neoadjuvant therapy. Breast Cancer 2012;2012(4):139-154.

21. Brasic N, Wisner DJ, Joe BN. Breast MR imaging for extent of disease assessment in patients with newly diagnosed breast cancer. Magn Reson Imaging Clin N Am 2013;21(3):519-532.

\section{Non-palpable suspicious lesion}

1. Norma Oficial Mexicana NOM-041-SSA2-2011 para la prevención, diagnóstico, tratamiento, control y vigilancia epidemiológica del cáncer de mama, Secretaría de Salud, México.

2. Bruening W, Fontanarosa J, Tipton K, et al. Systematic review: comparative effectiveness of core-needle and open surgical biopsy to diagnose breast lesions. Ann Intern Med 2010;152(4):238-246.

3. Ciatto S, Houssami N, Ambrogetti D, et al. Accuracy and underestimation of malignancy of breast core needle biopsy: the Florence experience of over 4000 consecutive biopsies. Breast Cancer Res Treat 2007:101;3:291-297.

\section{Histopathological study}

1. Morrow M, Van Zee KJ, Solin LJ, et al. Society of Surgical Oncology-American Society for Radiation Oncology, American Society of Clinical Oncology Consensus Guideline of margins of breast-conserving with whole breast irradiation in ductal carcinoma in situ. J Clin Oncol 2016;34:4040-4046
2. Lester S, Bose S, et al. Protocol for the examination of specimens from patients with invasive carcinoma of the breast. AJCC/UICC TNM 7th Ed. January 2016.

3. Lakhani SR, Ellis IO, Schnitt SJ, Tan PH and van de Vijver MJ (eds). WHO Classification of Tumours of the Breast, IARC, Lyon, 2012.

4. Le Doussal V, Tubiana HM, Friedman S, et al. Prognostic value of histologic grade nuclear components of Scarff-Bloom-Richardson (SBR). An improved score modification based on a multivariable analysis of 1262 invasive ductal breast carcinomas. Cancer 1989;64:1914-1921.

5. Salgado R, Denkert C, Demaria S, et al. The evaluation of tumor infiltrating lymphocytes (TILs) in breast cancer: recommendations by an International TILS Working Group 2014. Ann Oncol 2015;26:259-271.

6. Symmans WF, Peintinger F, Hatzis $C$, et al. Measurement of residual breast cancer burden to predict survival after neoadjuvant chemotherapy. $\mathrm{J}$ Clin Oncol 2007:25:4414-4422.

7. Ueng SH, Mezzeti H, Tavassoli FA. Papillary neoplasms of the breast. Arch Pathol Lab Med 2009;133:893-907.

8. Rosen PP. Columnar cell hyperplasia is associated with lobular carcinoma in situ and tubular carcinoma. Am J Surg Pathol 1999;23:1561.

9. Tarek MA, Abdel-Fatah, Powe AG. High frequency of coexistence of columnar cell lesions, lobular neoplasia and low grade ductal carcinoma in situ with invasive tubular carcinoma and invasive lobular carcinoma. Am J Surg Pathol 2007;31:417-426.

10. Zhong F, Rui Bi, Yu B, Cheng Y. Carcinoma arising in microglandular adenosis of the breast: triple negative phenotype with variable morphology. Int J Clin Exp Pathol 2014;7(9):6149-6156.

11. Salarieh, Sneige N. Breast carcinoma arising in microglandular adenosis. A review of literature. Arch Pathol Lab Med 2007:131:1397-1399.

12. Lester SC, Bose S, Chen YY, et al. Protocol for the examination of specimens from patients with ductal carcinoma in situ of the breast. Arch Pathol Lab Med 2009;133(1):15-25.

13. Dadmanesh F, Fan X, Dastane A, et al. Comparative analysis of size estimation by mapping and counting number of blocks with ductal carcinoma in situ in breast excision specimens. Arch Pathol Lab Med 2009;133:26-30.

14. Grin A, Horne G, Ennis M, O'Malley FP. Measuring extent of ductal carcinoma in situ in breast excision specimens. A comparison of four methods. Arch Pathol Lab Med 2009;133:31-37.

15. Silverstein MJ, Lagios MD, Craig PH, et al. A prognostic index for ductal carcinoma in situ of the breast. Cancer 1996;77:2267-2274.

16. The Consensus Conference Committee. Consensus conference on the classification of ductal carcinoma in situ. Cancer 1997;80:1798-1802.

17. Silverstein MJ. The University of Southern California/Van Nuys prognostic index for ductal carcinoma in situ of the breast. Am J Surg 2003;186:337-343.

18. Edge SB, Compton CC. The American Joint Committee on Cancer: the 7th edition of the AJCC cancer staging manual and the future of TNM. Ann Surg Oncol 2010;17:1471-1474.

19. Motomura K, Nagumo S, Komoike Y, et al. Intraoperative imprint cytology for the diagnosis of sentinel node metastases in breast cancer. Breast Cancer 2007; 14:350-353.

20. Pérez-Sánchez VM, Vela-Chávez TA, Villarreal-Colin P. Intraoperative touch imprint cytology of sentinel lymph nodes in breast cancer: experience at a tertiary care center in Mexico. Med Oncol 2010;27(2):233-236.

21. Cserni G. Pathological evaluation of sentinel lymph nodes. Surg Oncol Clin N Am 2007;16:17-34.

22. Reintgen $M$, Kerivan $L$, et al. Breast lymphatic mapping and sentinel lymph node biopsy. Clin Breast Cancer 2016;16(3):155-165.

23. Hammond ME, Hayes DF, Dowsett M, et al. American Society of Clinical Oncology/College of American Pathologists guideline recommendations for immunohistochemical testing of estrogen and progesterone receptors in breast cancer. Arch Pathol Lab Med 2010;134:907-922.

24. Wolff AC, Hammond ME, Schwartz JN, et al. American Society of Clinical Oncology/College of American Pathologists guideline recommendations for human epidermal growth factor receptor 2 testing in breast cancer. $J$ Clin Oncol 2007;25:118-145.

25. Gown AM. Current issues in ER and HER2 testing by $\mathrm{IHC}$ in breast cancer. Mod Pathol 2008;21:S8-S15.

26. McCarty KS, Jr., Miller L, et al. Estrogen receptor analyses. Correlation of biochemical and immunohistochemical methods using monoclonal antireceptor antibodies. Arch Pathol Lab Med 1985;109:716-721.

27. Allred DC, Harvey JM, Berardo M, et al. Prognostic and predictive factors in breast cancer by immunohistochemical analysis. Mod Pathol 1998;11:155-168.

28. Kos S, Dabbs DJ. Biomarker assessment and molecular testing for prognostication in breast cancer. Histopathology 2016;68:70-85.

29. Wolff AC, Hammond EH, Hicks DG, et al. Recommendations for human epidermal growth factor receptor 2 testing in breast cancer: American Society of Clinical Oncology/College of American Pathologists clinical practice guideline update. Arch Pathol Lab Med 2014;138:241-256.

30. Wolff AC, Hammond EH, Hicks DG, et al. Recommendations for human epidermal growth factor receptor 2 testing in breast cancer: American Society of Clinical Oncology/College of American Pathologists clinical practice guideline update. J Clin Oncol 2013;31(31):3997-4013. 
31. Dowsett M, Nielsen T. Assessment of Ki67 in breast cancer: Recommendations from the International Ki67 in Breast Cancer Working Group. J Natl Cancer Inst 2011;103:1656-1664.

32. Penault-Llorca F, Radosevic-Robin N. Ki67 assessment in breast cancer: an update. Pathology 2017;49(2):166-171. doi: 10.1016/j.pathol.2016.11.006

33. Jang MH, Kim HJ, et al. A comparison of Ki 67 counting methods in luminal Breast Cancer: The Average Method vs. the Hot Spot Method. PLoS One 2017;12(2):e0172031.

34. Brown R. Quality management in immunohistochemistry. In: Quality management in anatomic pathology: promoting patient safety through systems improvement and error reduction. The College of American Pathologists 2005;93-110.

35. Arnould L, Roger P, Mac Grogan G, et al. Accuracy of Her2 status determination on breast core-needle biopsies (immunohistochemistry, FISH,CISH and SISH vs FISH). Mod Pathol 2012;25:675-682.

36. Perou CM, Sorlie T, Eisen MB, et al. Molecular portraits of human breas tumors. Nature 2000:406(6797):747-752.

37. Rakha EA, El-Sayed ME, Reis-Filho JS, Ellis IO. Expression profiling technology: its contribution to our understanding of breast cancer. Histopathology 2008:52:67-81.

38. Marchio C, Reis-Filho JS. Molecular diagnosis in breast cancer. Diagnostic Histopathology 2008;14(5):202-213.

39. Geyer FC, Marchio C, Reiss-Filho JS. The role of molecular analysis in breast cancer. Pathology 2009;41:77-88.

49. Barghava R, Striebel J, Beriwal S, et al. Prevalence, morphologic features and proliferation index of breast carcinoma molecular classes using inmunohistochemical surrogate markers. Int J Clin Exp Pathol 2009;2:444-455.

41. Lara-Medina F, Pérez-Sánchez V, Saavedra-Pérez D, et al. Triple-negative breast cancer in Hispanic patients. High prevalence, poor prognosis, and association with menopausal status, body mass index and parity. Cancer 2011:117:3658-3669.

42. Robles-Castillo J, Ruvalcaba-Limón E, Maffuz A, Rodríguez-Cuevas S Cáncer de mama en mujeres mexicanas menores de 40 años. Ginecol Obstet Mex 2011;79(8):482-488.

43. Goldhirsch A, Winer EP, et al. Personalizing the treatment of women with early breast cancer: highlights of the St Gallen International Expert Consensus of the Primary Therapy of Early Breast Cancer 2013. Ann Oncol 2013; 24(9):2206-2223.

44. Coates AS, Winer EP, et al. Personalizing the treatment of women with early breast cancer: highlights of the St Gallen International Expert Consensus of the Primary Therapy of Early Breast Cancer 2015. Ann Oncol 2015; 26(8):1533-1546.

45. Senkus E, Kyriades S. Primary breast cancer: ESMO Clinical Practice Guidelines for diagnosis, treatment and follow up. Ann Oncol 2015;26(su$\mathrm{ppL} 5): \mathrm{v} 8-\mathrm{v} 30$.

46. Bertucci F, Finetti $P$, Cervera N, et al. How basal are triple negative breast cancers? Int J Cancer 2008;123(1):236-240.

47. Nielsen TO, Hse FD, Jensen K, et al. Inmunohistochemical and clinical characterization of the basal-like subtype of invasive breast carcinoma Clin Can Res 2004:10:5367-5374.

48. Perou CM. Molecular stratification of triple-negative breast cancers. Oncologist 2011;16(suppl 1):61-70.

49. Lehmann BD, Bauer JA, Chen X, et al. Identification of human triple-negative breast cancer subtypes and preclinical models for selection of targeted therapies. J Clin Invest 2011;121:2750-2767.

50. Weigelt B, Geyer FC, Reis-Filho JS. Histological types of breast cancer How special are they? Mol Oncol 2010;4:192-208.

51. Cadoo KA, McArdle O, O'Shea AM, et al. Management of unusual histological types of breast cancer. Oncologist 2012;17(9):1135-1145.

52. Christgen $M$, Steinemann D. Lobular breast cancer. Clinical, molecular and morphological characteristics. Pathol Res Pract 2016; 212(7):583-597.

53. Sgroi DC. Preinvasive breast cancer. Annu Rev Pathol Mech Dis 2010;5:193-221.

54. Lopez-Garcia MA, Geyer FC, Lacroix-Triki M, et al. Breast cancer precursors revisited: molecular features and progression pathways. Histopathology 2010;57(2):171-192.

55. Gradishar WJ, Anderson BO, Balassanian R, et al. Invasive Breast Cancer Version 1.2016, NCCN Clinical Practice Guidelines in Oncology. J Natl Compr Canc Netw. 2016;14(3):324-354.

56. Senkus E, Kyriakides S, Ohno S, et al. Primary breast cancer: ESMO Clinical Practice Guidelines for diagnosis, treatment and follow-up. Ann Oncol. 2015;26(Suppl 5):8-30.

57. Harris LN, Ismaila N, McShane LM, et al. Use of Biomarkers to Guide Decisions on Adjuvant Systemic Therapy for Women With Early-Stage Invasive Breast Cancer: American Society of Clinical Oncology Clinical Practice Guideline. J Clin Oncol. 2016;34(10):1134-1150.

58. Coates AS, Winer EP, Goldhirsch A, et al. Tailoring therapies--improving the management of early breast cancer: St Gallen International Expert Consensus on the Primary Therapy of Early Breast Cancer 2015. Ann Oncol. 2015;26(8):1533-1546.

59. Paik S, Shak S, Tang G, et al. A multigene assay to predict recurrence of tamoxifen-treated, node-negative breast cancer. The New England journal of medicine. 2004;351(27):2817-2826.
60. Paik S, Tang G, Shak S, et al. Gene expression and benefit of chemotherapy in women with node-negative, estrogen receptor-positive breast cancer. Journal of clinical oncology : official journal of the American Society of Clinical Oncology. 2006;24(23):3726-3734.

61. Sparano JA, Gray RJ, Makower DF, et al. Prospective Validation of a 21-Gene Expression Assay in Breast Cancer. N Engl J Med. 2015;373(21):2005-2014.

62. van de Vijver MJ, He YD, van't Veer LJ, et al. A gene-expression signature as a predictor of survival in breast cancer. The New England journal of medicine. 2002;347(25):1999-2009.

63. Mittempergher L, de Ronde JJ, Nieuwland M, et al. Gene expression profiles from formalin fixed paraffin embedded breast cancer tissue are largely comparable to fresh frozen matched tissue. PloS one. 2011;6(2):e17163.

64. Sapino A, Roepman P, Linn SC, et al. MammaPrint molecular diagnostics on formalin-fixed, paraffin-embedded tissue. The Journal of molecular diagnostics: JMD. 2014;16(2):190-197.

65. Cardoso F, van't Veer LJ, Bogaerts J, et al. 70-Gene Signature as an Aid to Treatment Decisions in Early-Stage Breast Cancer. N Engl J Med. 2016:375(8):717-729.

66. Filipits M, Rudas M, Jakesz R, et al. A new molecular predictor of distant recurrence in ER-positive, HER2-negative breast cancer adds independent information to conventional clinical risk factors. Clin Cancer Res. 2011;17(18):6012-6020.

67. Dubsky P, Filipits M, Jakesz R, et al. EndoPredict improves the prognostic classification derived from common clinical guidelines in ER-positive, HER2-negative early breast cancer. Ann Oncol. 2013;24(3):640-647.

68. Martin M, Brase JC, Calvo L, et al. Clinical validation of the EndoPredict test in node-positive, chemotherapy-treated ER+/HER2- breast cancer patients: results from the GEICAM 9906 trial. Breast Cancer Res. 2014:16(2):R38

69. Genomic Health, Breast \& Colon Pathology Guidelines. http://breastcancer.oncotypedx.com/en-US/Professional Invasive/Ordering/ /media/ Files/ ODX-Breast/GHI020-INTPathologyReport.pdf.

\section{TNM classification}

1. Edge SB, Byrd DR, Compton CC, et al. AJCC cancer staging manual. 7th ed. New York, NY: Springer, 2010:347-376.

2. Giuliano A, Connolly JL, Edge SB, et al. Breast cancer-major changes in the American Joint Committee on Cancer eighth edition cancer staging manual. CA Cancer J Clin 2017 Mar 14. doi: 10.3322/caac.21393.

\section{Breast cancer treatment and interdisciplinary management}

1. Unger-Saldana $K$, et al. Health delay and its effect on clinical stage of breast cancer: Multicenter study. Cancer 2015;121(13):2198-2206.

\section{Carcinoma in situ}

1. Morrow M, Van Zee KJ, Solin LJ, et al. Society of Surgical Oncology-American Society for Radiation Oncology-American Society of Clinical Oncology Consensus Guideline on Margins for Breast-Conserving Surgery with Whole Breast Irradiation in Ductal Carcinoma In Situ. Ann Surg Oncol 2016;23(12):3801-3810.

2. Wang SY, Chu H, Shamliyan T, et al. Network meta-analysis of margin threshold for women with ductal carcinoma in situ. J Natl Cancer Inst 2012;104(7):507-516

3. Badruddoja M. Ductal carcinoma in situ of the breast: a surgical perspective. Int J Surg Oncol 2012;2012:761364. doi:10.1155/2012/761364.

4. Skandarajah AR, Mann B. Selective use whole breast radiotherapy after breast conserving surgery for invasive breast cancer and DCIS. Surgeon 2013:11(5):278-285

5. Cutuli $\mathrm{B}$, et al. Radiotherapy in DCIS, an underestimated benefit? Radiother Oncol 2014;112:1-8

6. Allred D, Bryant J, Land S, et al. Estrogen receptor expression as a predictive marker of the effectiveness of tamoxifen in the treatment of DCIS: findings from NSABP protocol B-24. Breast Cancer Res Treat 2002;76(suppl 1):S36[A30]

7. Goss PE, Ingle JN Alés-Martínez JE, et al. Exemestane for breast-cancer prevention in postmenopausal women. N Engl J Med 2011;364:2381-2391.

8. Di Saverio S, Catena F, Santini D, et al. 259 patients with DCIS of the breast applying USC/Van Nuys prognostic index: a retrospective review with long term follow up. Breast Cancer Res Treat 2008;109(3):405-416.

\section{Surgical treatment modalities at stages I and II}

\section{Conservative and radical surgery}

1. Toth BA, Lappert P. Modified skin incisions for mastectomy: the need for plastic surgical input in preoperative planning. Plast Reconstr Surg 1991:87:1048-1053. 
2. Simmons RM, Adamovich TL. Skin-sparing mastectomy. Surg Clin North Am 2003;83:885-899.

3. Lanitis S, Tekkis PP, Sgourakis G, et al. Comparison of skin-sparing mastectomy versus non-skin-sparing mastectomy for breast cancer: a meta-analysis of observational studies. Ann Surg 2010;251:632-639.

4. Crowe JP Jr, Kim JA, Yetman R, et al. Nipple-sparing mastectomy: technique and results of 54 procedures. Arch Surg 2004:139:148-150.

5. Alm EI-Din MA, Taghian AG. Breast conservation therapy for patients with locally advanced breast cancer. Semin Radiat Oncol 2009;19:229-235.

6. Sabel MS. Surgical considerations in early-stage breast cancer: lessons learned and future directions. Semin Radiat Oncol 2011;21:10-19.

7. Moran MS, Schnitt SJ, Giuliano AE, et al. Society of Surgical Oncology-American Society for Radiation Oncology consensus guideline on margins for breast-conserving surgery with whole-breast irradiation in stages I and II invasive breast cancer. Ann Surg Oncol 2014;21(3):704-716.

8. Moran MS, Schnitt SJ, Giuliano AE, et al. Society of Surgical Oncology-American Society for Radiation Oncology consensus guideline on margins for breast-conserving surgery with whole-breast irradiation in stages I and II invasive breast cancer. Int J Radiat Oncol Biol Phys 2014;88:553-564.

9. Wijayanayagam A, Kumar AS, Foster RD, Esserman LJ. Optimizing the total skin-sparing mastectomy. Arch Surg 2008;143:38-45.

\section{Oncoplastic surgery}

1. Clough KB, Kaufman GJ, Nos C, et al. Improving breast cancer surgery: A classification and quadrant per quadrant atlas for oncoplastic surgery. Ann Surg Oncol 2010;17:1375-1391.

2. Giacalone PL, Roger $\mathrm{P}$, Dubon $\mathrm{O}$ et al. Comparative study of the accuracy of breast reconstruction in oncoplastic surgery and quadrantectomy in breast cancer. Ann Surg Oncol 2007;14(2):605-614.

3. Rietjens M, Urban CA, Petit JY, et al. Long-term oncologic results of breast conservative treatment with oncoplastic surgery. Breast 2007;16(4):387-395.

4. Acea B. Técnicas oncoplásticas en el tratamiento quirúrgico del cáncer de mama, $2^{\mathrm{a}}$ edición. Elsevier Masson, 2009.

5. Staub G, Fitoussi A, Falcou MC, Salmon RJ. Breast cancer surgery: use of mammoplasty. Results. Series of 298 cases. Ann Chir Plast Esthet 2007:53(2):124-134

6. Jatoi I, Kaufmann M, Petit JY. Atlas of breast surgery. Berlin-Heidelberg: Springer-Verlag eds., 2006.

7. Andree C, Farhadi J, Goosens D, et al. A position statement on optimizing the role of oncoplastic breast surgery. Eplasty 2012;12:e40.

8. Eaton BR, Losken A, Okwan-Duodu D, et al. Local recurrence patterns in breast cancer patients treated with oncoplastic reduction mammoplasty and radiotherapy. Ann Surg Oncol 2014;21:93-99.

9. Pezner RD, Tan MC, Clancy SL, et al. Radiation therapy for breast cancer patients who undergo oncoplastic surgery. Am J Clin Oncol 2013;36:535-539.

10. Schaverien MV, Stallard S, Dodwell D, Doughty JC. Use of boost radiotherapy in oncoplastic breast-conserving surgery. A systematic review. EJSO 2013:39:1179-1185.

\section{Surgical treatment of axilla}

1. Lyman GH, Giuliano AE, Somerfield MR, et al. American Society of Clinical Oncology guideline recommendations for sentinel node biopsy in early-stage breast cancer. J Clin Oncol 2005;23:7703-7720.

2. Cox CE. Lymphatic mapping in breast cancer: combination technique Ann Surg Oncol 2001;8:67S-70S.

3. Krag DN, Anderson SJ, Julian TB, et al. Sentinel-lymph node resection compared with conventional axillary-lymph node dissection in clinically node-negative patients with breast cancer: overall survival findings from the NSABP B-32 randomised phase 3 trial. Lancet Oncol 2010;11:927-933.

4. Kuehn, Vogl FD, Helms G, et al. Sentinel-node biopsy for axillary staging in breast cancer: results from a large prospective German multi-institutional trial. Eur J Surg Oncol 2004;30:252-259.

5. Veronesi U, Paganelli G, Viale G, et al. A randomized comparison of sentinel node biopsy with routine axillary dissection in breast cancer. $\mathrm{N}$ Eng J Med 2003;349:546-553.

6. Mansel RE, Fallowfield L, Kissin M, et al. Randomized multicenter trial of sentinel node biopsy versus standard axillary treatment in operable breast cancer: the ALMANAC trial. J Natl Cancer Inst 2006;98:599-609.

7. Kargozaran H, Shah M, Li Y, et al. Concordance of peritumoral technetium $99 \mathrm{~m}$ colloid and subareolar blue dye injection in breast cancer sentinel lymph node biopsy. J Surg Res 2007;143:126-129.

8. Meyer-Rochow GY, Martin RC, Harman CR. Sentinel node biopsy in breast cancer: validation study and comparison of blue dye alone with triple modality localization. ANZ J Surg 2003;73:815-818.
9. Krikanova M, Biggar M, Moss D, et al. Accuracy of sentinel node biopsy for breast cancer using blue dye alone. Breast J 2010;16:384-388.

10. DuPont E, Cox C, Shivers S, et al. Learning curves and breast cancer lymphatic zapping: institutional volume index. J Surg Res 2001;97:92-96.

11. Giuliano AE, Hunt KK, Ballman KV, et al. Axillary dissection vs no axillary dissection in women with invasive breast cancer and sentinel node metastases: a randomized clinical trial. JAMA 2011;305:569-575.

12. Bundred NJ, Barnes NL, Rutgers E, Donker M. Is axillary lymph node clearance required in node-positive breast cancer? Nat Rev Clin Oncol 2015;12(1):55-61.

13. Donker M, Straver ME, van Tienhoven G, et al. Comparison of the sentinel node procedure between patients with multifocal and unifocal breast cancer in the EORTC 10981-22023 AMAROS trial: identification rate and nodal outcome. Eur J Cancer 2013;49:2093-2100.

14. Donker M, van Tienhoven G, Straver ME, et al. Radiotherapy or surgery of the axilla after a positive sentinel node in breast cancer (EORTC 10981-22023 AMAROS): a randomised, multicentre, open-label, phase 3 non-inferiority trial. Lancet Oncol 2014;15(12):1303-1310.

\section{Breast reconstruction}

1. Albornoz $C R$, Bach $P B$, Mehrara $B$ J, et al. A paradigm shift in U.S. breast reconstruction: increasing implant rates. Plast Reconstr Surg 2013;131:15-23.

2. Chang El, Liu TS, Festekjian JH, et al. Effects of radiation therapy for breast cancer based on type of free flap reconstruction. Plast Reconstr Surg 2013;131(1):1e-8e.

3. Card A, Crosby MA, Liu J, et al. Reduced incidence of breast cancer-related lymphedema following mastectomy and breast reconstruction versus mastectomy alone. Plast Reconstr Surg 2012;130(6):1169-1178.

4. Garvey PB, Villa MT, Rozanski AT, et al. The advantages of free abdominal-based flaps over implants for breast reconstruction in obese patients. Plast Reconstr Surg 2012;130(5):991-1000.

5. Seth AK, Hirsch EM, Kim JYS, et al. Long-term outcomes following fat grafting in prosthetic breast reconstruction: a comparative analysis. Plast Reconstr Surg 2012:130(5):984-990.

6. Parikh RP, Doren EL, Mooney BS, et al. Differentiating fat necrosis from recurrent malignancy in fat-grafted breasts: an imaging classification system to guide management. Plast Reconstr Surg 2012;130(4):761-772.

7. Kronowitz SJ. Current status of implant-based breast reconstruction in patients receiving postmastectomy radiation therapy. Plast Reconstr Surg 2012;130(4):513-523.

8. Kronowitz SJ. Current status of autologous tissue-based breast reconstruction in patients receiving postmastectomy radiation therapy. Plast Reconstr Surg 2012;130(2):282-292.

9. HuE, Alderman AK. Breast reconstruction. Surg Clin N Am 2007;87:453-467.

10. Granzow JW, Levine JL, Chiu ES, Allen RJ. Breast reconstruction with the deep inferior epigastric perforator flap: History and an update on current technique. JPRAS 2006;59:571-579.

11. Blondeel N, Vanderstraeten GG, Monstrey SJ, Van Landuyt K, et al. The donor site morbidity of free DIEP flaps and free TRAM flaps for breast reconstruction. Br J Plast Surg 1997;50(5):322-330.

12. Nahabedian MY, Momen B, Galdino G, et al. Breast reconstruction with the free TRAM or DIEP flap: patient selection, choice of flap, and outcome. Plast Reconstr Surg 2002;110(2):466-475.

13. Evans GRD, David CL, Loyer EM, et al. The long-term effects of internal mammary chain irradiation and its role in the vascular supply of the pedicled transverse rectus abdominis musculocutaneous flap breast reconstruction. Ann Plast Surg 1995;35:342-348.

14. Neligan PC. Preoperative imaging techniques for perforator selection in abdomen-based microsurgical breast reconstruction. Clin Plast Surg 2010;37(4):581-591.

15. Padubidri AN, Yetman R, Browne E, et al. Complications of postmastectomy breast reconstructions in smokers, ex-smokers, and nonsmokers. Plast Reconstr Surg 2001;107(2):342-349.

16. Santamaría-Linares E, Ramirez-Ugalde MT, Ochoa-Carrillo F, Fuentes Alburo A. Reconstrucción mamaria con colgajo TRAM libre. ¿Se justifica el riesgo? Cir Plast 2001;11(2):49-60.

17. Schaverien M, Douglas R, McCulley SJ. Is immediate autologous breast reconstruction with postoperative radiotherapy good practice? A systematic review of the literature. JPRAS 2013;66:1637-1651.

18. Kelley BP, Ahmed R, Kidewell KM, et al. A systematic review of morbidity associated with autologous breast reconstruction before and after exposure to radiation therapy - are current practices ideal? Ann Surg Oncol 2014;21(5):1732-1738.

19. Berbers J, van Baardwijk A, Houben R, et al. 'Reconstruction: before or after mastectomy radiotherapy?' A systematic review of the literature. Eur J Cancer 2014:50:2752-2762. 


\section{Risk-reducing mastectomy}

1. Smith RA, Cokkinides V, Brawley OW. Cancer screening in the United States, 2009: A review of current American Cancer Society guidelines and issues in cancer screening. CA Cancer J Clin 2009;59:27-41.

2. Breast cancer screening and diagnosis, 2012 NCCN Clinical Practice Guidelines in Oncology.

3. Hereditary breast and/or ovarian cancer, 2012. NCCN Clinical Practice Guidelines in Oncology.

4. Dragun AE, Pan J, Riley EC, et al. Increasing use of elective mastectomy and contralateral prophylactic surgery among breast conservation candidates: a 14-year report from a comprehensive cancer center. Am J Clin Oncol 2013;36(4):375-380

5. Chung A, Huynh K, Lawrence C, et al. Comparison of patient characteristics and outcome of contralateral prophylactic mastectomy and unilateral total mastectomy in breast cancer patients. Ann Surg Oncol 2012;19:2600-2606

\section{Adjuvant systemic therapy at operable stages}

\section{Introduction and adjuvant treatment with chemotherapy}

1. Early Breast Cancer Trialists' Collaborative Group (EBCTCG). Effects of chemotherapy and hormonal therapy for early breast cancer on recurrence and 15-year survival: an overview of the randomised trials. Lancet 2005;365:1687-1717.

2. Early Breast Cancer Trialists' Collaborative Group (EBCTCG), Peto R, Davies $\mathrm{C}$, et al. Comparisons between different polychemotherapy regimens for early breast cancer: meta-analyses of long-term outcome among 100,000 women in 123 randomised trials. Lancet 2012;379:432-44.

3. Invasive Breast Cancer 2017, NCCN Clinical Practise Guidelines in Oncology

4. Aebi S, Davidson T, Gruber G, et al. Primary breast cancer: ESMO clinical practice guidelines for diagnosis, treatment and follow-up. Ann Oncol 2011;22(suppl 6):vi12-24.

5. Goldhirsch A, Wood WC, Coates AS, et al. Strategies for subtypesdealing with the diversity of breast cancer: highlights of the St. Gallen International Expert Consensus on the Primary Therapy of Early Breast Cancer 2011. Ann Oncol 2011;22:1736-1747

6. Chavez-MacGregor M, Clarke CA, Lichtensztajn DY, Giordano SH. Delayed Initiation of Adjuvant Chemotherapy among Patients with Breast Cancer. JAMA Oncol 2016;2(3):322-329.

7. Ferguson T, Wilcken N, Vagg R, et al. Taxanes for adjuvant treatment of early breast cancer. Cochrane Database Syst Rev 2007;4:CD004421.

8. De Laurentiis M, Cancello G, D'Agostino D, et al. Taxane-based combinations as adjuvant chemotherapy of early breast cancer: a meta-analysis of randomized trials. J Clin Oncol 2008;26:44-53.

9. Assikis V, Buzdar A, Yang Y, et al. A phase III trial of sequential adjuvant chemotherapy for operable breast carcinoma: final analysis with 10-year follow-up. Cancer 2003:97(11):2716-2723.

10. Martin M, Rodriguez-Lescure A, Ruiz A, et al. Randomized phase 3 trial of fluorouracil, epirubicin, and cyclophosphamide alone or followed by paclitaxel for early breast cancer. J Natl Cancer Inst 2008;100:805-814.

11. Roche H, Fumoleau P, Spielmann M, et al. Sequential adjuvant epirubicin-based and docetaxel chemotherapy for node-positive breast cance patients: the FNCLCC PACS 01 Trial. J Clin Oncol 2006;24:5664-5671.

12. Sparano JA, Zhao F, Martino S, et al. Long-Term Follow-Up of the E1199 Phase III Trial Evaluating the Role of Taxane and Schedule in Operable Breast Cancer. J Clin Oncol 2015;33(21):2353-2360

13. Sparano JA, Wang M, Martino S, et al. Weekly paclitaxel in the adjuvant treatment of breast cancer. N Engl J Med 2008;358:1663-1671.

14. Martin M, Pienkowski T, Mackey J, et al. Adjuvant docetaxel for node-positive breast cancer. N Engl J Med 2005;352:2302-2313.

15. Jones S, Holmes FA, O'Shaughnessy J, et al. Docetaxel with cyclophosphamide is associated with an overall survival benefit compared with doxorubicin and cyclophosphamide: 7-year follow-up of US Oncology Research Trial 9735. J Clin Oncol 2009;27:1177-1183.

16. Citron ML, Berry DA, Cirrincione $C$, et al. Randomized trial of dose-dense versus conventionally scheduled and sequential versus concurrent combination chemotherapy as postoperative adjuvant treatment of node-positive primary breast cancer: first report of Intergroup Trial C9741/Cancer and Leukemia Group B Trial 9741. J Clin Oncol 2003;21:1431-1439.

\section{Adjuvant treatment with hormone therapy}

1. Cuzick J, et al. Effect of tamoxifen and radiotherapy in women with locally excised ductal carcinoma in situ: long-term results from the UK/ANZ DCIS trial. Lancet Oncol 2011;12(1):21-29.

2. Wapnir IL, et al. Long term-outcomes of invasive ipsilateral breast tumor recurrences after lumpectomy in NSABP B-27 and B-24 randomized clinical trials for DCIS. J Natl Cancer Inst 2011;103:478-488.
3. Fisher $\mathrm{B}$, et al. Tamoxifen for the prevention of breast cancer: current status of the National Surgical Adjuvant Breast and Bowel Project P-1 study. J Natl Cancer Inst 2005;97:1652-1662.

4. Margolese RG, et al. Anastrozole versus tamoxifen in postmenopausal women in ductal carcinoma in situ undergoing lumpectomy plus radiotherapy (NSAB B-35): a randomised, double-blind, phase 3 clinical trial. Lancet 2016;387:849-856.

5. Forbes J, et al. Anastrozole versus tamoxifen for the prevention of locoregional and contralateral breast cancer in postmenopausal women with locally excised ductal carcinoma in situ (IBIS-II DCIS): a double-blind, randomised controlled trial. Lancet 2016;387:866-873.

6. Pagani $O$, et al. Adjuvant Exemestane with Ovarian Suppression in Premenopausal Breast Cancer. NEJM 2014;371:107-118.

7. Francis PA, et al. Adjuvant Ovarian Supression in Premenopausa Breast Cancer. N Engl J Med 2015;372:436-446. DOI: 10.1056/NEJMoa1412379.

8. Tjan-Henjnen VC, et al. First results from the multicenter phase III DATA study comparing 3 versus 6 years of anastrozole after 2-3 years of tamoxifen in postmenopausal women with hormone receptor-positive early breast cancer. SABCS 2016:S1-03. doi: 10.1158/1538-7445.

9. Mamounsas EP, et al. A randomized, double-blinded, placebo-controlled clinical trial to evaluate extended adjuvant endocrine therapy (5 years of letrozole) in postmenopausal women with hormone-receptor positive breast cancer who have completed previous adjuvant endocrine therapy: initial results of NRG oncology/NSABP- B42. SABCS 2016:S1-05.

10. Invasive Breast Cancer. Version 12016 , NCCN Clinical Practice Guidelines in Oncology.

11. Blok EJ, et al. Optimal duration of extended letrozole treatment after 5 years of adjuvant endocrine therapy; results of the randomized phase III IDEAL trial (BOOG 2006-05). SABCS 2016:S1-04.

12. Goss PE, et al. Extending Aromatase-Inhibitor Adjuvant Therapy to 10 Years. N Engl J Med 2016;375:209-219.

\section{Adjuvant treatment with targeted therapies (trastuzumab)}

1. Zardavas D, Tryfonidis K, Goulioti T, Piccart M. Targeted adjuvant therapy in breast cancer. Exp Rev Anticancer Ther 2016;16(12):1263-1275.

2. Dahabreh IJ, Linardou $\mathrm{H}$, Siannis $\mathrm{F}$, et al. Trastuzumab in the adjuvan treatment of early-stage breast cancer: a systematic review and meta-analysis of randomized controlled trials. Oncologist 2008;13:620-630.

3. Gianni L, Dafni U, Gelber RD, et al. Treatment with trastuzumab for 1 year after adjuvant chemotherapy in patients with HER2-positive early breast cancer: a 4-year follow-up of a randomised controlled trial. Lancet Oncol 2011;12:236-244.

4. Pérez EA, Romond E, Suman VJ, et al. Trastuzumab plus adjuvant chemotherapy for human epidermal growth factor receptor 2-positive breast cancer: planned joint analysis of overall survival (OS) from NSABP B-31 and NCCTG N9831. J Clin Oncol 2014;32(33):3744-3752.

5. Lambertini M, Pondé NF, Solinas C; de Azambuja E. Adjuvant trastuzumab: a 10-year overview of its benefit. Exp Rev Anticancer Ther 2017:17(1):61-74. doi: 10.1080/14737140.2017.1264876.

6. Slamon DJ, Eiermann W, Robert NJ, et al. Ten year follow-up of the BCIRG-006 trial comparing doxorubicin plus cyclophosphamide followed by docetaxel (ACT) with doxorubicin plus cyclophosphamide followed by docetaxel and trastuzumab (ACTH) with docetaxel,carboplatin and trastuzumab $(\mathrm{TCH})$ in HER2+ early breast cancer patients. San Antonio Breast Cancer Sympoisum 2015; abstract S5-04.

7. Perez EA, Suman VJ, Davidson NE, et al. Sequential versus concurrent trastuzumab in adjuvant chemotherapy for breast cancer. J Clin Oncol 2011;29:4491-4497.

8. Jackisch C, Piccart MJ, Gelber RD, et al. HERA trial: 10 years follow up of trastuzumab after adjuvant chemotherapy in HER2 positive early breast cancer - Final analysis. San Antonio Breast Cancer Sympoisum 2015; abstract PD5-01.

9. Pivot X, Romieu G, Debled M. et al 6 months versus 12 months of adjuvant trastuzumab for patients with HER2-positive early breast cancer (PHARE): a randomised phase 3 trial. Lancet Oncol 2013; 14(8):741-748

10. Tolaney SM, Barry WT, Dang CT, et al. Adjuvant paclitaxel and trastuzumab for nodenegative, HER2-positive breast cancer. N Engl J Med. 2015;372(2):134-141.

\section{Breast cancer medical treatment-derived mid and long-term toxicity}

1. From Cancer patient to cancer survivor, IOM (Institute of Medicine) Report 2006 ISBN 0-309-09595-6.

2. Pinder MC, Duan Z, Goodwin J, et al. Congestive heart failure in older women treated with adjuvant anthracyline chemotherapy for breast cancer. J Clin Oncol 2007:25:3808-3815. 
3. Romond $E H$, Jeong $J H$, Rastogi $P$, et al. Seven-year follow-up assessment of cardiac function in NSABP B-31, a randomized trial comparing doxorubicin and cyclophosphamide followed by paclitaxel (ACP) with ACP plus trastuzumab as adjuvant therapy for patients with node-positive, human epidermal growth factor receptor 2-positive breast cancer. J Clin Oncol 2012;30(31):3792-3799.

4. Chavez-Macgregor M, Zhang N, Buchholz TA, et al. Trastuzumab-related cardiotoxicity among older patients with breast cancer. J Clin Oncol 2013;31(33):4222-4228.

5. Patt DA, Duan Z, Fang $S$, et al. Acute myeloid leukemia after adjuvant breast cancer therapy in older women: understanding the risk. J Clin Oncol 2007;25(25):3871-3876.

6. Wolff AC, Blackford AL, Visvanathan K, et al. Risk of marrow neoplasms after adjuvant breast cancer therapy: the national comprehensive cancer network experience. J Clin Oncol 2015;33(4):340-348.

7. Schneider B, Zhao F, Wang M, et al. Neuropathy is not associated with clinical outcomes in patients receiving adjuvant taxane-containing therapy for operable breast cancer. J Clin Oncol 2012;30(25):3051-3057.

8. Smith EM, Pang $\mathrm{H}$, Cirrincione $\mathrm{C}$, et al. Effect of duloxetine on pain, function and QoL among patients with painful chemotherapy-induced neuropathy. JAMA 2013;309(13):1359-1367.

9. Hershman DL, Lacchetti C, Dworkin $\mathrm{RH}$, et al. Prevention and management of chemotherapy-induced peripheral neuropathy-ASCO clinical practice guideline. J Clin Oncol 2014;32(18):1941-1967.

10. Bower JE, Bak K, Breitbart W, et al. Screening, assessment and management of fatigue in adult survivors of cancer: An ASCO practice guideline adaptation. J Clin Oncol 2014;32(17):1840-1850.

11. NCCN Clinical Practice Guidelines in Oncology. Cancer-related fatigue version2.2017- April 2017, National Comprehensive Cancer Network (https://www.nccn.org/professionals/physician_gls/pdf/fatigue.pdf.)

12. Pachman DR, Barton DL, Swetz KM, Loprinzi C. Troublesome symptoms in cancer survivors: fatigue, insomnia, neuropathy, and pain. J Clin Oncol 2012;30:30:3687-3696.

13. Azim HA, Azanbuja E, Colozza M, et al. Long-term toxic effects of adjuvant chemotherapy in breast cancer. Ann Oncol 2011;22(9):1939-1947.

14. Mann E, Smith MJ, Hellier J, et al. Cognitive behavioural treatment for women who have menopausal symptoms after breast cancer treatment (MENOS 1): a randomised controlled trial. Lancet Oncol 2012;13:309-318.

15. Azim HA, Kroman HA, Paesmans A, et al. Prognostic impact of pregnancy after breast cancer according to estrogen receptor status: A multicenter retrospective study. J Clin Oncol 2013;31(1):73-79.

\section{Adjuvant radiotherapy}

1. Kirova YM. Radiation therapy (RT) after breast-conserving surgery (BCS) in 2015- the year of radiation therapy advances. Eur J Surg Oncol 2016;42(4):437-440.

2. Shaitelman SF, Sclembach PJ, Arzu I, et al. Acute and short term toxic effects of conventionally fractionated vs hypofractionated whole breast irradiation: a randomized clinical trial. JAMA Oncol 2015;1:931-941.

3. Budach W, Bölke E, Matuschek C. Hypofractionated Radiotherapy as Adjuvant Treatment in Early Breast Cancer. A Review and Meta-Analysis of Randomized Controlled Trials. Breast Care (Basel) 2015;10:240-245.

4. Valle LF, Agarwal S, Bickel KE. Hypofractionated whole breast radiotherapy in breast conservation for early stage breast cancer: a systematic review and meta-analysis of randomized trials. Breast Cancer Res Trea 2017:162(3):409-417. doi: 10.1007/s10549-017-4118-7.

5. Polgár $\mathrm{C}$, Ott $\mathrm{O}$, Hildebrandt $\mathrm{G}$, et al. Late side effects and cosmetic results of accelerated partial breast irradiation with interstitial brachytherapy versus whole breast irradiation after breast conserving surgery for low risk invasive and in situ carcinoma of the female breast: 5 years results of a randomised, controlled, phase 3 trial. Lancet Oncol 2017;18(2):259-268.

6. Vicini $F$, Shah $\mathrm{CH}$, Tendulkar $\mathrm{R}$, et al. Accelerated partial breast irradiation an update on published level I evidence. Brachytherapy 2016;15(5):607-615.

7. Correa C, Harris EE, Leonardi MC, et al. Accelerated Partial Breast Irradiation: Executive summary for the update of an ASTRO Evidence-Based Consensus Statement. Pract Radiat Oncol 2017;7(2):73-79. doi: 10.1016/j.prro.2016.09.007.

8. Flores-Balcázar CH, Flores-Luna L, Villarreal-Garza CM, et al. Long Waiting Lists for Initiation of Adjuvant Radiation Therapy Adversely Affects Survival in Breast Cancer Patients: A thing to Consider in Overcrowded Radiation Therapy Facilities. Int J Radiat Oncol Biol Phys 2016, 96(2): E409.

9. Rodin D, Longo J, Sherertz T, et al. Mobilising Expertise and Resources to Close the Radiotherapy Gap in Cancer Care. Clin Oncol 2017:29:135-140.

10. Chan S, Rowbottom L, McDonald R, et al G.A. Does the Time of Radiotherapy Affect Treatment Outcomes? A Review of the Literature. Clin Oncol 2017;29(4):231-238. doi 10.1016/j.clon.2016.12.005.

11. Hennequin C, Barillot I, Azria D, Belkacemi $Y$, et al. Radiothérapie du cancer du sein. Cancer/Radiothérapie 2016;20:S139-S146.

12. Speers C, Pierce LJ. Postoperative Radiotherapy after Breast-Conserving Surgery for Early-Stage Breast Cancer: A review. JAMA Onco 2016:2(8):1075-1082.
13. Ponzone R, Ruatta $\mathrm{F}$, Gatti $\mathrm{M}$, et al. Omission of axillary dissection after a positive sentinel lymph-node: Implications in the multidisciplinary treatment of operable breast cancer. Cancer Treat Rev 2016;48:1-7.

14. Swanick CW, Smith BD. Indications for adjuvant radiation therapy in breast cancer: a review of the evidence and recommendations for clinical practice. Chin Clin Oncol 2016;5(3):38-51.

15. Budach W, Bölke E, Kammers K, et al. Adjuvant radiation therapy of regional lymph nodes in breast cancer - a meta-analysis of randomized trials - an update. Radiat Oncol 2015;10:258.

16. Wong JS, Warren LEG, Bellon JR. Management of the Regional Lymph Nodes in Early-Stage Breast Cancer. Semin Radiat Oncol 2016:26:37-44.

17. Whelan TJ, Olivotto IA, Parulekar WR, et al. MA20 Study Investigators. Regional nodal irradiation in early-stage breast cancer. $\mathrm{N}$ Engl J Med 2015:373(4):307-316.

18. Verma V, Vicini F, Tendulkar RD, et al. Role of Internal Mammary Node Radiation as a Part of Modern Breast Cancer Radiation Therapy: A Systematic Review. Int J Radiat Biol Phys 2016;95(2):617-631:

19. Poortmans PM Collete S, Kirove $C$, et al. Internal Mammary and Medial Supraclavicular irradiation in Breast Cancer. N Engl J Med 2015;373:317-327.

20. Bazan JG, White JR. The role of Postmastectomy Radiation Therapy in Patients with Breast Cancer Responding to Neoadjuvant Chemotherapy. Semin Radiat Oncol 2016;26:51-58.

21. Recht A, Comen EA, Fine RE, et al. Postmastectomy Radiotherapy: An American Society of Clinical Oncology, American Society for Radiation Oncology, and Society of Surgical Oncology Focused Guideline Update. Ann Surg Oncol 2017;24:38-51.

22. Cecchini MJ, Yu E, Potvin K, et al. Concurrent or Sequential Hormonal and Radiation Therapy in Breast Cancer: A Literature Review. Cureus 2015;7(10):e364.

23. Li YF, Chang L. Radiotherapy concurrent versus sequential with endocrine therapy in breast cancer: A meta-analysis. Breast 2016;27:93-98.

XIV. Neoadjuvant treatment of stage II and III breast cancer, including locally advanced disease

1. Loibl S, Denkert C, von Minckwitz. Neoadjuvant treatment of brast cancer - Clinical and research perspective. Breast 2015;24(Suppl 2):S73-S77.

2. Von Minckwitz G, Unth M, Blohmer JU, et al. Definition and impact of pathologic complete response on prognosis after neoadjuvant chemotherapy in various intrinsic breast cancer subtypes. J Clin Oncol 2012;30:1796-1804.

3. Donker M, Straver ME, Rutgers EJ, et al. Radioguided occult lesion localisation (ROLL) in breast-conserving surgery after neoadjuvant chemotherapy. EJSO 2012;38(12):1218-1224.

4. Kaufmann M, von Minckwitz G, Mamounas EP, et al. Recommendations from an international consensus conference on the current status and future of neoadjuvant systemic therapy in breast cancer. Ann Surg Oncol 2012;19:1508-1516.

5. Von Minckwitz G, Untch M, Nuesch E, et al. Impact of treatment characteristics on response of different breast cancer phenotypes: pooled analysis of the German neoadjuvant chemotherapy trials. Breast Cancer Res Treat 2011;125:145-156.

6. Sikov WM, Berry DA, Perou C, et al. Impact of the addition of carboplatin and or bevacizumab to neoadjuvant once-per-week paclitaxel followed by dose-dense doxorrubicin and cyclophosphamide on pathologic complete response rates in stage II to III triple negative breast cancer: CALBG 4063 (Alliance). J Clin Oncol 2015:33:13-21.

7. Von Minckwitz G, Schneeweiss A, Loibl S, et al. Neoadjuvant carboplatin with triple negative and HER2-positive early breast cancer (GeparSixto GBG66): A randomised phase 2 trial. Lancet Oncol 2014;15:747-756.

8. Earl HM, Valler Al, Hiller L, et al. Effects of the addition of gemcitabine and paclitaxel-first sequencing in neoadjuvant sequential epirubicin, cyclophosphamide and paclitaxel for women with high risk early breast cancer (Neo-tAnGo): an open label 2x2 factorial randomised phase 3 trial. Lancet Oncol 2014;15:201-212.

9. Steger GG, Greil R, Lang A, et al. Epirubicin and docetaxel with or without capecitabine as neoadjuvant treatment for early breast cancer: final results of a randomized phase III study (ABCSG-24). Ann Oncol 2014;25:366-371.

10. Untch M, Jackish $C$, Schneeweiss $A$, et al. Nab-paclitaxel versus solvent-based paclitaxel in neoadjuvant chemotherapy for early breast cancer (GeparSepto-GBG 69) a randomised, phase 3 trial. Lancet Oncol 2016;17:345-356.

11. ETNA (Evaluating Treatment with Neoadjuvant Abraxane) randomized phase III study comparing neoadjuvant nab-paclitaxel (nab-P) versus paclitaxel $(P)$ both followed anthracycline regimens in women with HER2-negative high risk breast cancer: A MICHELANGELO study. J Clin Oncol 2016;34(suppl; abstr 502).

12. Gianni L, Eiermann W, Semiglazov V, et al. Neoadjuvant and adjuvant trastuzumab in patients with HER2-positive locally advanced breast cancer (NOAH): follow-up of a randomised controlled superiority trial with a parallel HER2-negative cohort. Lancet Oncol 2014;15:640-647. 
13. Untch M, Fasching PA, Konecny GE, et al. Pathologic complete response after neoadjuvant chemotherapy plus trastuzumab predicts favorable survival in human epidermal growth factor receptor 2-everexpressing breast cancer: results from the TECHNO trial of the AGO and GBG study groups. J Clin Oncol 2011;29:3351-3357.

14. Buzdar AU, Ibrahim NK, Francis D, et al. Significantly higher pathologic complete remission rate after neoadjuvant therapy with trastuzumab, paclitaxel and epirubicin chemotherapy: results of a randomised tria in human epidermal growth factor receptor 2-positive operable breast cancer. J Clin Oncol 2005;23:3676-3685.

15. Buzdar AU, Suman VJ, Meric-Bernstam F, et al. Fluorouracil, epirubicin, and cyclophosphamide (FEC-75) followed by paclitaxel plus trastuzumab versus pacitaxel plus trastuzumab followed by FEC-75 plus trastuzumab as neoadjuvant treatment for patients with HER2-positive breast cancer (Z1041): a randomised, controlled, phase 3 trial. Lancet Oncol 2013;14:1317-1325.

16. Robidoux A, Tang G, Rastogi $P$, et al. Lapatinib as component of neoadjuvant therapy for HER2-positive operable breast cancer (NSABP protocol B-41): an open-lebel, randomised trial. Lancet Oncol 2013;14:1183-1192.

17. Hanusch C, Schneeweiss A, Loibl S, et al. Dual blockade with AFatinib and trastuzumab as NEoadjuvant treatment for patients with locally advanced of operable breast cancer receiving taxane-anthracycline containing chemotherapy-DAFNE (GBG-70). Clin Cancer Res 2015;21:2924-2931.

18. Hurvitz SA, Martin M, Symmans WF, et al. Pathologic complete response (pCR) rates after neoadjuvant trastuzumab emtansine (TDM-1 [K]) + pertuzumab $(\mathrm{P})$ vs docetaxel + carboplatin + trastuzumab + P (TCHP) tratment in patients with HER2-positive (HER2+) early breast cance (EBC) (KRISTINE). J Clin Oncol 2016;34(15 suppl):500-500.

19. Gianni L, Pienkowski T, Im YH, et al. 5-year analysis of neoadjuvant pertuzumab and trastuzumab in patients with locally advanced, inflammatory, or early-stage HER2-positive breast cancer (NeoSphere): a multicentre, open-label, phase 2 randomised trial. Lancet Oncol 2016;17:791-800.

20. Schneeweiss A, Chia S, Hickish T, et al. Pertuzumab plus trastuzumab in combination with standard neoadjuvant anthracycline-containing and anthracycline-free chemotherapy regimens in patients with HER2-positive early breast cancer: a randomized phase II cardiac safety study (TRYPHAENA). Ann Oncol 2013;24:2278-2284.

21. Bear DH, Tang $G$, Rastogi $P$, et al. Bevacizumab added to neoadjuvant chemotherapy for breast cancer. N Engl J Med 2012;366:310-320.

22. Cataliotti L, Buzdar AU, Noguchi S, et al. Comparison of anastrazol versus tamoxifen as preoperative therapy in postmenopausal women with hormone-receptor positive breast cancer: the pre-operative "Arimidex" compared to tamoxifen (PROACT) trial. Cancer 2006;106:2095-2103.

23. Eiermann W, Paepke S, Appfelstaedth J, et al. Preoperative treatmen of postmenopausal breast cancer patients with letrozole: a randomized double-blind multicenter study. Ann Oncol 2001;12:1527-1532.

24. Allevi G, Strina C, Andreis D, et al. Increased pathological complete response rate after a long-term neoadjuvant letrozole treatment in postmenopausal oestrogen and/or progesterone receptor-positive breast cancer. Br. J Cancer 2013;108:1587-1592.

\section{Post-neoadjuvancy treatment}

1. Nakahara $H$, Yasuda $Y$, Machida $E$, et al. MR and US imaging for breast cancer patients who underwent conservation surgery after neoadjuvan chemotherapy: comparison of triple negative breast cancer and other intrinsic subtypes. Breast Cancer 2011;18:152-160.

2. Donker M, Straver ME, Rutgers EJ, et al. Radioguided occult lesion localization (ROLL) in breast-conserving surgery after neoadjuvant chemotherapy. Eur J Surg Oncol 2012;38:1218-1224.

3. Straver ME, Rutgers EJ, Rodenhius $S$, et al. The relevance of breast cancer subtypes in the outcome of neoadjuvant chemotherapy. Ann Surg Oncol 2010;17:2411-2418.

4. Semiglazov V, Eiermann W, Zambetti M, et al. Surgery following neoadjuvant therapy in patients with HER2 positive locally advanced or inflammatory breast cancer participating in the neoadjuvant herceptin (NOAH) study. Eur J Surg Oncol 2011;37:856-863.

5. Rouzier R, Mathieu MC, Sideris L, et al. Breast-conserving surgery after neoadjuvant anthracycline based chemotherapy for large breast tumors. Cancer 2004;101:918-925.

6. Espinosa-Bravo M, Sao-Avilés A, Esgueva A, et al. Breast conservative surgery after neoadjuvant chemotherapy in breast cancer patients: comparison of two tumor localization methods. Eur J Surg Oncol 2011;37:1038-1043.

7. Straver ME, Rutgers EJ, Oldenburg HS, et al. Accurate axillary lymph node dissection is feasible after neoadjuvant chemotherapy. Am J Surg 2009;198:46-50.

8. Hunt KK, Yi M, Mittendorf EA, et al. Sentinel lymph node surgery after neoadjuvant chemotherapy is accurate and reduces the need of axillary dissection in breast cancer patients. Ann Surg 2009;250:558-566.

9. Decker MR, Breenblatt DY, Havlena J, et al. Impact of neoadjuvant chemotherapy on wound complications after breast surgery. Surgery 2012;152:382-388.

\section{Radiotherapy aspects}

1. Swanick CW, Smith BD. Indications for adjuvant radiation therapy in breast cancer: a review of the evidence a recommendations for clinical practice. Chin Clin Oncol 2016;5(3):3-13.

2. Recht A, Comen E, Fine RE, et al. Postmastectomy radiotherapy: an American Society of Clinical Oncology, American Society for Radiation Oncology and Society of Surgical Oncology focused guideline update. J Clin Oncol 2016;34(36):4431-4442. doi: 10.1200/JCO.2016.69.1188.

3. Bazan JG, White JR. The role of postmastecomy radiation therapy in patients with breast cancer responding to chemotherapy. Sem Radiat Oncol 2016;26:51-58. doi: 10.1016/j.semradonc.2015.08.001.

4. Budach W, Matuschek C. DEGRO practical guidelines for radiotherapy of breast cancer $\mathrm{V}$. Therapy for locally advanced and inflammatory breast cancer, as well as local therapy in cases with synchronous distant metastases. Strahlenther Onkol 2015;191:623-33. doi: 10.1007/s00066-015-0843-1.

5. Cardoso F, Costa A. 3rd ESO-ESMO international consensus guidelines for Advanced Breast Cancer (ABC3). Breast 2017;31:244-259. doi: 10.1016/j.breast.2016.10.001

\section{Radiotherapy-induced toxicity}

1. Marks LB. Use of normal tissue complication probability models in the clinic. Int J Radiat Oncol Biol Phys 2010;76(3 Suppl):S10-S19.

2. Rudra S, Al-Hallaq HA, Feng C, et al. Effect of RTOG breast/chest wall guidelines on dose-volume histogram parameters*. J Appl Clin Med Phys 2014,15(2):4547.

3. Friese CR, Harrison JM, Janz NK, et al. Treatment associated toxicities reported by patients with early stage invasive breast cancer. Cancer 2017;123(11):1925-1934. doi: 10.1002/cncr.30547.

4. Meattini I, Guenzi M, Fozza A, et al. Overview on cardiac, pulmonary and cutaneous toxicity in patients treated with adjuvant radiotherapy for breast cancer. Breast Cancer 2017;24(1):52-62. doi: 10.1007/s12282016-0694-3

5. Bazan J, DiConstanzo D, Kuhn K et al. Likelihood of unacceptable normal tissue doses in breast cancer patients undergoing regional nodal irradiation in routine clinical practice. Pract Radiat Oncol 2017;7(3):154-160.

6. Yeboa DN, Evans SB. Contemporary Breast Radiotherapy and Cardiac Toxicity. Semin Radiat Oncol 2016;26:71-78.

7. Jacob S, Pathak A, Franck D, et al. Early detection and prediction of cardiotoxicity after radiation therapy for breast cancer: the BACCARAT prospective cohort study. Radiat Oncol 2016;11:54. Doi: 10.1186/s13014016-0627-5.

8. Overgaard J, Grantzau T. Risk of second non-breast cancer among patients treated with and without postoperative radiotherapy for primary breast cancer: A systematic review and meta-analysis of population-based studies including 522,739 patients. Radiother Oncol 2016;121:402-413.

9. Bazire L, De Rycke Y, Asselain B, Fourquet A, Kirova YM. Risks of second malignancies after breast cancer treatment: Long-term results. Cancer Radiother 2017;21(1):10-15. doi: 10.1016/j.canrad.2016.07.101.

\section{Metastatic breast cancer treatment}

\section{Introduction}

1. Cardoso F, Costa A, Senkus E, Aapro M, et al. 3rd ESO-ESMO International Consensus Guidelines for Advanced Breast Cancer (ABC 3) Ann Oncol (2016) doi: 10.1093/annonc/mdw544 and The Breast 2016, doi: dx.doi.org/10.1016/j.breast.2016.10.001.

2. Chia SK, Speers CH, D'yachkova Y, et al. The impact of new chemotherapeutic and hormone agents on survival in a population-based cohort of women with metastatic breast cancer. Cancer 2007;110:973-979.

3. Giordano SH, Buzdar AU, Smith TL, et al. Is breast cancer survival improving? Cancer 2004;100:44-52.

4. Partridge A, Bryan R, Carey L, Come S, et al. chemotherapy and targeted therapy for woman with human epidermal growth factor receptor 2- negative (or unknow) adavanced breast cancer: American Society of Clinical Oncology Clinical Practice Guideline. J Clin Oncol 2014;32(29):3307-2229.

5. Amir E, Miller N, Geddie W, et al. Do the results of metastatic breast cancer biopsies affect patient survival outcomes? Results from a large prospective trial. Cancer Res 2010; abstract PD10-05.

6. Macfarlane, Seal M, Speers C, Masoudi H, Aparicio S et al. Molecular alterations between the primary breast cancer and subsequent locoregional/metastatic tumor. Oncologist 2012;17:172-178.

7. Van Poznak $\mathrm{C}$, Somerfield $\mathrm{M}$, Bast $\mathrm{R}$, et al. Use of biomarkers to guide decisions on systemic therapy for women with metastatic breast cancer: American Society of Clinical Oncology clinical practice guidelines. J Clin Oncol 2015;33(24):2695-2704. 
A. Metastatic breast cancer with positive hormone receptors and negative HER-2 neu

1. Osborne CK. Tamoxifen in the treatment of breast cancer. N Engl J Med 1998:339:1609-1618.

2. Boccardo F, Rubagotti A, Perrota A, et al. Ovarian ablation versus goserelin with or without tamoxifen in pre-perimenopausal patients with advanced breast cancer: results of multicentric Italian study. Ann Oncol 1994:5:337-342.

3. Klijn J, Blamey RW, Boccardo F, et al. Combined tamoxifen and luteinizing hormone releasing hormone (LHRH) agonist alone in premenopausal advanced breast cancer: a meta-analysis of four randomized trials. $J$ Clin Oncol 2001:19:343-353.

4. Finn RS, Martin M, Rugo HS, et al. Palbociclib and letrozole in advanced breast cancer. N Engl J Med 2016;375:1925-1936.

5. Mauri D, Pavlidis N, Polyzos NP, loannidis JP. Survival with aromatase inhibitors and activators versus standard hormonal therapy in advanced breast cancer: metaanalysis. J Natl Cancer Inst 2006;98:1285-1291.

6. Robertson J, Bondarenko I, Trishkina E, et al. Fulvestrant $500 \mathrm{mg}$ versus anastrozole $1 \mathrm{mg}$ for hormone receptor-positive advanced breast cancer (FALCON): an international, randomised, double-blind phase 3 trial. Lancet 2016;388:2997-3005.

7. Lonning PE, Bajetta $E$, Murray $R$, et al. Activity of exemestane in metastatic breast cancer after failure of nonsteroidal aromatase inhibitors: a phase II trial. J Clin Oncol 2000;18:2234-2244.

8. Chia S, Gradishar W, Mauriac L, et al. Double-blind, randomized placebo controlled trial of fulvestrant compared with exemestane after prior nonsteroidal aromatase inhibitor therapy in postmenopausal women with hormone receptor-positive, advanced breast cancer: results from EFECT. J Clin Oncol 2008;26:1664-1670.

9. Di Leo A, Jerusalem G, Petruzelka L, et al. Final analysis of overall survival forthe phase III CONFIRM trial: fulvestrant $500 \mathrm{mg}$ versus 250 mg. Cancer Res 2012;72:S1-4.

10. Piccart M, Baselga J, Noguchi S, et al. Final progression-free survival analysis of BOLERO-2: a phase III trial of everolimus for postmenopausal women with advanced breast cancer [SABCS abstract P6-04-02]. Cancer Res. 2012;72(suppl 3).

11. Baselga J, Campone M, Piccart M, et al. Everolimus in postmenopausal hormonereceptor-positive advanced breast cancer. $\mathrm{N}$ Engl J Med 2012;366:520-529.

12. Cristofanilli M, Turner NC, Bondarenko I, et al. Fulvestrant plus palbociclib versus fulvestrant plus placebo for treatment of hormone-receptor-positive, HER2-negative metastatic breast cancer that progressed on previous endocrine therapy (PALOMA-3): final analysis of the multicentre, double-blind, phase 3 randomised controlled trial. Lancet Oncol 2016;17(4):425-439.

13. Howell A, Dodwell DJ, Anderson H, Redford J. Response after withdrawal of tamoxifen and progestogens in advanced breast cancer. Ann Oncol 1992;3:611-617.

B. Hormone receptor-positive, HER-2 neu-positive metastatic breast cancer

C. Triple-negative breast cancer or with positive hormone receptors, negative HER-2 neu not candidate to hormone therapy

D. HER-2 neu-positive metastatic breast cancer

1. Clinical Practice Guidelines in Oncology (NCCN guidelines). Breast Cancer version 1.2017. Disponible en: www.nccn.org. Acceso el 01/02/2017.

2. Cardoso F, Costa A, Senkus E, Aapro M, et al. 3rd ESO-ESMO International Consensus Guidelines for Advanced Breast Cancer (ABC 3). Ann Oncol 2017;28:16-33.

3. Johnston S, Pergram M, Press M, et al. Lapatinib combined with letrozole vs. letrozole alone for front line postmenopausal hormone receptor positive $(\mathrm{HR}+)$ metastatic breast cancer (MBC). J Clin Onco 2009;27:5538-5546.

4. Kaufman B, Mackey JR, Clemens M, et al. Trastuzumab Plus Anastrozole Versus Anastrozole Alone for the Treatment of Postmenopausal Women With Human Epidermal Growth Factor Receptor 2-Positive, Hormone Receptor-Positive Metastatic Breast Cancer: Results From the Randomized Phase III TAnDEM Study J Clin Oncol 2009;27:5529-5537.

5. Partridge Ann, Rumble B, Carey L, Come S, et al. Chemotherapy and targeted therapy for women with human epidermal growth factor receptor 2-negative (or unknown) advanced breast cancer : American Society of Clinical Oncology clinical practice guideline J Clin Oncol 2014;32:1-23.

6. Carrick S, Parker S, Wilcken N, et al. Single agent versus combination chemotherapy for metastatic breast cancer. Cochrane Database Syst Rev 2005;2:CD003372.

7. Conte PF, Guarneri V, Bruzzi P, et al. Concomitant versus sequential administration of epirubicin and paclitaxel as first-line therapy in metastatic breast carcinoma: results for the Gruppo Oncologico Nord Ovest randomized trial. Cancer 2004;101:704-12.
8. O'Shaughnessy J, Miles D, Vukelja S, et al. Superior survival with capecitabine plus docetaxel combination therapy in anthracycline-pretreated patients with advanced breast cancer: phase III trial results. J Clin Oncol 2002:12:2812-23.

9. Albain KS, Nag SM, Calderillo-Ruiz G, et al. Gemcitabine plus paclitaxel versus paclitaxel monotherapy in patients with metastatic breast cancer and prior anthracycline treatment. J Clin Oncol 2008:26:3950-3957.

10. Blum JL, Dees EC, Chacko A, et al. Phase II trial of capecitabine and weekly paclitaxel as first-line therapy for metastatic breast cancer. J Clin Oncol 2006;24:4384-4390.

11. Chan S, Romieu G, Huober J, et al. Phase III study of gemcitabine plus docetaxel compared with capecitabine plus docetaxel for anthracycline-pretreated patients with metastatic breast cancer. J Clin Oncol 2009;27:1753-1760.

12. Soto $\mathrm{C}$, Torrecillas $\mathrm{L}$, Reyes $\mathrm{S}$, et al. Capecitabine $(\mathrm{X})$ and taxanes in patients with anthracycline-pretreated metastatic breast cancer: sequential vs. combined therapy results from a MOSG randomized phase III trial. J Clin Oncol 2006;24:570.

13. Fumoleau P, Largillier R, Clippe C, et al. Multicentre, phase II study evaluating capecitabine monotherapy in patients with anthracycline- and taxane-pretreated metastatic breast cancer. Eur $\mathrm{J}$ Cancer 2004; $40: 536-542$

14. Seidman AD, Berry D, Cirrincione C, et al. Randomized phase III trial of weekly compared with every-3-weeks paclitaxel for metastatic breast cancer, with trastuzumab for all HER-2 overexpressors and random assignment to trastuzumab or not in HER-2 nonoverexpressors: final results of Cancer and Leukemia Group B protocol 9840. J Clin Oncol 2008;26:1642-1649.

15. Mauri D, Kamposioras K, Tsali L, et al. Overall survival benefit for weekly vs. three-weekly taxanes regimens in advanced breast cancer: $A$ meta-analysis. Cancer Treat Rev 2010;36:69.

16. Kaufman PA, Awada A, Twelves $C$, et al. A phase III, open-label, randomized, multicenter study of eribulin mesylate versus capecitabine in patients with locally advanced or metastatic breast cancer previously treated with anthracyclines and taxanes. Cancer Res 2012;72:S6-6.

17. Cortes J, O'Shaughnessy J, Loesch D, et al. Eribulin monotherapy versus treatment of physician's choice in patients with metastatic breast cancer (EMBRACE): a phase 3 open-label randomised study. Lancet 2011;377:914-923

18. Kaufman $\mathrm{P}$, Awada $\mathrm{A}$, Twelves $\mathrm{C}$, et al, A phase III, open label randomized multicenter study of eribulin mesylate versus capecitabine in patients with locally advanced or metastatic breast cancer previously treated with anthracyclines and taxanes. JCO 2015;52:4892.

19. Sparano JA, Vrdoljak E, Rixe O, Xu B, Manikhas A, Medina C, et al. Randomized phase III trial of ixabepilone plus capecitabine versus capecitabine in patients with metastatic breast cancer previously treated with an anthracycline and a taxane. J Clin Oncol 2010;28(20):3256-63

20. Fan $Y, X u B H, Y u a n P$, et al. Docetaxel-cisplatino migth be superior to docetaxel-capecitabine in the first-line treatment of metastatic triple-negative breast cancer. Ann Oncol 2013;24(5)1219-1225

21. Carrick S, Ghersi D, Wilcken N, Simes J. Platinum containing regimens for metastatic breast cancer. Cochrane Database Syst Rev 2004.

22. Tutt A, Ellis P, Kilbum L, et al. TNT: a randomized phase III trial of carboplatin compared to docetaxel for patients with metastatic or recurren locally advanced triple-negative or BRCA1/2 breast cancer. Cancer Res 2015;75:9.

23. Miller K, Wang M, Gralow J, et al. Paclitaxel plus bevacizumab versus paclitaxel alone for metastatic breast cancer. $N$ Engl J Med 2007;357:2666-76.

24. Miles D, Chan A, Dirix L, et al. Phase III study of bevacizumab plus docetaxel compared with placebo plus docetaxel for the first-line treatment of human epidermal growth factor receptor 2-negative metastatic breast cancer. J Clin Oncol 2010;28:3239-3247.

25. Robert NJ, Diéras V, Glaspy J, et al. RIBBON-1: randomized, double-blind, placebo-controlled, phase III trial of chemotherapy with or without bevacizumab for first-line treatment of human epidermal growth factor receptor 2-negative, locally recurrent or metastatic breast cancer. $\mathrm{J}$ Clin Oncol 2011;29:1252-1260.

26. O'Shaughnessy J, Miles D, Gray R, et al. A meta-analysis of overall survival data from three randomized trials of bevacizumab (BV) and first-line chemotherapy as treatment for patients with metastatic breast cancer (MBC). Asco Meeting Abstracts 2010:1005.

27. Miles DW, Diéras $\mathrm{V}$, Cortes $\mathrm{J}$, et al First-line bevacizumab in combination with chemotherapy for HER-2 negative metastatic breast cancer: pooled and subgroup analysis of data from 2447 patients. Ann Oncol 2013:24:2773-2780.

28. Gennari A, Sormani M, Bruzi $P$, et al. A meta-analysis of chemotherapy duration in metastatic breast cancer. Asco Meeting Abstracts 2008:1067.

29. Gennari A. Stocker M, Puntoni M, et al. Duration of chemotherapy for metastatic breast cancer: a systematic review and meta-analysis of randomized clinical trials. J Clin Oncol 2011;29:2144-2149. 
30. Swain SM, Kim SB, Cortés J, Ro J, Semiglazov V, Campone M Ciruelos E, Ferrero JM, Schneeweiss A, Knott A, Clark E, Ross G, Benyunes MC, Baselga J. Pertuzumab, trastuzumab, and docetaxel for HER2-positive metastatic breast cancer (CLEOPATRA study): overaII survival results from a randomised, double-blind, placebo-controlled, phase 3 study. Lancet Oncol 2013;14(6):461-471.

31. Baselga J, Cortés J, Kim SB, et al. Pertuzumab plus trastuzumab plus docetaxel for metastatic breast cancer. N Engl J Med 2012;366:109-119.

32. Marty M, Cognetti F, Maraninchi D, et al. Randomized phase II trial of the efficacy and safety of trastuzumab combined with docetaxel in patients with human epidermal growth factor receptor 2-positive metastatic breast cancer administered as first-line treatment: the M77001 study group. $J$ Clin Oncol 2005;23:4265-4274.

33. Andersson M, Lidbrink E, Bjerre K, et al. Phase III randomized study comparing docetaxel plus trastuzumab with vinorelbine plus trastuzumab as first-line therapy of metastatic or locally advanced human epiderma growth factor receptor 2-positive breast cancer. The HERNATA Study. J Clin Oncol 2011;29:264-271.

34. Verma S, Miles D, Gianni L, et al. Trastuzumab emtansine for HER2-positive advanced breast cancer. N Engl J Med 2012;367(19):1783-1791.

35. Geyer CE, Forster J, Lindquist D, et al. Lapatinib plus capecitabine for HER2-positive advanced breast cancer. N Engl J Med 2006;355:2733-2743

36. Blackwell KL, Burstein HJ, Storniolo AM, et al. Randomized study of Lapatinib alone or in combination with trastuzumab in women with $\mathrm{Er}$ bB2-positive, trastuzumab-refractory metastatic breast cancer. J Clin Oncol 2010;28:1124-30.

37. von Minckwitz G, du Bois A, Schmidt M, et al. Trastuzumab beyond progression in human epidermal growth factor receptor 2-positive advanced breast cancer: a German breast group 26/breast international group 03-05 study. J Clin Oncol 2009;27:1999-2006.

\section{The role of surgery in metastatic disease}

1. Pockaj BA, Wasif N, Dueck AC, Wigle DA, Boughey JC, Degnim AC, et al. Metastasectomy and surgical resection of the primary tumor in patients with stage IV breast cancer. Time for a second look? Ann Surg Oncol 2010;17:2419-2426.

2. Kobayashi T, Ichiba T, Sakuyama T, et al. Possible clinical cure of metastatic breast cancer: lessons from 30-year experience with oligometastatic breast cancer patients and literature review. Breast Cancer 2012; 19:218-237.

3. Simmonds PC, Primrose JN, Colquitt JL, Garden OJ, Poston GJ, Rees M. Surgical resection of hepatic metastases from colorectal cancer: a systematic review of published studies. Br J Cancer 2006;94:982-999.

4. Zegarac M, Nikolic S, Gavrilovic D et al. Prognostic factors for longer disease free survival and overall survival after surgical resection of isolated liver metastasis from breast cancer. J Buon 2013;18:859-865.

5. Bacalbasa N, Dima SO, Purtan-Purnichescu R, Herlea V, Popescu I. Role of surgical treatment in breast cancer liver metastases: a single center experience. Anticancer Res 2014;34:5563-5568.

6. Abbot DE, Brouquet A, Mittendorf EA, et al. Resection of liver metastases from breast cancer: estrogen receptor status and response to chemotherapy before metastasectomy define outcome. Surgery 2012;151:710-716.

7. Yoshimoto M, Tada K, Nishimura S, Makita M, Iwase T, Kasumi F, et al Favourable long-term results after surgical removal of lung metastases of breast cancer. Breast Cancer Res Treat 2008;110:485-491.

8. Suryanarayana Deo SV, Jha D. Role of loco-regional surgery in metastatic breast cancer. J Cancer Res Ther 2013:9:181-186.

9. Van Geel AN, Wouters MW, van der Pol C, Schmitz PI, Lans T. Chest wall resection for internal mammary lymph node metastases of breast cancer. Breast 2009;18:94-9.

10. Babiera GV, Rao R, Feng L, Meric-Bernstam F, Kuerer HM, Singeltary SE, et al. Effect of primary tumor extirpation in breast cancer patients who present with stage IV disease and an intact primary tumor. Ann Surg Oncol 2006:13:776-782

11. Medina-Franco H, Suarez-Bobadilla YL. Role of surgery in metastatic breast cancer. Rev Invest Clin 2012;64:81-8.

12. Badwe R, Hawaldar R, Khare A, et al. Role of local-regional treatment in metastatic breast cancer at presentation: A randomized clinical trial. Presented at: 2008 Breast Cancer Symposium, Washington, D.C., USA, 5-7 September 2008.

13. Atilla S, Serdar O, Sheryl KF, Bahadir GM. Randomized trial comparing locoregional resection of primary tumor with no surgery in stage IV breast cancer at presentation. Breast J 2009;15:399-403.

14. Khan SA. Early surgery or standard palliative therapy in treating patients with stage IV breast cancer. National Institute of Cancer website. November 2010. www.cancer.gov/clinicaltrials.

15. Nguyen DH, Truong PT. A debate on locoregional treatment of the primary tumor in patients presenting with stage IV breast cancer. Expert Rev Anticancer Ther 2011;11:1913-1922.

16. Bergenfeldt $M$, Jensen BV, Skjoldbye $B$ et al. Liver resection and local ablation of breast cancer liver metastases - A systematic review. Eur J Surg Oncol 2011;37:549-557.

\section{The role of radiotherapy in metastatic disease}

1. Mauro GP, de Andrade Carvalho H, Stuart SR, et al. Effects of locoregional radiotherapy in patients with metastatic breast cancer. Breast 2016:28:73-78.

2. Badwe R, Hawaldar R, Nair N, et al. Locoregional treatment versus no treatment of the primary tumour in metastatic breast cancer: an open label randomised controlled trial. Lancet Oncol 2015;16:1380-1388.

3. Truong PT. Local treatment of the primary tumour inpatients presenting with stage IV breast cancer: a first, and what's up ahead. Int J Radiat Oncol Biol Phys 2017:97:443-444.

4. Qian Y, Dudley S, Durkee BY, et al. Fractionation of palliative radiotherapy in metastatic breast cancer: Selection and survival J Clin Oncol 33, no. 29 suppl (October 2015) 201-201

5. Lutz S, Balboni T, Jones J, et al. Palliative radiation therapy for bone metastases: Update of an ASTRO Evidence - Base Guideline. Pract Radiat Oncol 2017:7(1):4-12.

6. Halasz LM, Uno H, Hughes M, et al. Comparative effectiveness of stereotactic radiosurgery versus whole-brain radiation therapy for patients with brain metastases from breast or non-small cell lung cancer. Cancer 2016;122(13):2091-100.

7. Sahgal A, Aoyama H, Kocher M, et al. Phase 3 trials of stereotactic radiosurgery with or without whole-brain radiation therapy for 1 to 4 brain metastases: individual patient data meta-analysis. Int J Radiat Oncol 2015:91(4):710-717.

8. Comito T, Clerici E, Tozzi A, D'Agostino G. Liver metastases and SBRT: A new paradigm? Rep Pract Oncol Radiother 2015;20(6):464-471.

9. Gaya A, Mahadevan A, editors. Stereotactic Body Radiotherapy. London: Springer London, 2015. Available from: http://link.springer. com/10.1007/978-0-85729-597-2.

10. Ricco A, Davis J, Rate W, et al. Lung metastases treated with stereotactic body radiotherapy: the RSSearch $\circledast$ patient Registry's experience. Radiat Oncol 2017;12:35.

11. Gaya A, Mahadevan A, editors. Stereotactic Body Radiotherapy. London: Springer London, 2015. Available from: http://link.springer. $\mathrm{com} / 10.1007 / 978-0-85729-597-2$.

\section{Bisphosphonates and receptor-activator NF-KB ligand (RANKL) inhibitors} in bone metastases, adjuvancy and with aromatase inhibitors

1. Angelucci A, Alesse E. Molecular Pathology of Cancer. Metastasis: Suggestions for Future Therapy. In: Biotargets of Cancer in Current Clinical Practice, Springer, 2012:469-515.

2. Kremer R, Gagnon B, Meguerditchian AN, et al. Effect of oral bisphosphonates for osteoporosis on development of skeletal metastases in women with breast cancer: results from a pharmaco-epidemiological study. J Natl Cancer Inst 2014;106(11).

3. Hadji P, Aapro MS, Body JJ, et al. Management of aromatase inhibitor-associated bone loss in postmenopausal women with breast cancer: practical guidance for prevention and treatment. Ann Oncol 2011;22(12):2546-2555.

4. Wong MH, Stockler MR, Pavlakis N. Bisphosphonates and other bone agents for breast cancer. Cochrane Database Syst Rev 2012 Feb $15 ; 2$.

5. Lluch A, Cueva J, Ruiz-Borrego M, Ponce J, Pérez-Fidalgo JA. Zoledronic acid in the treatment of metastatic breast cancer. Anticancer Drugs 2014;25(1):1-7.

6. Stopeck AT, Lipton A, Body JJ, et al. Denosumab compared with zoledronic acid for the treatment of bone metastases in patients with advanced breast cancer: a randomised, double-blind study. J Clin Oncol 2010;28:5132-5139.

7. Barrett-Lee P, Casbard A, Abraham J, et al. Oral ibandronic acid versus intravenous zoledronic acid in treatment of bone metastases from breast cancer: a randomised, open label, non-inferiority phase 3 trial. Lancet Oncol 2014;15(1):114-122.

8. Amadori D, Aglietta M, Alessi B, et al. Efficacy and safety of weekly versus 4-weekly zoledronic acid for prolonged treatment of patients with bone metastases from breast cancer (ZOOM): a phase 3 , open-label, randomised, non-inferiority trial. Lancet Oncol 2013;14(7):663-670.

9. N. Hortobagyi. Efficacy and safety of continued zoledronic acid every 4 weeks versus every 12 weeks in women with bone metastases from breast cancer: Results of the OPTIMIZE-2 trial. J Clin Oncol 2014;32(15)

10. Early Breast Cancer Trialists' Collaborative Group (EBCTCG Adjuvant bisphosphonate treatment in early breast cancer: meta-analyses of individual patient data from randomized trials.). Lancet 2015;386:1353-1361

11. Gnant M, et al. Adjuvant denosumab in breast cancer (ABCSG-18): a multicentre, randomised, double-blind, placebo-controlled trial. Lancet 2015;386:433-443. 
12. Cummings $S$. Denosumab for prevention of fractures in postmenopausal women with osteoporosis. N Engl J Med 2009;361:756-765.

13. Gnant M. Role of bisphosphonates in postmenopausal women with breast cancer. Cancer Treat Rev 2014;40(3):476-84. doi: 10.1016/j. ctrv.2013.07.003.

14. Ben-Aharon I, Vidal L, Rizel S, et al. Bisphosphonates in the adjuvant setting of breast cancer therapy - effect on survival: a systematic review and meta-analysis. PLoS One 2013;8(8):e70044.

15. Lintermans A, Van Asten $\mathrm{K}$, Wildiers $\mathrm{H}$, et al. A prospective assessment of musculoskeletal toxicity and loss of grip strength in breast cancer patients receiving adjuvant aromatase inhibitors and tamoxifen, and relation with BMI. Breast Cancer Res Treat 2014;146(1):109-16.

\section{Breast cancer in young women}

1. Paluch-Shimon S, Pagani O, Partridge AH, et al. Second international consensus guidelines for breast cancer in young women (BCY2). Breast 2016;26:87-99.

2. Cardoso F, Loibl S, Pagani O, et al. The European Society of Breast Cancer Specialists recommendations for the management of young women with breast cancer. Eur J Cancer 2012;48(18):3355-3377.

3. Botteri E, Bagnardi V, Rotmensz N, et al. Analysis of local and regional recurrences in breast cancer after conservative surgery. Ann Oncol 2010;21(4):723-728

4. Vila J, Gandini S, Gentilini O. Overall survival according to type of surgery in young ( $\leq 40$ years) early breast cancer patients: A systematic meta-analysis comparing breast-conserving surgery versus mastectomy. Breast 2015;24(3):175-181.

5. Maishman T, Cutress RI, Hernandez A, et al. Local recurrence and breast oncological surgery in young women with breast cancer: The POSH Observational Cohort Study. Ann Surg 2016.

6. Frandsen J, Ly D, Cannon G, et al. In the Modern Treatment Era, Is Breast Conservation Equivalent to Mastectomy in Women Younger Than 40 Years of Age? A Multi-Institution Study. Int J Radiat Oncol Biol Phys 2015;93(5):1096-1103.

7. Antonini $\mathrm{N}$, Jones $\mathrm{H}$, Horiot $\mathrm{JC}$, et al. Effect of age and radiation dose on local control after breast conserving treatment: EORTC trial 22881 10882. Radiother Oncol 2007;82(3):265-271.

8. Poortmans PM, Collette L, Bartelink H, et al. The addition of a boost dose on the primary tumour bed after lumpectomy in breast conserving treatment for breast cancer. A summary of the results of EORTC 22881-10882 "boost versus no boost" trial. Cancer Radiother 2008;12(6-7):565-570.

9. Francis PA, Regan MM, Fleming GF, et al. Adjuvant ovarian suppression in premenopausal breast cancer. N Engl J Med 2015;372(5):436-446.

10. Bellet M, Gray KP, Francis PA, et al. Twelve-Month Estrogen Levels in Premenopausal Women With Hormone Receptor-Positive Breast Cancer Receiving Adjuvant Triptorelin Plus Exemestane or Tamoxifen in the Suppression of Ovarian Function Trial (SOFT): The SOFT-EST Substudy. $\mathrm{J}$ Clin Oncol 2016;34(14):1584-1593.

11. Lambertini M, Ceppi M, Poggio F, et al. Ovarian suppression using luteinizing hormone-releasing hormone agonists during chemotherapy to preserve ovarian function and fertility of breast cancer patients: a meta-analysis of randomized studies. Ann Oncol 2015;26(12):2408-2419.

12. Moore $\mathrm{HC}$, Unger JM, Phillips KA et al. Goserelin for ovarian protection during breast-cancer adjuvant chemotherapy. $\mathrm{N}$ Engl $\mathrm{J}$ Med 2015;372(10):923-932

13. Lambertini M, Boni L, Michelotti A, et al. Ovarian Suppression With Triptorelin During Adjuvant Breast Cancer Chemotherapy and Long-term Ovarian Function, Pregnancies, and Disease-Free Survival: A Randomized Clinical Trial. JAMA 2015;314(24):2632-2640.

14. Azim HA, Jr., Santoro L, Pavlidis N, et al. Safety of pregnancy following breast cancer diagnosis: a meta-analysis of 14 studies. Eur J Cancer 2011;47(1):74-83.

15. Azim HA, Jr., Kroman N, Paesmans $M$, et al. Prognostic impact of pregnancy after breast cancer according to estrogen receptor status: a multicenter retrospective study. J Clin Oncol 2013;31(1):73-79.

16. Lambertini M, Del Mastro L, Pescio MC, et al. Cancer and fertility preservation: international recommendations from an expert meeting. BMC Med 2016;14:1.

17. Mueller BA, Simon MS, Deapen D, Kamineni A, Malone KE, Daling JR. Childbearing and survival after breast carcinoma in young women. Cancer 2003;98(6):1131-1140.

18. Pagani $\mathrm{O}$, Ruggeri M, Manunta $\mathrm{S}$, et al. Pregnancy after breast cancer: Are young patients willing to participate in clinical studies? Breast 2015;24(3):201-207.

\section{Treatment in advanced age patients}

1. Gomez Portilla, Martinez de Lecea C, Cendoya I, Olabarria I, et al. Prevalence and treatment of oncologic disease in the elderly -an impeding challenge. Rev Esp Enferm Dig 2008;100:706-715.
2. Balducci L, Extermann M. Management of cancer in older person: a practical approach. Oncologist 2000;5:224-37.

3. Biganzoli L, Wildiers H, Oakman C, et al. Management of elderly patients with breast cancer: updated recommendations of the International Society of Geriatric Oncology (SIOG) and European Society of Breast Cancer Specialists (EUSOMA). Lancet Onc 2012;13:e148-e160.

4. Strulov SS, Hurria A, Muss $\mathrm{H}$. Breast cancer in women older than 80 years. ASCO. J Oncol Prac 2016;12(2):123-132.

5. Punglia RS, Hughes KS, Muss $H$. Management of older woman with early-stage breast cancer. ASCO Ed Book, 2015:48-55.

6. Sun J, Chia S. Adjuvant chemotherapy and HER2-directed therapy for early-stage breast cancer in the elderly. Br J Cancer 2016;22:1-6.

7. Hurria A, Togawa K, Mohile SG. Predicting chemotherapy toxicity in older adults with cancer: A prospective multicenter study. J Clin Oncol 2011;29(25):3457-345.

8. Mislang AR, Biganzoli L. Adjuvant systemic therapy in older breast cancer women. Can we optimize the level of care? Cancers (Basel) 2015;7:1191-1214.

9. Karuturi M, VanderWalde N, Muss $\mathrm{H}$. Approach and Management of Breast Cancer in the Elderly. Clin Geriatr Med 2016(32):133-153.

10. Elomrani F, Zine M, Afif M, et al. Management of early breast cancer in older women: from screening to treatment. Breast Cancer (Dove Med Press) 2015:7;165-171.

11. Kunkler IH, Williams LJ, Jack WJ, Cameron DA, Dixon JM; PRIME ॥ investigators. Breast-conserving surgery with or without irradiation in women aged 65 years or older with early breast cancer (PRIME II): a randomised controlled trial. Lancet Oncol 2015;16:266-273.

12. Tesarova P. Specific Aspects of Breast Cancer Therapy of Elderly Women. Biomed Res Int 2016;2016:1381695.

13. Matuschek C, Bölke E, Haussmann J, et al. The benefit of adjuvant radiotherapy after breast conserving surgery in older patients with low risk breast cancer- a meta-analysis of randomized trials. Radiat Oncol 2017:12(1):60. doi: 10.1186/s13014-017-0796-x.

14. Giugliano FM, Falivene S, Esposito E, External radiotherapy for breast cancer in the elderly. Aging Clin Exp Res 2017;29(Suppl 1):149-157. doi: 10.1007/s40520-016-0655-x. Epub 2016 Nov 11.

\section{Male breast cancer}

1. Registro Histopatológico de Neoplasias Malignas. Secretaría de Salud, México, 2008.

2. Giordano $\mathrm{SH}$. A review of the diagnosis and management of male breast cancer. Oncologist 2005;10:471-479.

3. Fentiman IS, Fourquet A, Hortobagyi GN. Male breast cancer. Lancet 2006;367:595-604.

4. Sousa B1, Moser E, Cardoso F. An update on male breast cancer and future directions for research and treatment. Eur J Pharmacol 2013;717(13):71-83. doi: 10.1016/j.ejphar.2013.03.037. Epub 2013 Mar 30.

5. Lanitis S, Rice AJ, Vaughan A, et al. Diagnosis and management of male breast cancer. World J Surg 2008;32:2471-2476.

\section{Breast cancer associated with pregnancy and breastfeeding}

1. Viswanathan S, Ramaswamy B. Pregnancy-associated breast cancer. Clin Obstet Gynecol 2011;54:546-555.

2. Litton JK, Theriault RL. Breast cancer and pregnancy: current concepts in diagnosis and treatment. Oncologist 2010;15(12):1238-1247.

3. Amant F, Loibl S, Neven P, Van Calsteren K. Breast cancer in pregnancy. Lancet 2012;379:570-579.

4. Amant $F$, Deckers $S$, Van Calsteren $\mathrm{K}$, et al. Breast cancer in pregnancy: Recommendations of an international consensus meeting. Eur $J$ Cancer 2010;46(18):3158-3168. doi: 10.1016/j.ejca.2010.09.010.

5. Amant F, Han SN, Gziri MM, Vandenbroucke T, Verheecke M, Van Calsteren K. Management of cancer in pregnancy. Best Pract Res Clin Obstet Gynaecol 2015;29(5):741-753. doi:10.1016/j.bpobgyn.2015.02.006.

6. Loibl S, Han SN, Amant F. Being pregnant and diagnosed with breast cancer. Breast Care 2012;7(3):204-209. doi: 10.1159/000339674.

7. Keleher A, Wendt R, Delpassand E, et al. The safety of lymphatic mapping in pregnant breast cancer patients using Tc-99m sulfur colloid. Breast J 2004:10(6):492-495.

8. Martin DD. Review of radiation therapy in the pregnant cancer patient. Clin Obstet Gynecol 2011;54:591-601.

9. Brewer M, Kueck A, Runowicz CD. Chemotherapy in pregnancy. Clin Obstet Gynecol 2011; 54:602-618.

10. van Hasselt JGC, van Calsteren K, Heyns L, et al. Optimizing anticancer drug treatment in pregnant cancer patients: pharmacokinetic analysis of gestation-induced changes for doxorubicin, epirubicin, docetaxel and paclitaxel. Ann Oncol 2014;25(10):2059-2065. doi: 10.1093/annonc/mdu140.

11. Cardonick E, Bhat A, Gilmandyar D, Somer R. Maternal and fetal outcomes of taxane chemotherapy in breast and ovarian cancer during pregnancy: case series and review of the literature. Ann Oncol 2012;23(12):3016-3023. doi: 10.1093/annonc/mds170. 
12. McGrath SE, Ring A. Chemotherapy for breast cancer in pregnancy: evidence and guidance for oncologists. Ther Adv Med Oncol 2011;3(2):7383. doi: $10.1177 / 1758834010392445$.

13. Azim F, Vandenbroucke $T$, Fumagalli $M$, et al. Pediatric Outcome after Maternal Cancer Diagnosed during Pregnancy. N Engl J Med 2015;373:1824-34.

14. Lambertini M, Peccatori FA, Azim HA. Targeted agents for cancer treatment during pregnancy. Cancer Treat Rev 2015;41(4):301-309. doi: 10.1016/j.ctrv.2015.03.001.

15. Pereg D, Koren G, Lishner M. Cancer in pregnancy: Gaps, challenges and solutions. Cancer Treat Rev 2008;34(4):302-312. doi: 10.1016/j. ctrv.2008.01.002

16. Azim HA, Santoro L, Russell-Edu W, Pentheroudakis G, Pavlidis N, Peccatori FA. Prognosis of pregnancy-associated breast cancer: A meta-analysis of 30 studies. Cancer Treat Rev 2012;38(7):834-842. doi: 10.1016/j.ctrv.2012.06.004

17. Amant F, von Minckwitz G, Han NS, Bontenbal M, et al. Prognosis of women with primary breast cancer diagnosed during pregnancy: results from an international collaborative study. J Clin Oncol 2013;31(20):25322539. doi: 10.1200/JCO.2012.45.6335.

\section{Hormone replacement therapy}

1. Fahlealn $\mathrm{M}$, Fornander $\mathrm{T}$, Johansson $\mathrm{H}$, et al. Hormone replace therapy after breast cancer: 10 year follow up of the Stockholm randomised trial. E J Cancer 2013;49:52-59

2. Women's Health Initiative Investigators. Risks and benefits of estrogen plus progestin in healthy postmenopausal women. Principal results from the Women's Health Initiative Randomized Controlled Trial. JAMA 2002;288:321-333.

3. Million Women Study Collaborators. Breast cancer and hormone-replacement therapy in the Million Women Study. Lancet 2003:362(9382):419-427.

4. Heiss G, Wallace R, Anderson GL, et al. for the WHI Investigators. Health risks and benefits 3 years after stopping randomized treatment with estrogen and progestin. JAMA 2008;299:1036-1045.

5. Sener SF, Winchester DJ, Winchester DP, et al. The effects of hormone replacement therapy on postmenopausal breast cancer biology and survival. Am J Surg 2009;197:403-407.

6. Reeves G, Beral V, Green J, et al. Hormonal therapy for menopause and breast-cancer risk by histological type: a cohort study and meta-analysis. Lancet Oncol 2006;7:910-918.

7. Antoine C, Liebens F, Carly B, Pastijn A, Neusy S, Rozenberg S. Safety of hormone therapy after breast cancer: a qualitative systematic review. Hum Reprod 2007;22(2):616-622.

8. von Schoultz E, Rutqvist LE. Menopausal hormone therapy after breast cancer: The Stockholm Randomized Trial. J Natl Cancer Inst 2005;97:533-535

9. Holmberg L, Anderson H. HABITS (hormonal replacement therapy after breast cancer-is it safe?), a randomized comparison: Trial stopped. Lancet 2004;363:453-455

10. Holmber $\mathrm{L}$, Iversen $\mathrm{O}$, et al. Increased risk of recurrence after hormone replacement therapy in breast cancer survivors. J Natl Cancer Inst 2008:100:475-482.

11. Kenemans P, Bundred NJ, Foidart JM et al. Safety and efficacy of tibolone in breast-cancer patients with vasomotor symptoms: a double-blind, randomised, non-inferiority trial (The livial Intervention Following Breast Cancer: Efficacy, Recurrence and Tolerability Endpoints, LIBERATE). Lancet Oncol 2009;10:135-146.

\section{Genetics and breast cancer}

1. Chávarri-Guerra $Y$, Villarreal-Garza $C$, Liedke PE, et al. Breast cance in Mexico: a growing challenge to health and the health system. Lancet Oncol 2012;13(8):e335-43.

2. Buys SS, Sandbach JF, Gammon A, Patel G, Kidd J, Brown KL, et al. A study of over 35,000 women with breast cancer tested with a 25-gene panel of hereditary cancer genes. Cancer 2017;123(10):1721-1730.

3. Ripperger T, Gadzicki D, Meindl A, Schlegelberger B. Breast cancer susceptibility: current knowledge and implications for genetic counseling. Eur J Hum Genet 2009;17:722-731.

4. Economopoulou P, Dimitriadis G, Psyrri A. Beyond BRCA: new hereditary breast cancer susceptibility genes. Cancer Treat Rev 2015;41:1-8.

5. Narod SA, Rodríguez AA. Genetic predisposition for breast cancer: BRCA1 and BRCA2 genes. Salud Publica Mex 2011;53:420-429.

6. Shannon KM, Chittenden A. Genetic testing by cancer site: breast. Cancer J 2012;18:310-319.

7. Vadaparampil ST, Scherr CL, Cragun D, et al. Pre-test genetic counseling services for hereditary breast and ovarian cancer delivered by non-genetics professionals in the state of Florida. Clin Genet 2015;85(5):473-477; doi: $10.1111 / \mathrm{cge} .12405$
8. Noar SM, Althouse BM, Ayers JW, et al. Cancer information seeking in the digital age: Effects of Angelina Jolie's prophylactic mastectomy announcement. Med Decis Making 2015;35:16-21.

9. Villarreal-Garza $C$, Alvarez-Gómez RM, Pérez-Plasencia C, Herrera LA, Herzog J, et al. Significant clinical impact of recurrent BRCA1 and BRCA2 mutations in Mexico. Cancer 2015;121:372-378.

10. Murray ML, Cerrato $F$, Bennett RL, Jarvik GP. Follow-up of carriers of BRCA1 and BRCA2 variants of unknown significance: variant reclassification and surgical decisions. Genet Med 2011;13:998-1005.

11. Shuen AY, Foulkes WD. Inherited mutations in breast cancer genes -risk and response. J Mammary Gland Biol Neoplasia 2011:16:3-15.

12. Villarreal-Garza C, Weitzel JN, Llacuachaqui M, Sifuentes E, Magallanes-Hoyos MC, et al. The prevalence of BRCA1 and BRCA2 mutations among young Mexican women with triple-negative breast cancer. Breast Cancer Res Treat. 2015;150:389-394.

13. Zugazagoitia J, Pérez-Segura $P$, Manzano A, et al. Limited family structure and triple-negative breast cancer (TNBC) subtype as predictors of BRCA mutations in a genetic counseling cohort of early-onset sporadic breast cancers. Breast Cancer Res Treat 2014;148:415-421.

14. The NCCN Clinical Practice Guidelines in Oncology Genetic/Familial High-Risk Assessment: Breast and ovarian V2.2017. National Comprehensive Cancer Network, 2016; http//www.nccn.org/.

15. Paluch-Shimon S, Cardoso F, Sessa C, Balmana J, Cardoso MJ, Gilbert F, Senkus E; ESMO Guidelines Committee. Prevention and screening in BRCA mutation carriers and other breast/ovarian hereditary cancer syndromes: ESMO Clinical Practice Guidelines for cancer prevention and screening. Ann Oncol 2016;27(suppl 5):v103-v110

16. Laduca $H$, Stuenkel AJ, Dolinsky JS, et al. Utilization of multigene panels in hereditary cancer predisposition testing: analysis of more than 2,000 patients. Genet Med 2014;16:830-837.

\section{Psycho-oncologic aspects of breast cancer}

1. Alvarado SA. El psicólogo y su contribución en la Oncología. Gaceta Mexicana de Oncología 2008;7(2):27.

2. Cano A. Control emocional, estilo represivo de afrontamiento y cáncer: ansiedad y cáncer. Psicooncología 2005;2:71-80.

3. Bultz BD, Carlson L. Emotional distress: The sixth vital sign: future directions in cancer care. Psychooncol 2006;15:93-5.

4. Robles R, Morales M, Jiménez LM, Morales J. Depresión y ansiedad en mujeres con cáncer de mama: el papel de la afectividad y el soporte social. Psicooncología. 2009;6(1):191-201.

5. Campbell-Enns HJ, et al. The psychosocial experiences of women with breast cancer across the lifespan: a systematic review. Psychooncology 2016 Sep 20. doi: 10.1002/pon.4281.

6. Brandao T, Schulz MS, Matos PM. Psychological adjustment after breast cancer: A systematic Review of longitudinal studies. Psychooncology 2016 Jul. 20. doi: 10.1002/pon.4230.

7. Choi EK, Kim IR, Chang O, Kang D, Nam SJ, Lee JE, Lee SK, Im YH, Park $\mathrm{YH}$, Yang JH, Cho J. Impact of chemotherapy-induced alopecia distress on body image, psychosocial well-being, and depression in breast cancer patients. Psychooncology 2014; 23:1103-1110.

8. Holland JC, Breitbart WS, Jacobsen PB, Lederberg MS. Psycho-Oncology. New York: Oxford, 2010.

9. Cardoso F, et al. The European Society of Breast Cancer Specialists recommendations for the management of young women with breast cancer. Eur J Cancer. 2012;18:3355-3377.

10. Champion $\mathrm{L}$, et al. Comparison of younger and older breast cancer survivors and age-matched controls on specific and overall quality of life domains. Cancer 2014;120:2237-2246.

11. Vázquez OG, Castillo ER, Huertas LA, García AM, Ponce JUA, Manzanilla EO, Aguilar SA. Guía de práctica clínica para la atención psico-oncológica del cuidador primario informal de pacientes con cáncer. Psicooncología 2015;12(1):87-104.

12. Manne S, Kashy D, Siegel S, Myers S, Heckman C, Ryan D. Unsupportive partner behaviors, social-cognitive and psychological outcomes in couple coping with early stage breast cancer. J Fam Psychol 2014; 28(2):214-224.

13. Kohli S, Griggs JJ, Roscoe JA, et al. Self-reported cognitive impairment in patients with cancer. J Oncol Pract 2007;3:54-59.

14. Cohen M, Mabjish AA, Zidan J. Comparison of Arab breast cancer survivors and healthy controls for spousal relationship, body image, and emotional distress. QualLife Res 2011;20:191-198.

15. Almanza-Muñoz JJ, Juárez IR, Pérez S. Traducción, adaptación y validación del Termómetro de Distrés en una muestra de pacientes mexicanos con cáncer, 2008

16. Galindo O, Benjet C, Juárez F, Rojas E, Riveros A, Aguilar-Ponce JL, Álvarez MA, Alvarado S. Propiedades psicométricas de la Escala Hospitalaria de Ansiedad y Depresión (HADS) en una población de pacientes oncológicos mexicanos. Salud Ment 2015;38(4). 
17. Galindo-Vazquez O, Benjet C, Cruz-Nieto MH, Rojas-Castillo E, Riveros-Rosas A, Meneses-García A, Aguilar-Ponce JL, Álvarez-Avitia MA, Alvarado-Aguilar S. Psychometric properties of the Zarit Burden Interview in Mexican caregivers of cancer patients. Psychooncology 2015;24(5):612-615.

18. Matthews H, Grunfeld EA, Turner A. The efficacy of interventions to improve psychosocial outcomes following surgical treatment for breast cáncer: A Systematic review and meta-analysis. Psychooncology 2016 Jun 22. doi: 10.1002/pon.4199

19. Moral DR. Estudio de validación de la Escala de Ajuste Diádico (DAS) en población mexicana. Revista Internacional de Ciencias Sociales y Humanidades 2009;19(1):113-138

20. Font $\mathrm{A}$, Rodríguez E. Eficacia de las intervenciones psicológicas en mujeres con cáncer de mama. Psicooncología 2007:4(2):423-446.

21. Galindo-Vázquez O, Pérez-Barrientos H, Alvarado-Aguilar S, et al Efectos de la terapia cognitivo conductual en el paciente oncológico: una revisión. Rev Gamo 2013;12(2):108-115.

XXIV. Physical rehabilitation for the breast cancer patient

1. 2003 consensus of the International Society of Lymphology. The diag nosis and treatment of peripheral lymphedema Executive Committee. www.u.arizona.edu/\%7Ewitte/ISL.htm. Consulta el 19 de abril de 2011.

2. Rockson SG. Diagnosis and management of lymphatic vascular disease. J Am Coll Cardiol 2008;52(10):799-806.

3. Graham PH. Compression prophylaxis may increase the potential for flight-associated lymphoedema after breast cancer treatment. Breast 2002;11:66.

4. Merchant SJ, Chen SL. Prevention and management of lymphedema after breast cancer treatment. Breast J 2015;21(3):276-284.

5. Foldi E, Foldi $M$, Weissleder $\mathrm{H}$. Conservative treatment of lymphoedema of the limbs. Angiology 1985;36(3):171-180.

6. Asdourian MS, Skolny MN, Brunelle C, Seward CE, Salama L, Taghian AG. Precautions for breast cancer-related lymphoedema: risk from air travel, ipsilateral arm blood pressure measurements, skin puncture, extreme temperatures, and cellulitis. Lancet Oncol 2016:17(9);e392-e405.

7. Morris $\mathrm{C}$, Wonders KY. Concise review on the safety of exercise on symptoms of lymphedema. World J Clin Oncol 2015;6(4):43-44.

8. Herdman TH, Shigemi K, MANDA International. Diagnósticos enfermeros. Definiciones y clasificación 2015-2017. Barcelona: Elsevier, 2015.

XXV. Palliative care in metastatic breast cancer

1. Hui D, Bruera D. Integrating palliative care into the trajectory of cancer care. Nat Rev Clin Oncol. 2016;13(3):159-171. doi:10.1038/nrclinonc.2015.201.

2. Sepulveda CM, Yoshida A, Ullrich T: Palliative care: The World Health organization's global perspective. J Pain Symptom Manage 2002;24:91-96.

3. Hui D, Bruera D. Models of integration of oncology and palliative care Ann Palliat Med 2015;4(3):89-98.

4. Smith TJ, Temin S, Alesi ER, Abernethy AP, et al. American Society of Clinical Oncology provisional clinical opinion: the integration of palliative care in to standard oncology care. J Clin Oncol 2012;30:880-887.

5. Levy M, Smith T, Alvarez-Perez A, Back A, Baker JN, Beck AC, Block S, et al. Palliative Care Version 1.2016. J Natl Compr Canc Netw 2016;14(1):82-113.

6. Inouye SK, van Dyck CH, Alessi CA, Balkin S, Siegal AP, Horwitz RI. Clarifying confusion: the confusion assessment method. A new method for detection of delirium. Ann Intern Med. 1990;113(12):941-948.

7. Clinical Practice Guidelines in Oncology (NCCN Guidelines). Breast Cancer Version 3.2015. Breast Cancer update. Disponible en: http://www. consensocancermamario.com/guias/NCCN_2015.pdf. Accesado el 16 de enero 2017.

8. Hui D, Mori M, Watanabe SH, Caraceni A, Strasser F, Saarto T, et al. Referral criteria for outpatient specialty palliative cancer care: an international consensus. Lancet Oncol 2016;16:e552-e559.

\section{PARTICIPANTS IN THE CONSENSUS SEVENTH REVISION}

\section{Coordinators}

Jesús Cárdenas Sánchez, MD

Medical oncologist

Instituto Estatal de Cancerología, SS

Colima, Col.
Juan Enrique Bargalló Rocha, MD

Surgical oncologist

Instituto Nacional de Cancerología, SS

Ciudad de México

Verónica Bautista Piña, MD

Pathologist

Instituto de Enfermedades de la Mama, FUCAM

Ciudad de México

Guadalupe Cervantes Sánchez, MD

Medical oncologist

Centro Médico Nacional 20 de Noviembre, ISSSTE

Ciudad de México

Aura A. Erazo Valle-Solís, MD

Medical oncologist

Centro Médico Nacional 20 de Noviembre, ISSSTE

Ciudad de México

Christian Haydeé Flores Balcázar, MD

Radiation oncologist

Instituto Nacional de Ciencias Médicas y Nutrición

Salvador Zubirán, SS

Ciudad de México

Antonio Maffuz Aziz, MD

Surgical oncologist

Instituto de Enfermedades de la Mama, FUCAM

Ciudad de México

Víctor Manuel Pérez Sánchez, MD

Pathologist

Instituto Nacional de Cancerología, SS

Ciudad de México

Adela Poitevin Chacón, MD

Radiation oncologist

Médica Sur

Ciudad de México

Efraín Salas González, MD

Medical oncologist

Centro Médico de Occidente, IMSS

Guadalajara, Jal.

Laura Torrecillas Torres, MD

Medical oncologist

Centro Médico Nacional 20 de Noviembre, ISSSTE

Ciudad de México

Vicente Valero Castillo, MD

Medical oncologist

M. D. Anderson Cancer Center

Houston, TX, United States

Participants

Aldo Antonio Alcaraz Wong, MD

Pathologist

Centro Médico de Occidente, IMSS

Guadalajara, Jal. 
Fernando Aldaco Sarvide, MD

Medical oncologist

Centro Médico Nacional 20 de Noviembre, ISSSTE

Ciudad de México

Silvia Allende Pérez, MD

Palliativist

Instituto Nacional de Cancerología, SS

Ciudad de México

Isabelle Aloi-Timeus Salvato, BPT

Physiotherapist

Hospital ABC

Ciudad de México

Salvador Alvarado Aguilar, BS

Psycho-oncologist

Instituto Nacional de Cancerología, SS

Ciudad de México

Isabel Alvarado Cabrero, MD

Pathologist

Hospital de Oncología, CMN Siglo XXI, IMSS

Ciudad de México

Alberto Alvarado Miranda, MD

Medical oncologist

Instituto Nacional de Cancerología, SS

Ciudad de México

Adriana Alvarado Zermeño, MD

Radiation oncologist

Centro Médico de Occidente, IMSS

Guadalajara, Jal.

Rosa María Álvarez Gómez, MD

Geneticist

Instituto Nacional de Cancerología, SS

Ciudad de México

Claudia Arce Salinas, MD

Medical oncologist

Instituto Nacional de Cancerología, SS

Ciudad de México

Sinuhé Barroso Bravo, MD

Surgical oncologist

Hospital de Oncología, CMN Siglo XXI, IMSS

Ciudad de México

Nimbe Barroso Quiroga, MD

Radiation oncologist

Clínica de Radioterapia de Occidente

Guadalajara, Jal.

María Yisel Bautista Hernández, MD

Radiation oncologist

Hospital General de México, SS

Ciudad de México

Verónica Bautista Piña, MD

Pathologist
Instituto de Enfermedades de la Mama, FUCAM

Ciudad de México

Marissa Bravo Cañón, MD

Radiologist

Grupo CT Scanner de México

Puebla, Pue.

Paula Anel Cabrera Galeana, MD

Medical oncologist

Instituto Nacional de Cancerología, SS

Ciudad de México

Verónica Cedillo Compeán, BPT

Physiotherapist

Instituto Nacional de Cancerología, SS

Ciudad de México

Yanín Chavarri Guerra, MD

Medical oncologist

Instituto Nacional de Ciencias Médicas y Nutrición

Salvador Zubirán, SS

Ciudad de México

Mariana Chávez MacGregor, MD

Medical oncologist

M. D. Anderson Hospital

Houston, TX, United States

Jessica Chávez Nogueda, MD

Radiation oncologist

Hospital de Oncología, CMN Siglo XXI, IMSS

Ciudad de México

Jaime Corona Rivera, MD

Surgical oncologist

Hospital Country 2000

Guadalajara, Jal.

Carlos A. Domínguez Reyes, MD

Surgical oncologist

Instituto de Enfermedades de la Mama, FUCAM

Ciudad de México

Mónica Drucker Zertuche, MD

Reconstructive plastic surgeon

Instituto Nacional de Cancerología, SS

Ciudad de México

Mario Escobar Gómez, MD

Medical oncologist

Hospital General de México, SS

Ciudad de México

Nereida Esparza Arias, MD

Surgical oncologist

Instituto Nacional de Cancerología, SS

Ciudad de México

Miguel Ángel Farías Alarcón, MD

Surgical oncologist

Centro Médico Nacional 20 de Noviembre, ISSSTE 
Ciudad de México

Jimena Figueroa Valero, BPT

Physiotherapist

Universidad Anáhuac Norte

Ciudad de México

Jesús Manuel Flores Castro, MD

Radio-oncologist

Instituto Nacional de Cancerología, SS

Ciudad de México

Sonia María Flores Moreno, MD

Surgical oncologist

Doctors Hospital

Monterrey, N. L.

Óscar Galindo Vázquez, Psy.D

Instituto Nacional de Cancerología, SS

Ciudad de México

Georgina Garnica Jaliffe, MD

Medical oncologist

Hospital General de México Eduardo Liceaga, SS

Ciudad de México

Gabriela Sofía Gómez Macías, MD

Pathologist

Centro de Cáncer de Mama, Hospital San José

Monterrey, N. L.

Daniela Gómez Pue, MD

Gynecologic oncologist

Hospital ABC

Ciudad de México

Manuel Ismael González Geronis, MD

Surgeon oncologist

Clínica 25, IMSS

Monterrey N. L.

Juan Francisco González Guerrero, MD

Medical oncologist and radiotherapist

Centro Universitario contra el Cáncer, UANL

Monterrey, N. L.

José Luis González Vela, MD

Medical oncologist

Clínica Regional, ISSSTE

Monterrey, N. L.

Rocío Grajales Álvarez, MD

Medical oncologist

Hospital de Oncología, CMN Siglo XXI,

IMSS Ciudad de México

Mercedes Hernández González, MD

Pathologist

Hospital General de México, SS

Ciudad de México

Luz del Carmen Hernández Hernández, MS

Epidemiologist
Centro Nacional de Equidad de Género y Salud Reproductiva, SS

Ciudad de México

José Hinojosa Gómez, MD

Radiation oncologist

Instituto Nacional de Cancerología, SS

Ciudad de México

Joel Jiménez Alatorre, MD

Surgical oncologist

Instituto Jalisciense de Cancerología, SS

Guadalajara, Jal.

Fernando U. Lara Medina, MD

Medical oncologist

Instituto Nacional de Cancerología, SS

Ciudad de México

María del Carmen Lara Tamburrino, MD

Radiologist

C. T. Scanner

Ciudad de México

Ana Lluch Hernández, MD

Medical oncologist

Hospital Clínico

Valencia, Spain

Ignacio Lugo Beltrán, MD

Reconstructive plastic surgeon

Centro Médico Nacional 20 de Noviembre, ISSSTE

Ciudad de México

Miguel Machado Reyes, MD

Gynecologic oncologist

Centro Oncológico ISSEMYM

Toluca, Estado de México

Alejandro Maciel Miranda, MD

Reconstructive plastic surgeon

Instituto Nacional de Cancerología, SS

Ciudad de México

Fernando Mainero Ratchelous, MD

Surgical oncologist

Hospital de Ginecoobstetricia No.4, IMSS

Ciudad de México

Dolores de la Mata Moya, MD

Radiation oncologist Hospital ABC

Ciudad de México

Heriberto Medina Franco, MD

Surgical oncologist

Instituto Nacional de la Nutrición, SS

Ciudad de México

Alejandro Mohar Betancourt, MD

Epidemiologist and pathologist

Instituto Nacional de Cancerología, SS

Ciudad de México 
Edith Monreal Carrillo, MD

Palliativist

Instituto Nacional de Cancerología

Ciudad de México

Flavia Morales Vázquez, MD

Medical oncologist

Instituto de Enfermedades de la Mama, FUCAM

Ciudad de México

Aída Mota García, MD

Radiation oncologist

Instituto Nacional de Cancerología, SS

Ciudad de México

María Paulina Núñez Martínez, MD

Geneticist

Instituto Nacional de Cancerología, SS

Ciudad de México

Guillermo Olivares Beltrán, MD

Medical oncologist

Hospital ABC Ciudad de México

Martha Orozco Quiyono, MD

Geneticist

Centro Médico Nacional 20 de Noviembre, ISSSTE

Ciudad de México

Cecilia Ortiz de Iturbide, MD

Radiologist

Hospital Ángeles del Pedregal

Ciudad de México

Cecilia Magdalena Pavón Hernández, MD

Radiologist

Instituto Nacional de Cancerología, SS

Ciudad de México

Perla Pérez Pérez, MD

Medical oncologist

Centro Médico Nacional 20 de Noviembre, ISSSTE

Ciudad de México

Claudia Pineda Flores, Psy.D

Facultad de Psicología, UNAM

Ciudad de México

Gregorio Quintero Beuló, MD

Surgical oncologist

Hospital General de México, SS

Ciudad de México

María Teresa Ramírez Ugalde, MD

Surgical oncologist

Instituto Nacional de Cancerología, SS

Ciudad de México

Ma. Eugenia Ramos Rayón, BPT

Physiotherapist

Hospital de Oncología, CMN Siglo XXI, IMSS

Ciudad de México
Samuel Rivera Rivera, MD

Surgical oncologist

Hospital de Oncología, CMN Siglo XXI, IMSS

Ciudad de México

Carlos D. Robles Vidal, MD

Surgical oncologist

Instituto Nacional de Cancerología, SS

Ciudad de México

Sergio Rodríguez Cuevas, MD

Surgical oncologist

Instituto de Enfermedades de la Mama, FUCAM

Ciudad de México

Amelia Rodríguez Trejo, MD

Gynecologic oncologist

Centro Estatal de Oncología, SS Tepic, Nay.

Edith Rojas Castillo, Psy.M

Instituto Nacional de Cancerología, SS

Ciudad de México

Julia Sáenz Frías, MD

Radiation oncologist Clínica 25, IMSS

Monterrey, N. L.

Francisco Miguel Said Lemus, MD

Reconstructive plastic surgeon

Instituto de Enfermedades de la Mama, FUCAM

Ciudad de México

Benito Sánchez Llamas, MD

Medical Oncologist

Centro Médico de Occidente, IMSS

Guadalajara, Jal.

Erik Santamaría Linares, MD

Reconstructive plastic surgeon

Hospital Manuel Gea González, SS

Ciudad de México

Bernardino Gabriel Santiago Concha, MD

Radiation oncologist

Instituto Nacional de Cancerología, SS

Ciudad de México

Héctor Santiago Payán, MD

Pathologist Hospital Santa Fe

Ciudad de México

Rodrigo Serrano Ortiz, MD

Surgical oncologist

Centro Oncológico Estatal, ISSEMYM

Toluca, Estado de México

Robin Jennifer Shaw Dulin, MD

Gynecologic oncologist

Instituto Nacional de Cancerología, SS

Ciudad de México

Juan Alejandro Silva, MD

Medical oncologist 
Hospital de Oncología, CMN Siglo XXI, IMSS

Ciudad de México

Gerónimo Tavares Macías, MD

Pathologist

Centro Médico de Occidente, IMSS

Guadalajara, Jal.

Dr. Rafael Vázquez Romo

Surgical oncologist

Instituto Nacional de Cancerología, SS

Ciudad de México

Arturo Vega Saldaña, MD

Gynecologist

C. N. de Equidad y Género y Salud Reproductiva, SS

Ciudad de México

Graciela Velázquez Delgado, MD

Pathologist

Centro Estatal de Atención Oncológica, SS

Morelia, Mich.

Emma Verástegui Avilés, MD

Palliativist

Instituto Nacional de Cancerología, SS

Ciudad de México
Silvia Vidal Millán, MD

Geneticist

Instituto Nacional de Cancerología, SS

Ciudad de México

Patricia Villarreal Colín, MD

Gynecologic oncologist

Instituto Nacional de Cancerología, SS

Ciudad de México

Cynthia Villarreal Garza, MD

Medical oncologist

Centro de Cáncer de Mama, TEC de Monterrey/

Instituto Nacional de Cancerología, SS

Monterrey N. L./

Ciudad de México

Yolanda Villaseñor Navarro, MD

Radiologist

Instituto Nacional de Cancerología, SS

Ciudad de México

Michelle Aline Villavicencio Queijeiro, MD

Radiation oncologist

Centro Médico Nacional 20 de Noviembre, ISSSTE

Ciudad de México 